\title{
The Bandwidth Theorem in Sparse Graphs
}

\author{
Peter Allen \\ Julia Böttcher \\ Julia Ehrenmüller \\ Anusch Taraz
}

Received 21 February 2019; Published 15 May 2020

\begin{abstract}
The bandwidth theorem [Mathematische Annalen, 343(1):175-205, 2009] states that any $n$-vertex graph $G$ with minimum degree $\left(\frac{k-1}{k}+o(1)\right) n$ contains all $n$-vertex $k$ colourable graphs $H$ with bounded maximum degree and bandwidth $o(n)$. We provide sparse analogues of this statement in random graphs as well as pseudorandom graphs.

More precisely, we show that for $p \gg\left(\frac{\log n}{n}\right)^{1 / \Delta}$ asymptotically almost surely each spanning subgraph $G$ of $G(n, p)$ with minimum degree $\left(\frac{k-1}{k}+o(1)\right) p n$ contains all $n$-vertex $k$-colourable graphs $H$ with maximum degree $\Delta$, bandwidth $o(n)$, and at least $C p^{-2}$ vertices not contained in any triangle. A similar result is shown for sufficiently bijumbled graphs, which, to the best of our knowledge, is the first resilience result in pseudorandom graphs for a rich class of spanning subgraphs. Finally, we provide improved results for $H$ with small degeneracy, which in particular imply a resilience result in $G(n, p)$ with respect to the containment of spanning bounded degree trees for $p \gg\left(\frac{\log n}{n}\right)^{1 / 3}$.
\end{abstract}

\section{Introduction}

A central topic in extremal graph theory is to determine minimum degree conditions which force a graph $G$ to contain a copy of some large or even spanning subgraph $H$. The prototypical example of such a theorem is Dirac's theorem [21], which states that if $\delta(G) \geq \frac{1}{2} v(G)$ and $v(G) \geq 3$, then $G$ is Hamiltonian. Analogous results were established for a wide range of spanning subgraphs $H$ with bounded maximum degree such as powers of Hamilton cycles, trees, or $F$-factors for any fixed graph $F$ (see e.g. [32] for a survey). One feature that all these subgraphs $H$ have in common is that their bandwidth is small. The bandwidth of a graph $H$ is the minimum $b$ such that there is a labelling of the vertex set of $H$ by integers $1, \ldots, n$ with $|i-j| \leq b$ for every edge $i j$ of $H$. And indeed, it was shown in [12] that a more general result holds, which provides a minimum degree condition forcing any spanning bounded degree subgraphs of small bandwidth. 
Theorem 1 (Bandwidth Theorem [12]). For every $\gamma>0, \Delta \geq 2$, and $k \geq 1$, there exist $\beta>0$ and $n_{0} \geq 1$ such that for every $n \geq n_{0}$ the following holds. If $G$ is a graph on $n$ vertices with minimum degree $\delta(G) \geq\left(\frac{k-1}{k}+\gamma\right) n$ and if $H$ is a $k$-colourable graph on $n$ vertices with maximum degree $\Delta(H) \leq \Delta$ and bandwidth at most $\beta n$, then $G$ contains a copy of $H$.

We remark that in contrast to the above mentioned earlier results for specific bounded degree spanning subgraphs the minimum degree condition in this theorem has an error term $\gamma n$, and it is known that this cannot completely be omitted in this general statement. In that sense the minimum degree condition in Theorem 1 is best-possible. It is also known that the bandwidth condition cannot be dropped completely (see [12] for further explanations). Moreover, this condition does not limit the class of graphs under consideration unreasonably, because many interesting classes of graphs have sublinear bandwidth. Indeed, it was shown in [11] that for bounded degree $n$-vertex graphs, restricting the bandwidth to $o(n)$ is equivalent to restricting the treewidth to $o(n)$ or forbidding linear sized expanding subgraphs, which implies that bounded degree planar graphs, or more generally classes of bounded degree graphs defined by forbidding some fixed minor have bandwidth $o(n)$. Generalisations of Theorem 1 were obtained in $[9,13,27,33]$.

In this paper we are interested in the transference of Theorem 1 to sparse graphs. Such transference results recently received much attention, including for example the breakthrough result on the transference of Turán's theorem to random graphs by Conlon and Gowers [14] and Schacht [39]. The random graph model we shall consider here is the binomial random graph $G(n, p)$, which has $n$ vertices, and each pair of vertices forms an edge independently with probability $p$. We shall study the asymptotic appearance of spanning subgraphs $H_{n}$ in $G(n, p)$ under adversarial edge deletions. We denote the sequence of graphs we consider by $H=\left(H_{n}\right)$, and, abusing notation slightly also often write $H$ for the graph $H_{n}$, when it is clear from the context what $n$ is.

The appearance of large or spanning subgraphs of $G(n, p)$ was studied since the early days of probabilistic combinatorics and by now many important results were obtained. Gems include the theorem of Riordan [38] which gives a very good, and in many cases tight, upper bound on the threshold for $G(n, p)$ to contain a general sequence of spanning graphs $H=\left(H_{n}\right)$, and the Johansson-Kahn-Vu theorem [26] on $F$-factors, which we state for the case $F=K_{k}$.

Theorem 2 (Johansson, Kahn and $\mathrm{Vu}[26])$. For each $k \geq 3$ there exists $C>0$ such that the following

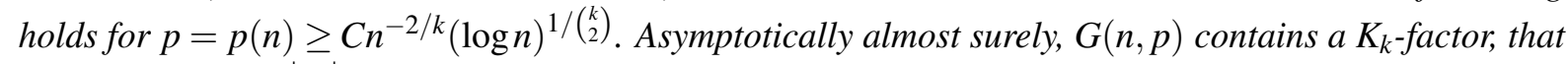
is, a collection of $\left\lfloor\frac{n}{k}\right\rfloor$ pairwise vertex-disjoint copies of $K_{k}$.

For a sequence of spanning graphs $H=\left(H_{n}\right)$ with maximum degree $\Delta(H) \leq \Delta$, Riordan's theorem implies that $G(n, p)$ asymptotically almost surely (a.a.s.), that is, with probability tending to 1 as $n$ tends to infinity, contains $H$ as a subgraph if $p \cdot n^{\frac{2}{\Delta+1}-\frac{2}{\Delta(\Delta+1)}} \rightarrow \infty$. This is not believed to be best possible.

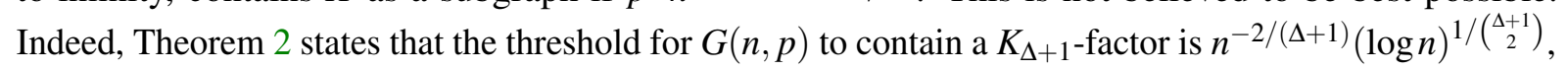
and it is conjectured in [23] that above this probability we also get any other sequence of spanning graphs $H=\left(H_{n}\right)$ with $\Delta(H) \leq \Delta$. This was proved, using Theorem 2, to be true for almost spanning graphs by Ferber, Luh, and Nguyen [23]. 
The BANDWIDTh TheOREM IN SPARSE GRAPHS

Theorem 3 (Ferber, Luh, and Nguyen [23]). For every $\varepsilon>0$ and $\Delta \geq 1$, and every sequence $H=$ $\left(H_{n}\right)$ of graphs with $v(H) \leq(1-\varepsilon) n$ and $\Delta(H) \leq \Delta$, the random graph $G(n, p)$ a.a.s. contains $H$ if $p \cdot n^{2 /(\Delta+1)} /(\log n)^{1 /\left(\begin{array}{c}\Delta+1 \\ 2\end{array}\right)} \rightarrow \infty$.

Better bounds are available if we further know that the degeneracy of $H$ is bounded by a constant much smaller than $\Delta(H)$. The degeneracy of $H$ is the smallest integer $D$ such that any subgraph of $H$ has a vertex of degree at most $D$. Surprisingly, for this class of graphs $H$ already Riordan's theorem implies an essentially optimal bound.

Corollary 4 (of Riordan's theorem [38]). For every $\Delta \geq 1$ and $D \geq 3$, and every sequence $H=\left(H_{n}\right)$ of graphs with $v(H) \leq n$ and $\Delta(H) \leq \Delta$ and degeneracy at most $D$, the random graph $G(n, p)$ a.a.s. contains $H$ if $p \cdot n^{1 / D} \rightarrow \infty$.

This is best possible because a simple first moment calculation shows that if $p \cdot n^{1 / D} \rightarrow 0$ then $G(n, p)$ a.a.s. does not contain the $D$-th power of a Hamilton path, which is a $D$-degenerate graph with maximum degree $2 D$.

Two features that both Riordan's theorem and Theorem 2 (and consequently all results which rely on them, such as Theorem 3 and Corollary 4) have in common is that their proofs are non-constructive, and the proof techniques do not allow for so-called universality results. A graph $G$ is said to be universal for a family $\mathcal{H}$ of graphs if $G$ contains copies of all graphs in $\mathcal{H}$ simultaneously. The random graph $G(n, p)$ is known to be universal for various families of graphs, but in almost all cases we only know an upper bound on the threshold for universality, which we do not believe is the correct answer.

The reason why probabilistic existence results such as Corollary 4 do not imply universality is that in $G(n, p)$ the failure probability for containing any given spanning graph $H$ without isolated vertices is at least $(1-p)^{n-1}$, the probability that a fixed vertex of $G(n, p)$ is isolated. This probability is too large to apply a union bound. Thus, to prove universality results, one needs to show that any graph $G$ with some collection of properties that $G(n, p)$ a.a.s. possesses must contain any given $H \in \mathcal{H}$. Using this approach, and improving on a series of earlier results, Dellamonica, Kohayakawa, Rödl and Ruciński [20] obtained the following universality result for the family $\mathcal{H}(n, \Delta)$ of $n$-vertex graphs with maximum degree $\Delta$.

Theorem 5 (Dellamonica, Kohayakawa, Rödl and Ruciński [20]). For all $\Delta \geq 3$ there is $C$ such that if $p \geq C\left(\frac{\log n}{n}\right)^{1 / \Delta}$ then $G(n, p)$ is a.a.s. universal for $\mathcal{H}(n, \Delta)$.

However, it is conjectured that universality and the appearance of a $K_{\Delta+1}$-factor occur together, at the threshold given in Theorem 3. A probability bound which is better, but still far from the conjectured truth, was so far only established for almost spanning graphs by Conlon, Ferber, Nenadov and Škorić [17], who showed that for $\Delta \geq 3$, if $p \gg n^{-1 /(\Delta-1)} \log ^{5} n$ then $G(n, p)$ is a.a.s. universal for $\left.\mathcal{H}((1-o(1)) n, \Delta)\right)$. For graphs with small degeneracy, again, the following better bound exists, but this also is far away from the threshold in Corollary 4, which is a plausible candidate for the correct answer.

Theorem 6 (Allen, Böttcher, Hàn, Kohayakawa, Person [2]). For all $\Delta, D \geq 1$ there is $C$ such that if $p \geq C\left(\frac{\log n}{n}\right)^{1 /(2 D+1)}$ then $G(n, p)$ is a.a.s. universal for all graphs in $\mathcal{H}(n, \Delta)$ with degeneracy at most $D$.

Very recently Conlon and Nenadov [16] established an essentially best possible bound on $p$ for an almost spanning analogue: They showed that $G(n, p)$ is a.a.s. universal for all graphs in $\mathcal{H}((1-\varepsilon) n, \Delta)$ with degeneracy at most $D$ for $p \geq\left(\frac{C \log ^{2} n}{n \log \log n}\right)^{1 / D}$. 
Furthermore, one may ask how robustly $G(n, p)$ contains certain large subgraphs $H$. Questions of this type were considered by Alon, Capalbo, Kohayakawa, Rödl, Ruciński and Szemerédi [5], and further popularised by Sudakov and $\mathrm{Vu}$ [41], who introduced the term local resilience. Let $\mathcal{P}$ be a monotone increasing graph property and let $G$ be a graph in $\mathcal{P}$. The local resilience of $G$ with respect to $\mathcal{P}$ is defined to be the minimum $r \in \mathbb{R}$ such that by deleting at each vertex $v \in V(G)$ at most $r \operatorname{deg}(v)$ edges one can obtain a graph not in $\mathcal{P}$. In this language, for example, Theorem 1 says that the local resilience of $K_{n}=G(n, 1)$ with respect to being universal for all $k$-colourable graphs in $\mathcal{H}(n, \Delta)$ with sublinear bandwidth is $\frac{1}{k}-o(1)$, and a sparse analogue asks for a similar local resilience result to hold a.a.s. for $G(n, p)$.

Lee and Sudakov [34] obtained a very strong local resilience result for Hamilton cycles. Improving on [41], they showed that the local resilience of $G(n, p)$ with respect to Hamiltonicity is a.a.s. at least $\frac{1}{2}-o(1)$ when $p=\Omega\left(\frac{\log n}{n}\right)$. This is optimal up to the constant factor, as below this probability $G(n, p)$ is itself a.a.s. not Hamiltonian. Triangle factors were investigated by Balogh, Lee and Samotij [7], who proved that the local resilience of $G(n, p)$ with respect to the containment of a triangle factor on $n-O\left(p^{-2}\right)$ vertices is a.a.s. $\frac{1}{3}-o(1)$ if $p \gg\left(\frac{\log n}{n}\right)^{1 / 2}$. It was observed by Huang, Lee, and Sudakov [24] that we cannot hope to cover all vertices in the host graph with triangles: Already for constant $p$ it is easy to delete all edges in the neighbourhood of $\Theta\left(p^{-2}\right)$ vertices in $G(n, p)$ without violating the resilience condition. Very recently Noever and Steger [37] showed that the local resilience of $G(n, p)$ with respect to containing a $(1-o(1)) n$-vertex squared cycle (a cycle with all edges between vertices at distance 2 added) is a.a.s. $\frac{1}{3}-o(1)$ provided $p \gg n^{-1 / 2+o(1)}$. Even more recently, Nenadov and Škorić [36] removed the log-factor from the probability bound of [7]. These results are notable in that the bounds on $p$ are close to optimal: for $p \ll n^{-1 / 2}$, a.a.s. most edges of $G(n, p)$ are not in triangles and one can obtain a triangle-free graph by deleting only a tiny fraction of edges at each vertex, so that the local resilience of $G(n, p)$ with respect to containing triangles is $o(1)$. Furthermore, for $p \ll n^{-1 / 2}$ the random graph $G(n, p)$ itself does not contain any $(1-o(1)) n$-vertex squared cycle.

More general subgraphs $H$ were considered by Huang, Lee and Sudakov [24], who proved an analogue of the bandwidth theorem (Theorem 1) in $G(n, p)$ with $0<p<1$ constant (for subgraphs $H$ with certain vertices not in triangles). A version which works for much smaller probabilities $p \gg\left(\frac{\log n}{n}\right)^{1 / \Delta}$ in the special case of bipartite graphs $H$ on $(1-o(1)) n$ vertices (with maximum degree $\Delta$ and sublinear bandwidth) was established in [10]. In [2, Theorem 1.9] the so-called sparse blow-up lemma is used to prove a similar result for graphs $H$ which are not necessarily bipartite. An even better bound on $p$ was obtained when we restrict the problem to the class of almost spanning trees $H$ : Balogh, Csaba and Samotij [6] proved that the local resilience of $G(n, p)$ with respect to containing copies of all trees $T$ on $(1-o(1)) n$ vertices with $\Delta(T) \leq \Delta$ is asymptotically almost surely at least $1 / 2-o(1)$ if $p \gg 1 / n$, which is optimal. Finally, returning to $H$-factors, Conlon, Gowers, Samotij and Schacht [15] gave resilience results for almost-spanning $H$-factors which work down to the optimal probability threshold, but leave a linear number of vertices uncovered; Nenadov and Škorić [36] substantially improved this, but (for most graphs) the number of vertices left uncovered in their result is still not the correct order of magnitude.

\section{Our results.}

We prove several sparse analogues of the bandwidth theorem (Theorem 1). Our first result is a version for sparse random graphs, extending the resilience results of Huang, Lee and Sudakov [24], [10], and [2]. 
Theorem 7. For each $\gamma>0, \Delta \geq 2$, and $k \geq 1$, there exist constants $\beta^{*}>0$ and $C^{*}>0$ such that the following holds asymptotically almost surely for $\Gamma=G(n, p)$ if $p \geq C^{*}\left(\frac{\log n}{n}\right)^{1 / \Delta}$. Let $G$ be a spanning subgraph of $\Gamma$ with $\delta(G) \geq\left(\frac{k-1}{k}+\gamma\right) p n$, and let $H$ be a $k$-colourable graph on $n$ vertices with $\Delta(H) \leq \Delta$, bandwidth at most $\beta^{*} n$, and with at least $C^{*} p^{-2}$ vertices which are not contained in any triangles of $H$. Then $G$ contains a copy of $H$.

Observe that the bound on $p$ achieved in this result matches the bound in the universality result in Theorem 5. Hence, though we do not believe it to be optimal, improving it will most likely be hard. Moreover, as explained in conjunction with Theorem 1, the minimum degree of $G$ cannot be decreased, nor can the bandwidth restriction be removed. As indicated above, it is also necessary that $\Theta\left(p^{-2}\right)$ vertices of $H$ are not in triangles.

If in addition the subgraph $H$ is also $D$-degenerate, we can prove a variant of Theorem 7 for $p \gg(\log n / n)^{1 /(2 D+1)}$. Again, this probability bound matches the one in the currently best universality result for $D$-degenerate graphs given in Theorem 6 . As before we require a certain number of vertices which are not in triangles of $H$. But, due to technicalities of our proof method, in addition these vertices are now also required not to be in four-cycles.

Theorem 8. For each $\gamma>0, \Delta \geq 2$, and $D, k \geq 1$, there exist constants $\beta^{*}>0$ and $C^{*}>0$ such that the following holds asymptotically almost surely for $\Gamma=G(n, p)$ if $p \geq C^{*}\left(\frac{\log n}{n}\right)^{1 /(2 D+1)}$. Let $G$ be a spanning subgraph of $\Gamma$ with $\delta(G) \geq\left(\frac{k-1}{k}+\gamma\right)$ pn and let $H$ be a D-degenerate, $k$-colourable graph on $n$ vertices with $\Delta(H) \leq \Delta$, bandwidth at most $\beta^{*} n$ and with at least $C^{*} p^{-2}$ vertices which are not contained in any triangles or four-cycles of $H$. Then $G$ contains a copy of $H$.

Since trees are 1-degenerate this implies a resilience result for trees when $p \gg\left(\frac{\log n}{n}\right)^{1 / 3}$. This probability bound is much worse than that obtained by Balogh, Csaba, and Samotij [6] for almostspanning trees, and unlikely to be optimal, but it is the first resilience result for bounded degree spanning trees in $G(n, p)$.

Finally, we also establish a sparse analogue of Theorem 1 in bijumbled graphs, one of the most widely studied classes of pseudorandom graphs. A graph $\Gamma$ is $(p, v)$-bijumbled if for all disjoint sets $X, Y \subseteq V(\Gamma)$ we have

$$
|e(X, Y)-p| X|| Y|| \leq v \sqrt{|X||Y|} .
$$

This definition goes back to an equivalent notion introduced by Thomason [42] who initiated the study of pseudorandom graphs. It is also related to the well investigated class of $(n, d, \lambda)$-graphs in that an $(n, d, \lambda)$-graph is $\left(\frac{d}{n}, \lambda\right)$-bijumbled.

Only very recently a universality result similar to Theorem 5 was established for bijumbled graphs in [2], where it was shown that $(p, v)$-bijumbled graphs $G$ with $\delta(G) \geq \frac{1}{2} p n$ and $v \ll p^{\max (4,(3 \Delta+1) / 2)} n$ are universal for $\mathcal{H}(n, \Delta)$. Our resilience result works for the same bijumbledness condition, though we do not believe it to be optimal. Local resilience results in bijumbled graphs were so far only obtained for special subgraphs $H$ : Dellamonica, Kohayakawa, Marciniszyn, and Steger [19] considered cycles $H$ of length $(1-o(1)) n$, the results of Conlon, Fox and Zhao [18] imply resilience for $F$-factors covering $(1-o(1)) n$ vertices, and Krivelevich, Lee and Sudakov [31] established a resilience result for pancyclicity. Hence, previous to this work only little was known about the resilience of bijumbled (or indeed any other common notion of pseudorandom) graphs. 
Theorem 9. For each $\gamma>0, \Delta \geq 2$, and $k \geq 1$, there exists a constant $c>0$ such that the following holds for any $p>0$. Given $v \leq c p^{\max (4,(3 \Delta+1) / 2)} n$, suppose $\Gamma$ is a $(p, v)$-bijumbled graph, $G$ is a spanning subgraph of $\Gamma$ with $\delta(G) \geq\left(\frac{k-1}{k}+\gamma\right) p n$, and $H$ is a k-colourable graph on $n$ vertices with $\Delta(H) \leq \Delta$ and bandwidth at most cn. Suppose further that there are at least $c^{-1} p^{-6} v^{2} n^{-1}$ vertices in $V(H)$ that are not contained in any triangles of $H$. Then $G$ contains a copy of $H$.

We remark that the requirement of $C^{*} p^{-6} v^{2} n^{-1}$ vertices of $H$ not being in triangles comes from our use of a so-called regularity inheritance lemma proved in [3]; this bound is not believed to be optimal (see Section 11 for further details).

The proofs of our results rely on sparse versions of the so-called blow-up lemma. The blow-up lemma is an important tool in extremal graph theory, proved by Komlós, Sárközy and Szemerédi [30] and was for example instrumental in the proof of the bandwidth theorem and its analogue in $G(n, p)$ for constant $p$ by Huang, Lee and Sudakov [24]. However it applies only to dense graphs. Several of the earlier resilience results in sparse random graphs developed sparse blow-up type results handling special classes of graphs: Balogh, Lee and Samotij [7] proved a sparse blow-up lemma for embedding triangle factors, and in [10] a blow-up lemma for embedding almost spanning bipartite graphs in sparse graphs was used. Full versions of the blow-up lemma in sparse random graphs and pseudorandom graphs were established only very recently in [2]. We will use these here. We remark that a simple use of these blow-up lemmas gives almost spanning versions of our main results (as already noted in [2]), and the main work here is to extend this to spanning embedding results, which turns out to be much harder.

Further, we note that we actually prove somewhat stronger statements than Theorem 7 , Theorem 8 , and Theorem 9 in the same sense in that a stronger statement than Theorem 1 was proven in [12]: we allow $H$ in fact to be $(k+1)$-colourable, where the additional colour may only be assigned to very few well distributed vertices (for details see, e.g., Theorem 25 below). Thus, for instance, even though Theorem 7 only implies that the local resilience of $G(n, p)$ with respect to Hamiltonicity is a.a.s. $\frac{1}{2}-o(1)$ when $n$ is even, Theorem 25 implies it also for $n$ odd.

\section{Organisation.}

The remainder of this paper is organised as follows. In Section 2 we introduce necessary definitions and collect some known results which we need in our proofs. Next, in Section 3, we outline the proof of the bandwidth theorem in sparse random graphs, Theorem 7 , and state the four technical lemmas we require. Their proofs are given in Sections 4-7, and the proof of Theorem 7 is presented in Section 8. We provide the modifications required to obtain Theorem 8 in Section 9, and those required for Theorem 9 in Section 10. Finally, Section 11 contains some concluding remarks, and Appendix A contains proofs of a few results which are more or less standard but which we could not find in the form we need in the literature.

\section{Preliminaries}

Throughout the paper log denotes the natural logarithm. We assume that the order $n$ of all graphs tends to infinity and therefore is sufficiently large whenever necessary. For reals $a, b>0$ and integer $k \in \mathbb{N}$, we use the notation $(a \pm b)=[a-b, a+b]$ and $[k]=\{1, \ldots, k\}$. Our graph-theoretic notation is 
THE BANDWIDTH THEOREM IN SPARSE GRAPHS

standard and follows [8]. In particular, given a graph $G$ its vertex set is denoted by $V(G)$ and its edge set by $E(G)$. Let $A, B \subseteq V$ be disjoint vertex sets. We denote the number of edges between $A$ and $B$ by $e(A, B)$. For a vertex $v \in V(G)$ we write $N_{G}(v)$ for the neighbourhood of $v$ in $G$ and $N_{G}(v, A):=$ $N_{G}(v) \cap A$ for the neighbourhood of $v$ restricted to $A$ in $G$. Given vertices $v_{1}, \ldots, v_{k} \in V(G)$ we denote the joint neighbourhood of $v_{1}, \ldots, v_{k}$ restricted to a set $A$ by $N_{G}\left(v_{1}, \ldots, v_{k} ; A\right)=\bigcap_{i \in[k]} N_{G}\left(v_{i}, A\right)$. Finally, we use the notation $\operatorname{deg}_{G}(v):=\left|N_{G}(v)\right|$ and $\operatorname{deg}_{G}(v, A):=\left|N_{G}(v, A)\right|$, as well as $\operatorname{deg}_{G}\left(v_{1}, \ldots, v_{k} ; A\right):=$ $\left|N_{G}\left(v_{1}, \ldots, v_{k} ; A\right)\right|$ for the degree of $v$ in $G$, the degree of $v$ restricted to $A$ in $G$ and the size of the joint neighbourhood of $v_{1}, \ldots, v_{k}$ restricted to $A$ in $G$. Finally, let $\operatorname{deg}_{G}(v):=\left|N_{G}(v)\right|$ be the degree of $v$ in $G$. For the sake of readability, we do not intend to optimise the constants in our theorems and proofs.

Now we introduce some definitions and results of the regularity method as well as related tools that are essential in our proofs. In particular, we state a minimum degree version of the sparse regularity lemma (Lemma 13) and the sparse blow up lemma (Lemma 16). Both lemmas use the concept of regular pairs. Let $G=(V, E)$ be a graph, $\varepsilon, d>0$, and $p \in(0,1]$. Moreover, let $X, Y \subseteq V$ be two disjoint nonempty sets. The $p$-density of the pair $(X, Y)$ is defined as

$$
d_{G, p}(X, Y):=\frac{e_{G}(X, Y)}{p|X||Y|} .
$$

For most of this paper, when we work with random graphs, we will be interested in the regularity concept called lower-regularity. When we work with bijumbled graphs, on the other hand, we will need the stronger concept regularity. The difference is that in the former we impose only lower bounds on $p$-densities, whereas in the latter we impose in addition upper bounds. The main reason for this difference is that our 'regularity inheritance lemmas' below have different requirements in random and in bijumbled graphs; we do not otherwise make use of the extra strength of 'regular' as opposed to 'lower-regular'.

We also need to define super-regularity, for which we require $G$ to be a subgraph of a graph $\Gamma$, which will be the random or bijumbled graph whose resilience properties we are establishing.

Definition $10((\varepsilon, d, p)$-(super-)(lower-)regular pairs). Let $G$ and $\Gamma$ be graphs with $G \subseteq \Gamma$. The pair

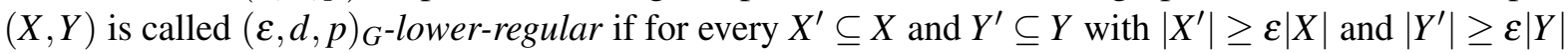
we have $d_{G, p}\left(X^{\prime}, Y^{\prime}\right) \geq d-\varepsilon$.

It is called $(\varepsilon, d, p)_{G}$-regular if there exists $d^{\prime} \geq d$ such that for every $X^{\prime} \subseteq X$ and $Y^{\prime} \subseteq Y$ with $\left|X^{\prime}\right| \geq \varepsilon|X|$ and $\left|Y^{\prime}\right| \geq \varepsilon|Y|$ we have $d_{G, p}\left(X^{\prime}, Y^{\prime}\right)=d^{\prime} \pm \varepsilon$.

If $(X, Y)$ is either $(\varepsilon, d, p)_{G}$-lower-regular or $(\varepsilon, d, p)_{G}$-regular, and in addition we have

$$
\begin{aligned}
& \left|N_{G}(x, Y)\right| \geq(d-\varepsilon) \max \left(p|Y|, \operatorname{deg}_{\Gamma}(x, Y) / 2\right) \quad \text { and } \\
& \left|N_{G}(y, X)\right| \geq(d-\varepsilon) \max \left(p|X|, \operatorname{deg}_{\Gamma}(y, X) / 2\right)
\end{aligned}
$$

for every $x \in X$ and $y \in Y$, then the pair $(X, Y)$ is called $(\varepsilon, d, p)_{G}$-super-regular. When we use superregularity it will be clear from the context whether $(X, Y)$ is lower-regular or regular.

Note that a regular pair is by definition lower-regular, though the converse does not hold. Furthermore, although the definition of super-regularity of $G$ contains a reference to $\Gamma$, at each place in this paper where we use super-regularity, we will see that the first term in the maximum is larger than the second. When it is clear from the context, we may omit the subscript $G$ in $(\varepsilon, d, p)_{G^{-}}$(super-)regular which is 
used to indicate with respect to which graph a pair is (super-)regular. A direct consequence of the definition of $(\varepsilon, d, p)$-lower-regular pairs is the following proposition about the sizes of neighbourhoods in lower-regular pairs.

Proposition 11. Let $(X, Y)$ be $(\varepsilon, d, p)$-lower-regular. Then there are less than $\varepsilon|X|$ vertices $x \in X$ with $|N(x, Y)|<(d-\varepsilon) p|Y|$.

The next proposition asserts that altering the vertex sets in an $(\varepsilon, d, p)$-(lower-)regular pair slightly does not destroy (lower-)regularity.

Proposition 12. Let $(X, Y)$ be an $(\varepsilon, d, p)$-lower-regular pair in a graph $G$ and let $\hat{X}$ and $\hat{Y}$ be two subsets of $V(G)$ such that $|X \triangle \hat{X}| \leq \mu|X|$ and $|Y \triangle \hat{Y}| \leq v|Y|$ for some $0 \leq \mu, v \leq 1$. Then $(\hat{X}, \hat{Y})$ is $(\hat{\varepsilon}, d, p)$-lower-regular, where $\hat{\varepsilon}:=\varepsilon+2 \sqrt{\mu}+2 \sqrt{v}$. Furthermore, if for any disjoint $A, A^{\prime} \subseteq V(G)$ with $|A| \geq \mu|X|$ and $\left|A^{\prime}\right| \geq v|Y|$ we have $e\left(A, A^{\prime}\right) \leq(1+\mu+v) p|A|\left|A^{\prime}\right|$, and $(X, Y)$ is $(\varepsilon, d, p)$-regular, then $(\hat{X}, \hat{Y})$ is $(\hat{\varepsilon}, d, p)$-regular.

We defer the proof of this to Appendix A.

In order to state the sparse regularity lemma, we need some more definitions. A partition $\mathcal{V}=$ $\left\{V_{i}\right\}_{i \in\{0, \ldots, r\}}$ of the vertex set of $G$ is called an $(\varepsilon, p)_{G^{-}}$regular partition of $V(G)$ if $\left|V_{0}\right| \leq \varepsilon|V(G)|$

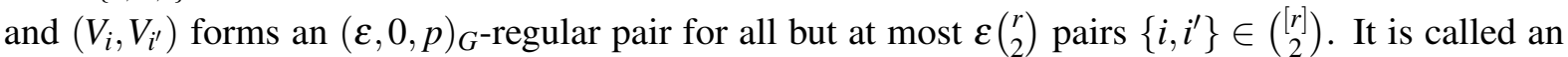
equipartition if $\left|V_{i}\right|=\left|V_{i^{\prime}}\right|$ for every $i, i^{\prime} \in[r]$. The partition $\mathcal{V}$ is called $(\varepsilon, d, p)$-(lower-)regular on a graph $R$ with vertex set $[r]$ if $\left(V_{i}, V_{i^{\prime}}\right)$ is $(\varepsilon, d, p)_{G^{-}}$(lower-)regular for every $\left\{i, i^{\prime}\right\} \in E(R)$. The graph $R$ is referred to as the $(\varepsilon, d, p)_{G}$-reduced graph of $\mathcal{V}$, the partition classes $V_{i}$ with $i \in[r]$ as clusters, and $V_{0}$ as the exceptional set. We also say that $\mathcal{V}$ is $(\varepsilon, d, p)_{G}$-super-regular on a graph $R^{\prime}$ with vertex set $[r]$ if $\left(V_{i}, V_{i^{\prime}}\right)$ is $(\varepsilon, d, p)_{G^{-}}$-super-regular for every $\left\{i, i^{\prime}\right\} \in E\left(R^{\prime}\right)$. Again, when we talk about reduced graphs or super-regularity, whether we are using lower-regularity or regularity will be clear from the context. We will however always specify whether a partition is regular or only lower-regular on $R$.

Analogously to Szemerédi's regularity lemma for dense graphs, the sparse regularity lemma, proved by Kohayakawa and Rödl [28, 29], asserts the existence of an $(\varepsilon, p)$-regular partition of constant size of any sparse graph. We state a minimum degree version of this lemma, whose proof (following [10]) we defer to Appendix A.

Lemma 13 (Minimum degree version of the sparse regularity lemma). For each $\varepsilon>0$, each $\alpha \in[0,1]$, and $r_{0} \geq 1$ there exists $r_{1} \geq 1$ with the following property. For any $d \in[0,1]$, any $p>0$, and any $n$-vertex graph $G$ with minimum degree $\alpha$ pn such that for any disjoint $X, Y \subseteq V(G)$ with $|X|,|Y| \geq \frac{\varepsilon n}{r_{1}}$ we have $e(X, Y) \leq\left(1+\frac{1}{1000} \varepsilon^{2}\right) p|X||Y|$, there is an $(\varepsilon, p)_{G}$-regular equipartition of $V(G)$ with $(\varepsilon, d, p)_{G}$-reduced graph $R$ satisfying $\delta(R) \geq(\alpha-d-\varepsilon)|V(R)|$ and $r_{0} \leq|V(R)| \leq r_{1}$.

A key ingredient in the proof of our main theorem is the so-called sparse blow up lemma developed by Hàn, Kohayakawa, Person, and two of the current authors in [2]. Given a subgraph $G \subseteq \Gamma=G(n, p)$ with $p \gg(\log n / n)^{1 / \Delta}$ and an $n$-vertex graph $H$ with maximum degree at most $\Delta$ with vertex partitions $\mathcal{V}$ and $\mathcal{W}$, respectively, the sparse blow up lemma guarantees under certain conditions a spanning embedding of $H$ in $G$ which respects the given partitions. In order to state this lemma we need to introduce some definitions. 
The BANDWIDTh TheOREM IN SPARSE GRAPHS

Definition $14\left(\left(\vartheta, R^{\prime}\right)\right.$-buffer). Let $R^{\prime}$ be a graph on $r$ vertices and let $H$ be a graph with vertex partition $\mathcal{W}=\left\{W_{i}\right\}_{i \in[r]}$. We say that the family $\widetilde{\mathcal{W}}=\left\{\widetilde{W}_{i}\right\}_{i \in[r]}$ of subsets $\widetilde{W}_{i} \subseteq W_{i}$ is an $\left(\vartheta, R^{\prime}\right)$-buffer for $H$ if

(i) $\left|\widetilde{W}_{i}\right| \geq \vartheta\left|W_{i}\right|$ for all $i \in[r]$, and

(ii) for each $i \in[r]$ and each $x \in \widetilde{W}_{i}$, the first and second neighbourhood of $x$ go along $R^{\prime}$, i.e., for each $\{x, y\},\{y, z\} \in E(H)$ with $y \in W_{j}$ and $z \in W_{k}$ we have $\{i, j\} \in E\left(R^{\prime}\right)$ and $\{j, k\} \in E\left(R^{\prime}\right)$.

Let $G$ and $H$ be graphs on $n$ vertices with partitions $\mathcal{V}=\left\{V_{i}\right\}_{i \in[r]}$ of $V(G)$ and $\mathcal{W}=\left\{W_{i}\right\}_{i \in[r]}$ of $V(H)$. We say that $\mathcal{V}$ and $\mathcal{W}$ are size-compatible if $\left|V_{i}\right|=\left|W_{i}\right|$ for all $i \in[r]$. If there exists an integer $m \geq 1$ such that $m \leq\left|V_{i}\right| \leq \kappa m$ for every $i \in[r]$, then we say that $(G, \mathcal{V})$ is $\kappa$-balanced. Given a graph $R$ on $r$ vertices, we call $(G, \mathcal{V})$ an $R$-partition if for every edge $\{x, y\} \in E(G)$ with $x \in V_{i}$ and $y \in V_{i^{\prime}}$ we have $\left\{i, i^{\prime}\right\} \in E(R)$.

We will actually need a little more than just an embedding of $H$ into $G$ respecting given partitions: we will need to restrict the images of some vertices of $H$ to subsets of the clusters of $G$. The following definition encapsulates the properties we have to guarantee for the sparse blow-up lemma to obtain such an embedding.

Definition 15 (Restriction pair). Let $\varepsilon, d>0, p \in[0,1]$, and let $R$ be a graph on $r$ vertices. Furthermore, let $G$ be a (not necessarily spanning) subgraph of $\Gamma=G(n, p)$ and let $H$ be a graph given with vertex partitions $\mathcal{V}=\left\{V_{i}\right\}_{i \in[r]}$ and $\mathcal{W}=\left\{W_{i}\right\}_{i \in[r]}$, respectively, such that $(G, \mathcal{V})$ and $(H, \mathcal{W})$ are size-compatible $R$-partitions. Let $\mathcal{J}=\left\{I_{x}\right\}_{x \in V(H)}$ be a collection of subsets of $V(G)$, called image restrictions, and $\mathcal{J}=\left\{J_{x}\right\}_{x \in V(H)}$ be a collection of subsets of $V(\Gamma) \backslash V(G)$, called restricting vertices. For each $i \in[r]$ we define $R_{i} \subseteq W_{i}$ to be the set of all vertices $x \in W_{i}$ for which $I_{x} \neq V_{i}$. We say that $\mathcal{J}$ and $\mathcal{J}$ are a $\left(\rho, \zeta, \Delta, \Delta_{J}\right)$-restriction pair if the following properties hold for each $i \in[r]$ and $x \in W_{i}$.

(RP 1) We have $\left|R_{i}\right| \leq \rho\left|W_{i}\right|$.

(RP2) If $x \in R_{i}$, then $I_{x} \subseteq \bigcap_{u \in J_{x}} N_{\Gamma}\left(u, V_{i}\right)$ is of size at least $\zeta(d p)^{\left|J_{x}\right|}\left|V_{i}\right|$.

(RP3) If $x \in R_{i}$, then $\left|J_{x}\right|+\operatorname{deg}_{H}(x) \leq \Delta$ and if $x \in W_{i} \backslash R_{i}$, then $J_{x}=\varnothing$.

(RP 4) Each vertex in $V(G)$ appears in at most $\Delta_{J}$ of the sets of $\mathcal{J}$.

(RP 5) We have $\left|\bigcap_{u \in J_{x}} N_{\Gamma}\left(u, V_{i}\right)\right|=(p \pm \varepsilon p)^{\left|J_{x}\right|}\left|V_{i}\right|$.

(RP6) If $x \in R_{i}$, for each $x y \in E(H)$ with $y \in W_{j}$,

$$
\text { the pair } \quad\left(V_{i} \cap \bigcap_{u \in J_{x}} N_{\Gamma}(u), V_{j} \cap \bigcap_{v \in J_{y}} N_{\Gamma}(v)\right) \quad \text { is }(\varepsilon, d, p)_{G} \text {-lower-regular. }
$$

Suppose $\mathcal{V}$ is an $(\varepsilon, d, p)_{G}$-lower-regular partition of $V(G)$ with reduced graph $R$, and let $R^{\prime}$ be a subgraph of $R$. We say $(G, \mathcal{V})$ has one-sided inheritance on $R^{\prime}$ if for every $\{i, j\},\{j, k\} \in E\left(R^{\prime}\right)$ and every $v \in V_{i}$ the pair $\left(N_{\Gamma}\left(v, V_{j}\right), V_{k}\right)$ is $(\varepsilon, d, p)_{G^{-}}$-lower-regular. Given a $\left(\vartheta, R^{\prime}\right)$-buffer $\widetilde{\mathcal{W}}$, we say that $(G, \mathcal{V})$ has two-sided inheritance on $R^{\prime}$ for $\widetilde{\mathcal{W}}$ if whenever there is a triangle $w_{i} w_{j} w_{k} \in H$ with $w_{i} \in \widetilde{W}_{i}, w_{j} \in W_{j}$ and $w_{k} \in W_{k}$, it follows that for every $v \in V_{i}$ the pair $\left(N_{\Gamma}\left(v, V_{j}\right), N_{\Gamma}\left(v, V_{k}\right)\right)$ is $(\varepsilon, d, p)_{G}$-lower-regular.

Now we can finally state the sparse blow up lemma. 
Lemma 16 ([2, Lemma 1.21]). For each $\Delta, \Delta_{R^{\prime}}, \Delta_{J}, \vartheta, \zeta, d>0, \kappa>1$ there exist $\varepsilon_{\mathrm{BL}}, \rho>0$ such that for all $r_{1}$ there is a $C_{\mathrm{BL}}$ such that for $p \geq C_{\mathrm{BL}}(\log n / n)^{1 / \Delta}$ the random graph $\Gamma=G_{n, p}$ asymptotically almost surely satisfies the following.

Let $R$ be a graph on $r \leq r_{1}$ vertices and let $R^{\prime} \subseteq R$ be a spanning subgraph with $\Delta\left(R^{\prime}\right) \leq \Delta_{R^{\prime}}$. Let $H$ and $G \subseteq \Gamma$ be graphs given with $\kappa$-balanced, size-compatible vertex partitions $\mathcal{W}=\left\{W_{i}\right\}_{i \in[r]}$ and $\mathcal{V}=\left\{V_{i}\right\}_{i \in[r]}$ with parts of size at least $m \geq n /\left(\kappa r_{1}\right)$. Let $\mathcal{J}=\left\{I_{x}\right\}_{x \in V(H)}$ be a family of image restrictions, and $\mathcal{J}=\left\{J_{x}\right\}_{x \in V(H)}$ be a family of restricting vertices. Suppose that

(BUL 1) $\Delta(H) \leq \Delta$, for every edge $\{x, y\} \in E(H)$ with $x \in W_{i}$ and $y \in W_{j}$ we have $\{i, j\} \in E(R)$ and $\widetilde{\mathcal{W}}=\left\{\widetilde{W}_{i}\right\}_{i \in[r]}$ is an $\left(\vartheta, R^{\prime}\right)$-buffer for $H$,

$(B U L 2) \mathcal{V}$ is $\left(\varepsilon_{\mathrm{BL}}, d, p\right)_{G}$-lower-regular on $R,\left(\varepsilon_{\mathrm{BL}}, d, p\right)_{G}$-super-regular on $R^{\prime}$, has one-sided inheritance on $R^{\prime}$, and two-sided inheritance on $R^{\prime}$ for $\widetilde{\mathcal{W}}$,

(BUL 3) J and J form a $\left(\rho, \zeta, \Delta, \Delta_{J}\right)$-restriction pair.

Then there is an embedding $\phi: V(H) \rightarrow V(G)$ such that $\phi(x) \in I_{x}$ for each $x \in H$.

Observe that in the blow up lemma for dense graphs, proved by Komlós, Sárközy, and Szemerédi [30], one does not need to explicitly ask for one- and two-sided inheritance properties since they are always fulfilled by dense regular partitions. This is, however, not true in general in the sparse setting. The following two lemmas will be very useful whenever we need to choose vertices whose neighbourhoods inherit lower-regularity.

Lemma 17 (One-sided lower-regularity inheritance, [2]). For each $\varepsilon_{\text {OSRIL }}, \alpha_{\text {OSRIL }}>0$ there exist $\varepsilon_{0}>0$ and $C>0$ such that for any $0<\varepsilon<\varepsilon_{0}$ and $0<p<1$ asymptotically almost surely $\Gamma=G(n, p)$ has the following property. For any disjoint sets $X$ and $Y$ in $V(\Gamma)$ with $|X| \geq C \max \left(p^{-2}, p^{-1} \log n\right)$ and

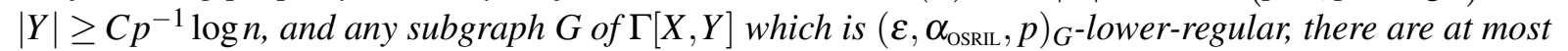
$C p^{-1} \log ($ en $/|X|)$ vertices $z \in V(\Gamma)$ such that $\left(X \cap N_{\Gamma}(z), Y\right)$ is not $\left(\varepsilon_{\text {OSRIL }}, \alpha_{\text {OSRIL }}, p\right)_{G}$-lower-regular.

Lemma 18 (Two-sided lower-regularity inheritance, [2]). For each $\varepsilon_{\mathrm{TSRI}}, \alpha_{\mathrm{TSRL}}>0$ there exist $\varepsilon_{0}>0$ and $C>0$ such that for any $0<\varepsilon<\varepsilon_{0}$ and $0<p<1$, asymptotically almost surely $\Gamma=G_{n, p}$ has the following property. For any disjoint sets $X$ and $Y$ in $V(\Gamma)$ with $|X|,|Y| \geq C \max \left\{p^{-2}, p^{-1} \log n\right\}$, and any subgraph $G$ of $\Gamma[X, Y]$ which is $\left(\varepsilon, \alpha_{\mathrm{TSRIL}}, p\right)_{G^{-}}$lower-regular, there are at most $C \max \left\{p^{-2}, p^{-1} \log (\right.$ en $\left./|X|)\right\}$ vertices $z \in V(\Gamma)$ such that $\left(X \cap N_{\Gamma}(z), Y \cap N_{\Gamma}(z)\right)$ is not $\left(\varepsilon_{\text {TSRIL }}, \alpha_{\text {TSRIL }}, p\right)_{G}$-lower-regular.

We close this section with some probabilistic tools. We start with the following useful observation. Roughly speaking, it states that a.a.s. nearly all vertices in $G(n, p)$ have approximately the expected number of neighbours within large enough subsets.

Proposition 19. For each $\varepsilon>0$ there exists a constant $C>0$ such that for every $0<p<1$ asymptotically almost surely $\Gamma=G(n, p)$ has the following properties. For any disjoint $X, Y \subseteq V(\Gamma)$ with $|X| \geq C p^{-1} \log n$ and $|Y| \geq C p^{-1} \log ($ en $/|X|)$, we have $e(X, Y)=(1 \pm \varepsilon) p|X||Y|$ and $e(X) \leq 2 p|X|^{2}$. Furthermore, for every $X \subseteq V(\Gamma)$ with $|X| \geq C p^{-1} \log n$, the number of vertices $v \in V(\Gamma)$ with ||$N_{\Gamma}(v, X) \mid-$ $p|X||>\varepsilon p| X \mid$ is at most $C p^{-1} \log (e n /|X|)$. 
Note that in most of this paper we will use the upper bound $\log (e n /|X|) \leq \log n$ when applying this proposition, and Lemmas 17 and 18, valid since (in all applications) we have $|X| \geq e$. We will only need the full strength of these three results when proving the Lemma for $G$ (Lemma 26).

In the proof of Proposition 19 we use the following version of Chernoff's Inequalities (see e.g. [25, Chapter 2] for a proof).

Theorem 20 (Chernoff's Inequality, [25]). Let $X$ be a random variable which is the sum of independent Bernoulli random variables. Then we have for $\varepsilon \leq 3 / 2$

$$
\mathbb{P}[|X-\mathbb{E}[X]|>\varepsilon \mathbb{E}[X]]<2 e^{-\varepsilon^{2} \mathbb{E}[X] / 3} .
$$

Furthermore, if $t \geq 6 \mathbb{E}[X]$ then we have

$$
\mathbb{P}[X \geq \mathbb{E}[X]+t] \leq e^{-t} .
$$

Proof of Proposition 19. Since the statement of the proposition is stronger when $\varepsilon$ is smaller, we may assume that $0<\varepsilon \leq 1$. We set $C^{\prime}=100 \varepsilon^{-2}$ and $C=1000 C^{\prime} \varepsilon^{-1}$.

We first show that $\Gamma=G(n, p)$ a.a.s. has the following two properties. For any disjoint $A, B \subseteq V(\Gamma)$, with $|A| \geq C^{\prime} p^{-1} \log n$ and $|B| \geq C^{\prime} p^{-1} \log (e n /|A|)$, we have $e(A, B)=\left(1 \pm \frac{\varepsilon}{2}\right) p|A||B|$. For any $A \subseteq V(\Gamma)$, we have $e(A) \leq 4 p|A|^{2}+2|A| \log n$, and if $|A| \geq C^{\prime} p^{-1} \log n$ then $e(A) \leq 2 p|A|^{2}$. Note that these properties imply the first two conclusions of the proposition.

We estimate the failure probability of the first property using Theorem 20 and the union bound. Assuming without loss of generality that $|A| \geq|B|$, this probability is at most

$$
\begin{aligned}
\sum_{|A|,|B| \leq n}\left(\begin{array}{c}
n \\
|A|
\end{array}\right)^{2} \cdot 2 e^{-\varepsilon^{2} p|A||B| / 12} & \leq 2 n \sum_{|A|}\left(\frac{e n}{|A|}\right)^{2|A|} e^{-\varepsilon^{2} C^{\prime}|A| \log (e n /|A|) / 12} \\
& <2 n \sum_{|A|}\left(\frac{e n}{|A|}\right)^{-2|A|}
\end{aligned}
$$

For the second property, observe that $4 p|A|^{2}>7 p\left(\begin{array}{c}|A| \\ 2\end{array}\right)$, so that for any given $A$ by Theorem 20 we have

$$
\mathbb{P}\left[e(A) \geq 4 p|A|^{2}+2|A| \log n\right] \leq e^{-2|A| \log n}=n^{-2|A|} .
$$

Taking a union bound over the at most $n^{|A|}$ choices of $A$ given $|A|$, we see that the failure probability of the second property is at most $\sum_{a=1}^{n} n^{-a}$.

Finally, the failure probability of the last property is at most

$$
\sum_{|A| \geq C^{\prime} p^{-1} \log n} n^{|A|} \cdot 2 e^{-p\left(\begin{array}{c}
|A| \\
2
\end{array}\right) / 3} \leq \sum_{|A|} 2 n^{|A|} e^{-C^{\prime}|A| \log n / 12} \leq 2 n^{-2},
$$

and since all three failure probabilities tend to zero as $n \rightarrow \infty$, we conclude that a.a.s. $G(n, p)$ enjoys both properties.

Now suppose $\Gamma$ has these properties, and let $X \subseteq V(\Gamma)$ have size at least $C p^{-1} \log n$. We first show that there are at most $C^{\prime} p^{-1} \log (e n /|X|)$ vertices in $\Gamma$ which have less than $(1-\varepsilon) p|X|$ neighbours in $X$. 
If this were false, then we could choose a set $Y$ of $C^{\prime} p^{-1} \log (e n /|X|)$ vertices in $\Gamma$ which have less than $(1-\varepsilon) p|X|$ neighbours in $X$. By choice of $C$ and since $|X|>e$, we have $(1-\varepsilon) p|X| \leq\left(1-\frac{\varepsilon}{2}\right) p|X \backslash Y|$, so we see that $e(Y, X \backslash Y)<\left(1-\frac{\varepsilon}{2}\right) p|Y||X \backslash Y|$. This is a contradiction since $|X \backslash Y| \geq C^{\prime} p^{-1} \log n$.

Next we show that there are at most $2 C^{\prime} p^{-1} \log (e n /|X|)$ vertices of $\Gamma$ which have more than $(1+$ $\varepsilon) p|X|$ neighbours in $X$. Again, if this is not the case we can let $Y$ be a set of $2 C^{\prime} p^{-1} \log (e n /|X|)$ vertices of $\Gamma$ with more than $(1+\varepsilon) p|X|$ neighbours in $X$. Now $e(Y) \leq 4 p|Y|^{2}+2|Y| \log n=8 C^{\prime}|Y| \log (e n /|X|)+$ $2|Y| \log n \leq 10 C^{\prime}|Y| \log n$, so there are at most $|Y| / 2$ vertices in $Y$ which have $40 C^{\prime} \log n$ or more neighbours in $Y$. Let $Y^{\prime} \subseteq Y$ consist of those vertices with at most $40 C^{\prime} \log n$ neighbours in $Y$. For each $v \in Y^{\prime}$ we have

$$
(1+\varepsilon) p|X| \leq \operatorname{deg}(v ; X) \leq \operatorname{deg}(v ; Y)+\operatorname{deg}(v ; X \backslash Y),
$$

and so, by choice of $C$, each vertex of $Y^{\prime}$ has at least $\left(1+\frac{\varepsilon}{2}\right) p|X \backslash Y|$ neighbours in $X \backslash Y$. Since $\left|Y^{\prime}\right| \geq C^{\prime} p^{-1} \log (2 e n /|X|)$ and $|X \backslash Y| \geq|X| / 2 \geq C^{\prime} p^{-1} \log n$, this is a contradiction. Finally, since by choice of $C$ we have $3 C^{\prime} p^{-1} \log n<C p^{-1} \log n$ we conclude that all but at most $C p^{-1} \log (e n /|X|)$ vertices of $\Gamma$ have $(1 \pm \varepsilon) p|X|$ neighbours in $X$, as desired.

Now let $N, m$, and $s$ be positive integers and let $S$ and $S^{\prime} \subseteq S$ be two sets with $|S|=N$ and $\left|S^{\prime}\right|=m$. The hypergeometric distribution is the distribution of the random variable $X$ that is defined by drawing $s$ elements of $S$ without replacement and counting how many of them belong to $S^{\prime}$. It can be shown that Theorem 20 still holds in the case of hypergeometric distributions (see e.g. [25], Chapter 2 for a proof) with $\mathbb{E}[X]:=m s / N$.

Theorem 21 (Hypergeometric inequality, [25]). Let $X$ be a random variable that follows the hypergeometric distribution with parameters $N, m$, and $s$. Then for any $\varepsilon>0$ and $t \geq \varepsilon m s / N$ we have

$$
\mathbb{P}[|X-m s / N|>t]<2 e^{-\varepsilon^{2} t / 3}
$$

We require the following technical lemma, which is a consequence of the hypergeometric inequality stated in Theorem 21.

Lemma 22. For each $\eta>0$ and $\Delta$ there exists $C$ such that the following holds. Let $W \subseteq[n]$, let $t \leq 100 n^{\Delta}$, and let $T_{1}, \ldots, T_{t}$ be subsets of $W$. For each $m \leq|X|$ there is a set $S \subseteq W$ of size $m$ such that

$$
\left|T_{i} \cap S\right|=\frac{m}{|W|}\left|T_{i}\right| \pm\left(\eta\left|T_{i}\right|+C \log n\right) \text { for every } i \in[t]
$$

Proof. Set $C=30 \eta^{-2} \Delta$. Observe that for each $i$, the size of $T_{i} \cap S$ is hypergeometrically distributed. By Theorem 21, for each $i$ we have

$$
\mathbb{P}\left[\left|T_{i} \cap S\right| \neq \frac{m}{|W|}\left|T_{i}\right| \pm\left(\eta\left|T_{i}\right|+C \log n\right)\right]<2 e^{-\eta^{2} C \log n / 3}<\frac{2}{n^{1+\Delta}}
$$

so taking the union bound over all $i \in[t]$ we conclude that the probability of failure is at most $2 t / n^{1+\Delta} \leq$ $200 / n \rightarrow 0$ as $n \rightarrow \infty$, as desired.

We shall also use McDiarmid's Inequality. 
The BAndwidTh TheOREM In SPARse GRAPHS

Lemma 23 (McDiarmid's Inequality [35]). Let $X_{1}, \ldots, X_{k}$ be independent random variables, where $X_{i}$ takes values in a finite set $A_{i}$ for each $i \in[k]$. Suppose that a function $g: A_{1} \times \ldots \times A_{k} \rightarrow \mathbb{R}$ satisfies for each $i \in[k]$

$$
\sup _{x_{1}, \ldots, x_{k}, \hat{x}_{i}}\left|g\left(x_{1}, x_{2}, \ldots, x_{k}\right)-g\left(x_{1}, x_{2}, \ldots, x_{i-1}, \hat{x}_{i}, x_{i+1}, \ldots, x_{k}\right)\right| \leq c_{i} .
$$

Then, for any $\varepsilon>0$, we have

$$
\mathbb{P}\left[\left|\mathbb{E}\left[g\left(X_{1}, \ldots, X_{k}\right)\right]-g\left(X_{1}, \ldots, X_{k}\right)\right| \geq \varepsilon\right] \leq 2 \exp \left\{-\frac{2 \varepsilon^{2}}{\sum_{i \in[k]} c_{i}^{2}}\right\} .
$$

\section{Proof overview and main lemmas}

Theorem 7 is a corollary of the following more general Theorem 25, which we prove in Section 8 . We require one preliminary definition.

Definition 24 (Zero-free colouring). Let $H$ be a $(k+1)$-colourable graph on $n$ vertices and let $\mathcal{L}$ be a labelling of its vertex set by integers $1, \ldots, n$ of bandwidth at most $\beta n$. A proper $(k+1)$-colouring $\sigma: V(H) \rightarrow\{0, \ldots, k\}$ of its vertex set is said to be $(z, \beta)$-zero-free with respect to $\mathcal{L}$ if any $z$ consecutive blocks contain at most one block with colour zero, where a block is defined as a set of the form $\{(t-1) 4 k \beta n+1, \ldots, t 4 k \beta n\}$ with some $t \in[1 /(4 k \beta)]$, and a block with colour zero is a block in which at least one vertex is coloured with zero.

Theorem 25. For each $\gamma>0, \Delta \geq 2$, and $k \geq 2$, there exist constants $\beta>0, z>0$, and $C>0$ such that the following holds asymptotically almost surely for $\Gamma=G(n, p)$ if $p \geq C\left(\frac{\log n}{n}\right)^{1 / \Delta}$. Let $G$ be a spanning subgraph of $\Gamma$ with $\delta(G) \geq\left(\frac{k-1}{k}+\gamma\right)$ pn and let $H$ be a graph on $n$ vertices with $\Delta(H) \leq \Delta$ that has a labelling $\mathcal{L}$ of its vertex set of bandwidth at most $\beta n, a(k+1)$-colouring that is $(z, \beta)$-zero-free with respect to $\mathcal{L}$ and where the first $\sqrt{\beta} n$ vertices in $\mathcal{L}$ are not given colour zero and the first $\beta n$ vertices in $\mathcal{L}$ include $C p^{-2}$ vertices that are not contained in any triangles of $H$. Then $G$ contains a copy of $H$.

\subsection{Proof overview}

We now give a brief sketch of the proof of Theorem 25. Ultimately, our goal is to apply the sparse blow-up lemma, Lemma 16, to find an embedding of $H$ into $G$. Thus, the proof boils down to obtaining the required conditions. But there is a catch: this is not as such possible, as for any lower-regular partition of $G$ there can be $O\left(p^{-2}\right)$ exceptional vertices which are 'badly behaved' with respect to the partition. These vertices will never satisfy the conditions of the sparse blow-up lemma, and we will have to deal with them beforehand. We will do this by 'pre-embedding' some vertices of $H$ to cover the exceptional vertices, and then apply the sparse blow-up lemma to complete the embedding of $H$ into $G$, using image restrictions to ensure we really obtain an embedding of $H$. Let us now fill in a few more details.

We start by obtaining, in the lemma for $G$, Lemma 26, a lower-regular partition of $G$ into parts $V_{0}$ and $V_{i, j}$ for $i \in[r]$ (where $r$ may be large but is bounded above by a constant) and $j \in[k]$ with several extra properties. The most important properties are that $\left|V_{0}\right|=O\left(p^{-2}\right)$, that the corresponding reduced graph, 
which we call $R_{r}^{k}$, on the vertex set $[r] \times[k]$ has high minimum degree and contains a so-called backbone graph on which the partition does not merely provide lower-regular pairs but super-regular pairs, and that all vertices outside $V_{0}$ have inheritance properties with respect to all lower-regular pairs. Here, a backbone graph has all edges between $(i, j)$ and $\left(i^{\prime}, j^{\prime}\right)$ with $\left|i-i^{\prime}\right| \leq 1$ and $j \neq j^{\prime}$. One should think of the backbone graph as consisting of copies of $K_{k}$ (one for each $i \in[r]$ ) connected in a linear order; and the high minimum degree of $R_{r}^{k}$ ensures that each $K_{k}$ extends to $K_{k+1}$ in $R_{r}^{k}$. In short, if the exceptional vertices $V_{0}$ did not exist, this partition, together with a corresponding partition of $V(H)$, would be what we need to apply the sparse blow-up lemma.

Passing over for now the inconvenient existence of $V_{0}$, our next task is to find the corresponding partition of $V(H)$, for which we use the lemma for $H$, Lemma 27. The basic idea is then to split $H$ into intervals in the bandwidth order. We assign the first interval to the first $K_{k}$ of the backbone graph according to the given colouring of $H$, with the few vertices of colour zero assigned to a vertex extending this clique of the backbone graph to $K_{k+1}$, and so on. Using the bandwidth property and zero-freeness of the colouring one can do this in such a way as to obtain a graph homomorphism from $H$ to $R_{r}^{k}$, which is what we need. In addition, we need the number of vertices assigned to each $(i, j) \in V\left(R_{r}^{k}\right)$ to be very close to $\left|V_{i, j}\right|$. We cannot guarantee exact equality, but we can get very close by making further use of bandwidth, zero-freeness, and the fact that $K_{k} \mathrm{~s}$ in $R_{r}^{k}$ extend to $K_{k+1} \mathrm{~s}$.

Now we have to deal with the exceptional set $V_{0}$. We do this as follows. We choose a vertex $v$ in the exceptional set, and 'pre-embed' to it a vertex $x$ picked from the first $\sqrt{\beta} n$ vertices of $\mathcal{L}$ which is not in any triangle of $H$. Using the common neighbourhood lemma, Lemma 28, we choose $\Delta$ neighbours of $v$ which are 'well-behaved' with respect to the clusters $V_{i, j}$ for some $i \in[r]$, and pre-embed the neighbours of $x$ to these vertices. The 'well-behaved' properties are what we need to generate image restrictions for the second neighbours of $x$ (which we will embed using the sparse blow-up lemma) satisfying the restriction pair properties. We also need to change the assignment from the Lemma for $H$ locally (up to a large but constant distance from $x$ ) to accommodate this: the vertex $x$, and its first and second neighbours, might have been assigned somewhere quite different previously. We repeat this until we have pre-embedded to all exceptional vertices, and let $H^{\prime}$ and $G^{\prime}$ be respectively the unembedded vertices of $H$ and the vertices of $G$ to which we did not pre-embed.

At this point we have all the conditions we need to apply the sparse blow-up lemma to complete the embedding, except that the partitions of $H^{\prime}$ and $G^{\prime}$ we have do not quite have parts of matching sizes. We use the balancing lemma, Lemma 29, to deal with this. The idea is simple: we take some carefully selected vertices in clusters of $G$ which are too big (compared to the assigned part of $H$ ) and move them to other clusters, first in order to make sure that the total number of vertices in $\bigcup_{i} V_{i, j}$ is correct for each $j$ (using the high minimum degree of $R_{r}^{k}$ ) and then (using the structure of the backbone graph) to give each cluster the correct size.

At last, applying the sparse blow-up lemma, Lemma 16, we complete the embedding of $H$ into $G$.

We note that this proof sketch glosses over some subtleties. In particular, at the two places where 'we choose' vertices onto which to pre-embed, we have to be quite careful to choose vertices correctly so that this strategy can be completed and we do not destroy good properties obtained earlier. We will return to this point immediately before the proof of Theorem 25 in Section 8 to explain how we do this. 
THE BANDWIDTH THEOREM IN SPARSE GRAPHS

\subsection{Main lemmas}

In this subsection we formulate the four main lemmas that we use in the proof of Theorem 25 mentioned in the above overview. We defer the proofs of these lemmas to later sections. Before stating these lemmas, we need some more definitions.

Let $r, k \geq 1$ and let $B_{r}^{k}$ be the backbone graph on $k r$ vertices. That is, we have

$$
V\left(B_{r}^{k}\right):=[r] \times[k]
$$

and for every $j \neq j^{\prime} \in[k]$ we have $\left\{(i, j),\left(i^{\prime}, j^{\prime}\right)\right\} \in E\left(B_{r}^{k}\right)$ if and only if $\left|i-i^{\prime}\right| \leq 1$.

Let $K_{r}^{k} \subseteq B_{r}^{k}$ be the spanning subgraph of $B_{r}^{k}$ that is the disjoint union of $r$ complete graphs on $k$ vertices given by the following components: the complete graph $K_{r}^{k}[\{(i, 1), \ldots,(i, k)\}]$ is called the $i$-th component of $K_{r}^{k}$ for each $i \in[r]$.

A vertex partition $\mathcal{V}^{\prime}=\left\{V_{i, j}\right\}_{i \in[r], j \in[k]}$ is called k-equitable if ||$V_{i, j}|-| V_{i, j^{\prime}}|| \leq 1$ for every $i \in[r]$ and $j, j^{\prime} \in[k]$. Similarly, an integer partition $\left\{n_{i, j}\right\}_{i \in[r], j \in[k]}$ of $n$ (meaning that $n_{i, j} \in \mathbb{Z}_{\geq 0}$ for every $i \in[r], j \in[k]$ and $\left.\sum_{i \in[r] j \in[k]} n_{i, j}=n\right)$ is $k$-equitable if $\left|n_{i, j}-n_{i, j^{\prime}}\right| \leq 1$ for every $i \in[r]$ and $j, j^{\prime} \in[k]$.

The lemma for $G$ says that a.a.s. $\Gamma=G(n, p)$ satisfies the following property if $p \gg(\log n / n)^{1 / 2}$. For any spanning subgraph $G \subseteq \Gamma$ with minimum degree a sufficiently large fraction of $p n$, there exists an $(\varepsilon, d, p)_{G}$-lower-regular vertex partition $\mathcal{V}$ of $V(G)$ whose reduced graph $R_{r}^{k}$ contains a clique factor $K_{r}^{k}$ on which the corresponding vertex sets of $\mathcal{V}$ are pairwise $(\varepsilon, d, p)$-super-regular. Furthermore, $(G, \mathcal{V})$ has one-sided and two-sided inheritance with respect to $R_{r}^{k}$, and the $\Gamma$-neighbourhoods of all vertices but the ones in the exceptional set of $\mathcal{V}$ have almost exactly their expected size in each cluster. The proof of Lemma 26 is given in Section 4.

Lemma 26 (Lemma for $G$ ). For each $\gamma>0$ and integers $k \geq 2$ and $r_{0} \geq 1$ there exists $d>0$ such that for every $\varepsilon \in\left(0, \frac{1}{2 k}\right)$ there exist $r_{1} \geq 1$ and $C^{*}>0$ such that the following holds a.a.s. for $\Gamma=G(n, p)$ if $p \geq C^{*}(\log n / n)^{1 / 2}$. Let $G=(V, E)$ be a spanning subgraph of $\Gamma$ with $\delta(G) \geq\left(\frac{k-1}{k}+\gamma\right) p n$. Then there exists an integer $r$ with $r_{0} \leq k r \leq r_{1}$, a subset $V_{0} \subseteq V$ with $\left|V_{0}\right| \leq C^{*} p^{-2}$, a $k$-equitable vertex partition $\mathcal{V}=\left\{V_{i, j}\right\}_{i \in[r], j \in[k]}$ of $V(G) \backslash V_{0}$, and a graph $R_{r}^{k}$ on the vertex set $[r] \times[k]$ with $K_{r}^{k} \subseteq B_{r}^{k} \subseteq R_{r}^{k}$, with $\delta\left(R_{r}^{k}\right) \geq\left(\frac{k-1}{k}+\frac{\gamma}{2}\right) k r$, and such that the following are true.

(G1) $\frac{n}{4 k r} \leq\left|V_{i, j}\right| \leq \frac{4 n}{k r}$ for every $i \in[r]$ and $j \in[k]$,

$(G 2) \mathcal{V}$ is $(\varepsilon, d, p)_{G}$-lower-regular on $R_{r}^{k}$ and $(\varepsilon, d, p)_{G}$-super-regular on $K_{r}^{k}$,

(G3) both $\left(N_{\Gamma}\left(v, V_{i, j}\right), V_{i^{\prime}, j^{\prime}}\right)$ and $\left(N_{\Gamma}\left(v, V_{i, j}\right), N_{\Gamma}\left(v, V_{i^{\prime}, j^{\prime}}\right)\right)$ are $(\varepsilon, d, p)_{G^{-}}$-lower-regular pairs for every $\left\{(i, j),\left(i^{\prime}, j^{\prime}\right)\right\} \in E\left(R_{r}^{k}\right)$ and $v \in V \backslash V_{0}$,

(G4) $\left|N_{\Gamma}\left(v, V_{i, j}\right)\right|=(1 \pm \varepsilon) p\left|V_{i, j}\right|$ for every $i \in[r], j \in[k]$ and every $v \in V \backslash V_{0}$.

Furthermore, if we replace $(G 3)$ with the weaker

$\left(G 3^{\prime}\right)\left(N_{\Gamma}\left(v, V_{i, j}\right), V_{i^{\prime}, j^{\prime}}\right)$ is an $(\varepsilon, d, p)_{G^{-}}$-lower-regular pair for every $\left\{(i, j),\left(i^{\prime}, j^{\prime}\right)\right\} \in E\left(R_{r}^{k}\right)$ and $v \in$ $V \backslash V_{0}$,

then we have the stronger bound $\left|V_{0}\right| \leq C^{*} p^{-1}$. 
After Lemma 26 has constructed a lower-regular partition $\mathcal{V}$ of $V(G)$, the second main lemma deals with the graph $H$ that we would like to find as a subgraph of $G$. More precisely, Lemma 27 provides a homomorphism $f$ from the graph $H$ to the reduced graph $R_{r}^{k}$ given by Lemma 26 which has among others the following properties. The edges of $H$ are mapped to the edges of $R_{r}^{k}$, and the vast majority of the edges of $H$ are assigned to edges of the clique factor $K_{r}^{k} \subseteq R_{r}^{k}$. The number of vertices of $H$ mapped to a vertex of $R_{r}^{k}$ only differs slightly from the size of the corresponding cluster of $\mathcal{V}$. The lemma further guarantees that each of the first $\sqrt{\beta} n$ vertices of the bandwidth ordering of $V(H)$ is mapped to $(1, j)$ with $j$ being the colour that the vertex has received by the given colouring of $H$. In case $H$ is $D$-degenerate the next lemma also ensures that for every $(i, j) \in[r] \times[k]$, a constant fraction of vertices mapped to $(i, j)$ have each at most $2 D$ neighbours.

Lemma 27 (Lemma for $H$ ). Given $D, k, r \geq 1$ and $\xi, \beta>0$ the following holds if $\xi \leq 1 /(k r)$ and $\beta \leq 10^{-10} \xi^{2} /\left(D k^{4} r\right)$. Let $H$ be a $D$-degenerate graph on $n$ vertices, let $\mathcal{L}$ be a labelling of its vertex set of bandwidth at most $\beta n$ and let $\sigma: V(H) \rightarrow\{0, \ldots k\}$ be a proper $(k+1)$-colouring that is $(10 / \xi, \beta)$ zero-free with respect to $\mathcal{L}$, where the colour zero does not appear in the first $\sqrt{\beta} n$ vertices of $\mathcal{L}$. Furthermore, let $R_{r}^{k}$ be a graph on vertex set $[r] \times[k]$ with $K_{r}^{k} \subseteq B_{r}^{k} \subseteq R_{r}^{k}$ such that for every $i \in[r]$ there exists a vertex $z_{i} \in([r] \backslash\{i\}) \times[k]$ with $\left\{z_{i},(i, j)\right\} \in E\left(R_{r}^{k}\right)$ for every $j \in[k]$. Then, given a $k$-equitable integer partition $\left\{m_{i, j}\right\}_{i \in[r], j \in[k]}$ of $n$ with $n /(10 \mathrm{kr}) \leq m_{i, j} \leq 10 \mathrm{n} /(\mathrm{kr})$ for every $i \in[r]$ and $j \in[k]$, there exists a mapping $f: V(H) \rightarrow[r] \times[k]$ and a set of special vertices $X \subseteq V(H)$ such that we have for every $i \in[r]$ and $j \in[k]$

(H1) $m_{i, j}-\xi_{n} \leq\left|f^{-1}(i, j)\right| \leq m_{i, j}+\xi_{n}$,

(H2) $|X| \leq \xi n$,

(H3) $\{f(x), f(y)\} \in E\left(R_{r}^{k}\right)$ for every $\{x, y\} \in E(H)$,

(H4) $y, z \in \cup_{j^{\prime} \in[k]} f^{-1}\left(i, j^{\prime}\right)$ for every $x \in f^{-1}(i, j) \backslash X$ and $x y, y z \in E(H)$,

(H5) $f(x)=(1, \sigma(x))$ for every $x$ in the first $\sqrt{\beta} n$ vertices of $\mathcal{L}$, and

(H6) $\left|\left\{x \in f^{-1}(i, j): \operatorname{deg}(x) \leq 2 D\right\}\right| \geq \frac{1}{24 D}\left|f^{-1}(i, j)\right|$.

Lemma 27 is a strengthened version of [13, Lemma 8]. The proof of [13, Lemma 8] is deterministic; here we use a probabilistic argument to show the existence of a function $f$ that also satisfies the additional property $(H 6)$, which is required for Theorem 8 . However, we still borrow ideas from the proof of [13, Lemma 8]. The proof of Lemma 27 will be given in Section 5.

During the pre-embeding, we embed a vertex $x$ of $H$ onto a vertex $v$ of $V_{0}$, and we also embed its neighbours $N_{H}(x)$. This creates restrictions on the vertices of $G$ to which we can embed the second neighbours, and for application of Lemma 16 we need certain conditions to be satisfied. The next lemma states that we can find vertices in $N_{G}(v)$, to which we will embed $N_{H}(x)$, satisfying these conditions.

Lemma 28 (Common neighbourhood lemma). For each $d>0, k \geq 2$, and $\Delta \geq 2$ there exists $\alpha>0$ such that for every $\varepsilon^{*} \in(0,1)$ there exists $\varepsilon_{0}>0$ such that for every $r \geq 1$ and every $0<\varepsilon \leq \varepsilon_{0}$ there exists $C^{*}>0$ such that the following is true. If $p \geq C^{*}(\log n / n)^{1 / \Delta}$, then $\Gamma=G(n, p)$ a.a.s. satisfies the 
The BANDWIDTh TheOREM IN SPARSE GRAPHS

following. Let $G=(V, E)$ be a (not necessarily spanning) subgraph of $\Gamma$ and $\left\{V_{i}\right\}_{i \in[k]} \cup\{W\}$ a vertex partition of a subset of $V$ such that the following are true for every $i, i^{\prime} \in[k]$.

(V1) $\frac{n}{4 k r} \leq\left|V_{i}\right| \leq \frac{4 n}{k r}$,

(V2) $\left(V_{i}, V_{i^{\prime}}\right)$ is $(\varepsilon, d, p)_{G^{-}}$lower-regular,

(V3) $|W| \geq 10^{-10} \frac{\varepsilon^{4} p n}{k^{4} r^{4}}$, and

(V4) $\left|N_{G}\left(w, V_{i}\right)\right| \geq d p\left|V_{i}\right|$ for every $w \in W$.

Then there exists a tuple $\left(w_{1}, \ldots, w_{\Delta}\right) \in\left(\begin{array}{c}W \\ \Delta\end{array}\right)$ such that for every $\Lambda, \Lambda^{*} \subseteq[\Delta]$, and every $i \neq i^{\prime} \in[k]$ we have

(W1) $\left|\bigcap_{j \in \Lambda} N_{G}\left(w_{j}, V_{i}\right)\right| \geq \alpha p^{|\Lambda|}\left|V_{i}\right|$,

(W2) $\left|\bigcap_{j \in \Lambda} N_{\Gamma}\left(w_{j}\right)\right| \leq\left(1+\varepsilon^{*}\right) p^{|\Lambda|} n$,

(W3) $\left|\bigcap_{j \in \Lambda} N_{\Gamma}\left(w_{j}, V_{i}\right)\right|=\left(1 \pm \varepsilon^{*}\right) p^{|\Lambda|}\left|V_{i}\right|$, and

(W4) $\left(\bigcap_{j \in \Lambda} N_{\Gamma}\left(w_{j}, V_{i}\right), \bigcap_{j^{*} \in \Lambda^{*}} N_{\Gamma}\left(w_{j^{*}}, V_{i^{\prime}}\right)\right)$ is $\left(\varepsilon^{*}, d, p\right)_{G^{-}}$lower-regular if $|\Lambda|,\left|\Lambda^{*}\right|<\Delta$ and either $\Lambda \cap$ $\Lambda^{*}=\varnothing$ or $\Delta \geq 3$ or both.

Let $H^{\prime}$ and $G^{\prime}$ denote the subgraphs of $H$ and $G$ that result from removing all vertices that were used in the pre-embedding process. As a last step before finally applying the sparse blow-up lemma, the clusters in $\left.\mathcal{V}\right|_{G^{\prime}}$ need to be adjusted to the sizes of $\left.W_{i, j}\right|_{H^{\prime}}$. The next lemma states that this is possible, and that after this redistribution the regularity properties needed for Lemma 16 still hold.

Lemma 29 (Balancing lemma). For all integers $k \geq 1, r_{1}, \Delta \geq 1$, and reals $\gamma, d>0$ and $0<\varepsilon<$ $\min \{d, 1 /(2 k)\}$ there exist $\xi>0$ and $C^{*}>0$ such that the following is true for every $p \geq C^{*}(\log n / n)^{1 / 2}$ and every $10 \gamma^{-1} \leq r \leq r_{1}$ provided that $n$ is large enough. Let $\Gamma$ be a graph on the vertex set $[n]$ and let $G=(V, E) \subseteq \Gamma$ be a (not necessarily spanning) subgraph with vertex partition $\mathcal{V}=\left\{V_{i, j}\right\}_{i \in[r], j \in[k]}$ that satisfies $n /(8 k r) \leq\left|V_{i, j}\right| \leq 4 n /(k r)$ for each $i \in[r], j \in[k]$. Let $\left\{n_{i, j}\right\}_{i \in[r], j \in[k]}$ be an integer partition of $\sum_{i \in[r], j \in[k]}\left|V_{i, j}\right|$. Let $R_{r}^{k}$ be a graph on the vertex set $[r] \times[k]$ with minimum degree $\delta\left(R_{r}^{k}\right) \geq((k-1) / k+$ $\gamma / 2) k r$ such that $K_{r}^{k} \subseteq B_{r}^{k} \subseteq R_{r}^{k}$. Suppose that the partition $\mathcal{V}$ satisfies the following properties for each $i \in[r]$, each $j \neq j^{\prime} \in[k]$, and each $v \in V$.

(B1) We have $n_{i, j}-\xi_{n} \leq\left|V_{i, j}\right| \leq n_{i, j}+\xi_{n}$,

(B2) $\mathcal{V}$ is $\left(\frac{\varepsilon}{4}, d, p\right)_{G}$-lower-regular on $R_{r}^{k}$ and $\left(\frac{\varepsilon}{4}, d, p\right)_{G}$-super-regular on $K_{r}^{k}$,

(B3) both $\left(N_{\Gamma}\left(v, V_{i, j}\right), V_{i, j^{\prime}}\right)$ and $\left(N_{\Gamma}\left(v, V_{i, j}\right), N_{\Gamma}\left(v, V_{i, j^{\prime}}\right)\right)$ are $\left(\frac{\varepsilon}{4}, d, p\right)_{G}$-lower-regular pairs, and

(B4) we have $\left|N_{\Gamma}\left(v, V_{i, j}\right)\right|=\left(1 \pm \frac{\varepsilon}{4}\right) p\left|V_{i, j}\right|$.

Then, there exists a partition $\mathcal{V}^{\prime}=\left\{V_{i, j}^{\prime}\right\}_{i \in[r], j \in[k]}$ of $V$ such that the following properties hold for each $i \in[r]$, each $j \neq j^{\prime} \in[k]$, and each $v \in V$. 
(B 1') We have $\left|V_{i, j}^{\prime}\right|=n_{i, j}$,

(B2') We have $\left|V_{i, j} \triangle V_{i, j}^{\prime}\right| \leq 10^{-10} \varepsilon^{4} k^{-2} r_{1}^{-2} n$,

$\left(B 3^{\prime}\right) V^{\prime}$ is $(\varepsilon, d, p)_{G}$-lower-regular on $R_{r}^{k}$ and $(\varepsilon, d, p)_{G}$-super-regular on $K_{r}^{k}$,

$\left(B 4^{\prime}\right)$ both $\left(N_{\Gamma}\left(v, V_{i, j}^{\prime}\right), V_{i, j^{\prime}}^{\prime}\right)$ and $\left(N_{\Gamma}\left(v, V_{i, j}^{\prime}\right), N_{\Gamma}\left(v, V_{i, j^{\prime}}^{\prime}\right)\right)$ are $(\varepsilon, d, p)_{G}$-lower-regular pairs, and

(B 5') For each $1 \leq s \leq \Delta$ and vertices $v_{1}, \ldots, v_{s} \in[n]$ we have

$$
\left|N_{\Gamma}\left(v_{1}, \ldots, v_{s} ; V_{i, j}\right) \triangle N_{\Gamma}\left(v_{1}, \ldots, v_{s} ; V_{i, j}^{\prime}\right)\right| \leq 10^{-10} \varepsilon^{4} k^{-2} r_{1}^{-2} \operatorname{deg}_{\Gamma}\left(v_{1}, \ldots, v_{s}\right)+C^{*} \log n .
$$

Furthermore, if for any two disjoint vertex sets $A, A^{\prime} \subseteq V(\Gamma)$ with $|A|,\left|A^{\prime}\right| \geq \frac{1}{50000 k r_{1}} \varepsilon^{2} \xi$ pn we have $e_{\Gamma}\left(A, A^{\prime}\right) \leq\left(1+\frac{1}{100} \varepsilon^{2} \xi\right) p|A|\left|A^{\prime}\right|$, and if 'lower-regular' is replaced with 'regular' in (B2), and (B 3), then we can replace 'lower-regular' with 'regular' in (B 3') and (B 4').

\section{The lemma for $G$}

In this section we prove the Lemma for $G$ (Lemma 26), which borrows from the proof of [12, Proposition 17] and from the proof of [10, Lemma 9]. Our strategy is as follows. We first apply Lemma 13 to obtain an equitable partition of $V(G)$ within whose reduced graph we can find a backbone graph by Theorem 1 . We let $Z_{1}$ be the vertices whose $\Gamma$-degrees are 'wrong' to this partition, or whose neighbourhoods fail to inherit lower-regularity (plus a few extra to maintain $k$-equitability), and we remove the vertices $Z_{1}$. Now there may be some vertices in each cluster which destroy super-regularity on the clique factor of the backbone graph. We redistribute these, and the exceptional set of the regular partition, to other clusters. Now we would like to say we are finished, but the moving of vertices may have destroyed some of the regularity inheritance, $\Gamma$-neighbourhood, and super-regularity properties we tried to obtain. However, it is easy to check that a vertex only witnesses failure of these properties if exceptionally many of its $\Gamma$-neighbours were moved from or to a cluster. We let $Z_{2}$ be the set of all such vertices, and remove them. We will see that $Z_{2}$ is so small that its removal does not significantly affect the properties we want, so that we can set $V_{0}=Z_{1} \cup Z_{2}$ and we are done.

Proof of Lemma 26. We first fix the constants in the proof. Given $\gamma>0, k \geq 2$ and $r_{0} \geq 1$, set $d=\frac{\gamma}{32}$. Let $\beta$ and $n_{0}$ be returned by Theorem 1 for input $\frac{1}{2} \gamma, 3 k$ and $k$. Let $r_{0}^{\prime}=\max \left\{n_{0}, k / d, 10 k / \beta, r_{0}\right\}$.

Given $\varepsilon \in\left(0, \frac{1}{2 k}\right]$, let $0<\varepsilon^{*} \leq 10^{-10} \varepsilon^{2} \gamma k^{-2}$ be small enough for both Lemmas 17 and 18 for input $\frac{1}{2} \varepsilon$ and $d$. Let $C$ be large enough for these applications of Lemmas 17 and 18, and also large enough for Proposition 19 with input $\frac{1}{1000}\left(\frac{\varepsilon^{*}}{k}\right)^{2}$.

Now Lemma 13, with input $\frac{1}{k} \varepsilon^{*}, \frac{k-1}{k}+\gamma$ and $r_{0}^{\prime}+k$, returns $r_{1}$. We set $C^{*}=1000 k^{3} r_{1}^{5} C /\left(\varepsilon^{*}\right)^{2}$.

Given $p \geq C^{*}\left(\frac{\log n}{n}\right)^{1 / 2}$, the random graph $G(n, p)$ a.a.s. satisfies the good events of Lemmas 17 and 18, and Proposition 19. We condition on $\Gamma=G(n, p)$ satisfying these good events.

Given $G \subseteq \Gamma$ with $\delta(G) \geq\left(\frac{k-1}{k}+\gamma\right) p n$, we apply Lemma 13, with input $\frac{1}{k} \varepsilon^{*}, \frac{k-1}{k}+\gamma, r_{0}^{\prime}+k$, and $d$, to $G$. We may do this because $G$ is a subgraph of $\Gamma$, and by choice of $C^{*}$ we have $C p^{-1} \log n \leq \frac{\varepsilon^{*} n}{k r_{1}}$, so that the condition of Lemma 13 is satisfied because the good event of Proposition 19 holds for $\Gamma$. The result is 
a $\left(\frac{1}{k} \varepsilon^{*}, p\right)$-lower-regular partition of $V(G)$ into $t^{\prime} \in\left[r_{0}^{\prime}+k, r_{1}\right]$ equally sized clusters, with exceptional set of size at most $\frac{1}{k} \varepsilon^{*} n$, whose $\left(\varepsilon^{*}, d, p\right)$-reduced graph has minimum degree at least $\left(\frac{k-1}{k}+\gamma-d-\frac{1}{k} \varepsilon^{*}\right) t^{\prime}$. We remove at most $k-1$ of these clusters to the exceptional set, obtaining an $\left(\varepsilon^{*}, p\right)$-lower-regular partition $\mathcal{U}$ of $V(G)$ into $k r$ equally sized clusters, where $r_{0}^{\prime} \leq k r \leq r_{1}$, with exceptional set $U_{0}$ of size at most $\varepsilon^{*} n$, whose $\left(\varepsilon^{*}, d, p\right)$-reduced graph $R_{r}^{k}$ has minimum degree at least $\left(\frac{k-1}{k}+\gamma-d-\frac{1}{k} \varepsilon^{*}\right) k r-k$.

By choice of $d$ and $\varepsilon^{*}$, and by choice of $r_{0}^{\prime}$, we have

$$
\left(\frac{k-1}{k}+\gamma-d-\frac{1}{k} \varepsilon^{*}\right) k r-k \geq\left(\frac{k-1}{k}+\frac{\gamma}{2}\right) k r .
$$

Observe that $B_{r}^{k}$ has bandwidth at most $2 k<\beta r_{0}^{\prime}$, and maximum degree less than $3 k$. Thus Theorem 1 , with input $\frac{\gamma}{2}, 3 k$, and $k$, in particular states that $R_{r}^{k}$ contains a copy of $B_{r}^{k}$. We fix one such copy. We let its vertices $\{(i, j)\}_{i \in[r], j \in[k]}$ label the vertices of $R_{r}^{k}$, and similarly let the cluster of $\mathcal{U}$ corresponding to the vertex $(i, j)$ of $B_{r}^{k}$ be $U_{i, j}$ for each $i \in[r]$ and $j \in[k]$. The partition $\mathcal{U}$ is equitable, and thus in particular $k$-equitable.

We now create $Z_{1}$ as follows. We start with all vertices $v$ of $G$ for which there are $(i, j)$ and $\left(i^{\prime}, j^{\prime}\right)$ in $V\left(R_{r}^{k}\right)$, with $\left\{(i, j),\left(i^{\prime}, j^{\prime}\right)\right\}$ an edge of $R_{r}^{k}$, such that either $\left(N_{\Gamma}\left(v, U_{i, j}\right), U_{i^{\prime}, j^{\prime}}\right)$ or $\left(N_{\Gamma}\left(v, U_{i, j}\right), N_{\Gamma}\left(v, U_{i^{\prime}, j^{\prime}}\right)\right)$ is not $\left(\frac{1}{2} \varepsilon, d, p\right)_{G}$-lower-regular. We add all vertices $v$ of $G$ for which there exists $U_{i, j}$ with $\operatorname{deg}_{\Gamma}\left(v, U_{i, j}\right) \neq$ $\left(1 \pm \varepsilon^{*}\right) p\left|U_{i, j}\right|$, or for which $\operatorname{deg}_{\Gamma}\left(v, U_{0}\right)>2 \varepsilon^{*} p n$. Finally we add a minimum number of vertices to obtain $k$-equitability of the sets $\left\{U_{i, j} \backslash Z_{1}\right\}_{i \in[r], j \in[k]}$. Note that we have $\left|U_{i, j}\right| \geq n /\left(2 k r_{1}\right)$ for each $i, j$, and we can estimate the number of vertices with more than $2 \varepsilon^{*} p n$ neighbours in $U_{0}$ by considering a superset of $U_{0}$ of size $\varepsilon^{*} n$. It follows that for each $i, j$ we have $\log \left(e n /\left|U_{i, j}\right|\right), \log \left(e n /\left|U_{0}\right|\right) \leq \log \left(e k r_{1} / \varepsilon^{*}\right)$. By Lemma 17 and Lemma 18, and Proposition 19, we have

$$
\left|Z_{1}\right| \leq 4 k r_{1}^{2} C \max \left\{p^{-2}, p^{-1} \log \left(e k r_{1} / \varepsilon^{*}\right)\right\} \leq 8 k^{2} r_{1}^{3} C p^{-2} / \varepsilon^{*} \leq \frac{\varepsilon^{*}}{k r_{1}} n,
$$

where the factor $k$ accounts for vertices removed to maintain $k$-equitability.

We now try to obtain super-regularity on the copy of $K_{r}^{k}$ in $B_{r}^{k}$. For each $i \in[r]$ and $j \in[k]$ let $W_{i, j}$ be the vertices of $U_{i, j} \backslash Z_{1}$ which have less than $\left(d-2 \varepsilon^{*}\right) p\left|U_{i, j^{\prime}}\right|$ neighbours in $U_{i, j^{\prime}}$ for some $j^{\prime} \neq j$. Because $\left(U_{i, j}, U_{i, j^{\prime}}\right)$ is $\left(\varepsilon^{*}, d, p\right)$-lower-regular for each $i \in[r]$ and $j \neq j^{\prime} \in[k]$, we have $\left|W_{i, j}\right| \leq k \varepsilon^{*}\left|U_{i, j}\right|$ for each $i \in[r]$ and $j \in[k]$.

Now let $W$ contain $U_{0} \backslash Z_{1}$ together with all $W_{i, j}$ for $i \in[r]$ and $j \in[k]$, and a minimum number of additional vertices from $V(G) \backslash Z_{1}$ to obtain $k$-equitability of the sets $\left\{U_{i, j} \backslash\left(Z_{1} \cup W\right)\right\}_{i \in[r], j \in[k]}$. By construction, we have $|W| \leq \varepsilon^{*} n+k r \cdot k \varepsilon^{*} \frac{n}{k r} \leq 2 k \varepsilon^{*} n$.

Given any $w \in W$, because $w \notin Z_{1}$ we have

$$
\operatorname{deg}_{\Gamma}\left(w, U_{0}\right) \leq 2 \varepsilon^{*} p n \quad \text { and } \quad \operatorname{deg}_{\Gamma}\left(w, U_{i, j}\right) \leq\left(1+\varepsilon^{*}\right) p\left|U_{i, j}\right|
$$

for each $i \in[r]$ and $j \in[k]$. Now let us consider the edges of $G$ leaving $w$. At most $2 \varepsilon^{*} p n$ of these go to $U_{0}$, and by definition at most $2 d p n$ go to sets $U_{i, j}$ such that $\operatorname{deg}_{G}\left(w, U_{i, j}\right) \leq 2 d p\left|U_{i, j}\right|$. Since $\operatorname{deg}_{G}(w) \geq$ $\left(\frac{k-1}{k}+\gamma\right) p n$, at least $\left(\frac{k-1}{k}+\frac{\gamma}{2}\right) p n$ edges leaving $w$ go to sets $U_{i, j}$ with $\operatorname{deg}_{G}\left(w, U_{i, j}\right) \geq 2 d p\left|U_{i, j}\right|$. Since $\left|U_{i, j}\right| \leq \frac{1}{k r} n$, in particular there are at least

$$
\frac{\left(\frac{k-1}{k}+\frac{\gamma}{2}\right) p n}{\left(1+\varepsilon^{*}\right) p \frac{n}{k r}} \geq\left(\frac{k-1}{k}+\frac{\gamma}{4}\right) k r
$$


sets $U_{i, j}$ with $i \in[r]$ and $j \in[k]$ such that $\operatorname{deg}_{G}\left(w, U_{i, j}\right) \geq 2 d p\left|U_{i, j}\right|$. It follows that there are at least $\frac{\gamma}{4} r$ indices $i \in[r]$ such that $\operatorname{deg}_{G}\left(w, U_{i, j}\right) \geq 2 d p\left|U_{i, j}\right|$ for each $j \in[k]$.

We now assign to each $w \in W$ sequentially an index $c(w) \in[r] \times[k]$. For each $w$, we choose $c(w)=(i, j)$ as follows. The index $i$ is chosen minimal in $[r]$ such that $\operatorname{deg}_{G}\left(w, U_{i, j^{\prime}}\right) \geq 2 d p\left|U_{i, j^{\prime}}\right|$ for each $j^{\prime} \in[k]$, but at most $\frac{100}{r} k \varepsilon^{*} \gamma^{-1} n$ vertices $w^{\prime} \in W$ have so far been assigned $c\left(w^{\prime}\right)=\left(i, j^{\prime}\right)$ for any $j^{\prime} \in[k]$. We choose $j \in[k]$ minimising the number of vertices $w^{\prime} \in W$ with $c(w)=(i, j)$. Because $|W| \leq 2 k \varepsilon^{*} n$, this assignment is always possible.

Next, for each $i \in[r]$ and $j \in[k]$, we let $V_{i, j}^{\prime}$ consist of $U_{i, j} \backslash\left(Z_{1} \cup W_{i, j}\right)$, together with all $w \in W$ such that $c(w)=(i, j)$. By construction, we have

$$
\left|U_{i, j} \triangle V_{i, j}^{\prime}\right| \leq\left|Z_{1}\right|+\left|W_{i, j}\right|+\frac{100}{r} k \varepsilon^{*} \gamma^{-1} n \leq 1000 k^{2} \varepsilon^{*} \gamma^{-1}\left|U_{i, j}\right|
$$

Finally, we let $Z_{2}$ be the vertices $v \in V(G) \backslash Z_{1}$ with $\operatorname{deg}_{\Gamma}\left(v, U_{i, j} \triangle V_{i, j}^{\prime}\right) \geq 2000 k^{2} \varepsilon^{*} \gamma^{-1} p\left|U_{i, j}\right|$ for some $i \in[r]$ and $j \in[k]$, together with a minimum number of additional vertices of $V(G) \backslash Z_{1}$ to obtain $k$-equitability of the sets $V_{i, j}:=V_{i, j}^{\prime} \backslash Z_{2}$. We set $V_{0}=Z_{1} \cup Z_{2}$. We claim that $\mathcal{V}=\left\{V_{i, j}\right\}_{i \in[r], j \in[k]}$ is the desired partition of $V(G) \backslash V_{0}$.

Note that the sets $V_{i, j}^{\prime}$ and $V_{i, j^{\prime}}^{\prime}$ differ in size by at most one for any $i \in[r]$ and $j, j^{\prime} \in[k]$, by our construction of the assignment $c$. We apply Proposition 19 to estimate the number of vertices $v \in$ $V(G) \backslash Z_{1}$ with $\operatorname{deg}_{\Gamma}\left(v, U_{i, j} \triangle V_{i, j}^{\prime}\right) \geq 2000 k^{2} \varepsilon^{*} \gamma^{-1} p\left|U_{i, j}\right|$ by considering a superset of $U_{i, j} \triangle V_{i, j}^{\prime}$ of size $1000 k^{2} \varepsilon^{*} \gamma^{-1}\left|U_{i, j}\right| \geq \varepsilon^{*} n / r_{1}$. By Proposition 19 we thus have

$$
\left|Z_{2}\right| \leq r_{1}+C k r_{1} p^{-1} \log \left(e r_{1} / \varepsilon^{*}\right) \leq 4 C k r_{1}^{2} p^{-1} / \varepsilon^{*} \leq \frac{\varepsilon^{*}}{k r_{1}} p n
$$

This gives

$$
\left|U_{i, j} \triangle V_{i, j}\right| \leq\left|U_{i, j} \triangle V_{i, j}^{\prime}\right|+\left|Z_{2}\right| \leq 2000 k^{2} \varepsilon^{*} \gamma^{-1}\left|U_{i, j}\right|
$$

Now given any $v \in V(G) \backslash V_{0}$, for each $i \in[r]$ and $j \in[k]$, because $v \notin Z_{2}$ we have $\operatorname{deg}_{\Gamma}\left(v, U_{i, j} \triangle V_{i, j}^{\prime}\right) \leq$ $2000 k^{2} \varepsilon^{*} \gamma^{-1} p\left|U_{i, j}\right|$. We thus have

$$
\operatorname{deg}_{\Gamma}\left(v, U_{i, j} \triangle V_{i, j}\right) \leq 2000 k^{2} \varepsilon^{*} \gamma^{-1} p\left|U_{i, j}\right|+\left|Z_{2}\right| \leq 3000 k^{2} \varepsilon^{*} \gamma^{-1} p\left|U_{i, j}\right|,
$$

and because $v \notin Z_{1}$ we have $\operatorname{deg}_{\Gamma}\left(v, U_{i, j}\right)=\left(1 \pm \varepsilon^{*}\right) p\left|U_{i, j}\right|$, and hence by (4.3)

$$
\operatorname{deg}_{\Gamma}\left(v, V_{i, j}\right)=\left(1 \pm 10000 k^{2} \varepsilon^{*} \gamma^{-1}\right) p\left|V_{i, j}\right|
$$

Adding up (4.1) and (4.2), we conclude

$$
\left|V_{0}\right| \leq 8 k^{2} r_{1}^{3} C p^{-2} / \varepsilon^{*}+4 C k r_{1}^{2} p^{-1} / \varepsilon^{*} \leq C^{*} p^{-2},
$$

as desired. The partition $\mathcal{V}=\left\{V_{i, j}\right\}_{i \in[r], j \in[k]}$ is by construction $k$-equitable, and the graph $R_{r}^{k}$ has minimum degree $\left(\frac{k-1}{k}+\frac{\gamma}{2}\right) k r$ as desired.

For each $i \in[r]$ and $j \in[k]$ we have $\left|U_{i, j}\right|=\left(1 \pm \varepsilon^{*}\right) \frac{n}{k r}$, and so (4.3) and our choice of $\varepsilon^{*}$ give (G1).

Next, if $\left\{(i, j),\left(i^{\prime}, j^{\prime}\right)\right\}$ is an edge of $R_{r}^{k}$, then $G$ is $\left(\varepsilon^{*}, d, p\right)$-lower-regular on $\left(U_{i, j}, U_{i^{\prime}, j^{\prime}}\right)$ by construction. By (4.3), Proposition 12, and our choice of $\varepsilon^{*}, G$ is $(\varepsilon, d, p)$-lower-regular on $\left(V_{i, j}, V_{i^{\prime}, j^{\prime}}\right)$. Given 
$i \in[r]$ and $j \neq j^{\prime} \in[k]$, let $v$ be a vertex of $V_{i, j}$. Observe that since $v \in V_{i, j}$, either we have $v \in U_{i, j}$, in which case, since $v \notin W$ we have $\operatorname{deg}_{G}\left(v, U_{i, j^{\prime}}\right) \geq\left(d-2 \varepsilon^{*}\right) p\left|U_{i, j^{\prime}}\right|$, or $v$ is in $W$ and has $c(v)=(i, j)$, in which case $\operatorname{deg}_{G}\left(v, U_{i, j^{\prime}}\right) \geq d p\left|U_{i, j^{\prime}}\right|$. By (4.3) and (4.4) we have

$$
\operatorname{deg}_{G}\left(v, V_{i, j^{\prime}}\right) \geq\left(d-2 \varepsilon^{*}\right) p\left|U_{i, j^{\prime}}\right|-3000 k^{2} \varepsilon^{*} \gamma^{-1} p\left|U_{i, j^{\prime}}\right| \geq(d-\varepsilon) p\left|V_{i, j^{\prime}}\right|,
$$

giving $(G 2)$.

If $\left\{(i, j),\left(i^{\prime}, j^{\prime}\right)\right\} \in E\left(R_{r}^{k}\right)$, then for any $v \in V(G) \backslash V_{0}$, since $v \notin Z_{1}$, the pairs $\left(N_{\Gamma}\left(v, U_{i, j}\right), U_{i^{\prime}, j^{\prime}}\right)$ and $\left(N_{\Gamma}\left(v, U_{i, j}\right), N_{\Gamma}\left(v, U_{i^{\prime}, j^{\prime}}\right)\right)$ are $\left(\frac{1}{2} \varepsilon, d, p\right)_{G}$-lower-regular. Using (4.3) and (4.4), Proposition 12 and our choice of $\varepsilon^{*}$, we conclude $(G 3)$.

Finally, $(G 4)$ follows from (4.5) and our choice of $\varepsilon^{*}$.

Note that if we alter the definition of $Z_{1}$, removing the condition on $\left(N_{\Gamma}\left(v, U_{i, j}\right), N_{\Gamma}\left(v, U_{i^{\prime}, j^{\prime}}\right)\right)$, then we do not need to use Lemma 18 and the bound in (4.1) improves to $\left|Z_{1}\right| \leq 8 k^{2} r_{1}^{3} C p^{-1} / \varepsilon^{*}$. Thus, if we only require (G $\left.3^{\prime}\right)$, we obtain $\left|V_{0}\right| \leq C^{*} p^{-1}$ as claimed.

\section{The lemma for $H$}

In this section we present the proof of Lemma 27. The proof idea is as follows. First, given the zero-free labelling $\mathcal{L}$ and $(k+1)$-colouring $\sigma$ of $H$, we split $\mathcal{L}$ into the blocks of the definition of zero-freeness. We partition the blocks into $r$ 'sections' of consecutive blocks, such that the $i$-th section contains about $\sum_{j \in[k]} m_{i, j}$ vertices, and furthermore such that the 'boundary vertices, namely the first and last $\beta n$ vertices of each section, do not receive colour zero. Now it is easy to check that assigning the vertices of colour $j$ in the $i$-th section to $(i, j)$ for each $i \in[r]$ and $j \in[k]$, and the vertices of colour zero in the $i$-th section to $z_{i}$, is a graph homomorphism. However it can be very unbalanced, since different colours in $[k]$ may be used with very different frequencies in each section. To fix this, we replace $\sigma$ with a new colouring $\sigma^{\prime}$, which we obtain as follows. We partition each section into 'intervals' of consecutive blocks, and for each interval except the last in each section, we pick a random permutation of $[k]$. We will show that there is a colouring $\sigma^{\prime}$ such that all but the first few vertices of each interval are coloured according to the permutation applied to $\sigma$, with vertices of colour zero staying coloured zero. We use this colouring $\sigma^{\prime}$ in place of $\sigma$ to define the mapping $f$. We let $X$ consist of all vertices whose distance is two or less to either boundary vertices, vertices near the start of an interval, or colour zero vertices.

To complete the proof, we show that so few vertices receive colour zero that they do not much affect the desired conclusions. Now the mapping $f$ is in expectation balanced, and using Lemma 23 we can show that it is also with high probability close to balanced. It is also easy to check that, since $H$ is $D$-degenerate, in the $i$-th section of $\mathcal{L}$ there are many vertices of degree at most $2 D$. In expectation these are distributed about evenly over the $\{(i, j)\}_{j \in[k]}$ by $f$, and again McDiarmid's inequality shows that with high probability the same holds. These two observations give us $(H 1)$ and $(H 6)$, while the other four desired conclusions hold by construction.

Proof of Lemma 27. For given $D \geq 1$, set $\alpha=1 /(24 D)$. Let $k, r \geq 1$ and $\xi, \beta>0$ be given, where $\xi \leq 1 /(k r)$ and $\beta \leq 10^{-10} \xi^{2} /\left(D k^{4} r\right)$. Let $H$ and $K_{r}^{k} \subseteq B_{r}^{k} \subseteq R_{r}^{k}$ be graphs as in the statement of the lemma. Let $\mathcal{L}$ be the given labelling of $V(H)$ of bandwidth at most $\beta n$. We denote the set of the first $\sqrt{\beta} n$ vertices of $\mathcal{L}$ by $F$. Let $\sigma: V(H) \rightarrow\{0, \ldots k\}$ be the given proper $(k+1)$-colouring of $V(H)$ that is 
$(10 / \xi, \beta)$-zero-free with respect to $\mathcal{L}$ and such that $\sigma(F) \subseteq[k]$. Also, let $z_{1}, \ldots, z_{n}$ be vertices such that $z_{i} \in([r] \backslash\{i\}) \times[k]$ with $\left\{z_{i},(i, j)\right\} \in E\left(R_{r}^{k}\right)$ for every $i \in[r]$ and $j \in[k]$. Finally, set $b=k / \sqrt{\beta}$.

Let $\left\{m_{i, j}\right\}_{i \in[r], j \in[k]}$ be the given $k$-equitable integer partition of $n$ with $n /(10 k r) \leq m_{i, j} \leq 10 n /(k r)$ for every $i \in[r]$ and $j \in[k]$.

Let us now introduce the notation that we use in this proof. Recall that for every $t \in[1 /(4 k \beta)]$ the $i$-th block is defined as

$$
B_{t}:=\{(t-1) 4 k \beta n+1, \ldots, t 4 k \beta n\} .
$$

Next we split the labelling $\mathcal{L}$ into $r$ sections, where the first and the last block of each section are zero-free. Each section is partitioned into intervals, each of which but possibly the last one consists of $b$ blocks.

Since $\sigma$ is $(10 / \xi, \beta)$-zero-free with respect to $\mathcal{L}$, we can choose indices $0=t_{0} \leq t_{1} \leq \ldots \leq t_{r-1} \leq$ $t_{r}=1 /(4 k \beta)$ such that $B_{t_{i}}$ and $B_{t_{i}+1}$ are zero-free blocks for every $i \in[r]$ and

$$
\sum_{t=1}^{t_{i}}\left|B_{t}\right| \leq \sum_{t=1}^{i} \sum_{j \in[k]} m_{t, j}<12 k \beta n+\sum_{t=1}^{t_{i}}\left|B_{t}\right| .
$$

Since $m_{i, j} \geq n /(10 k r)>12 k \beta n$, indices $t_{0}, \ldots, t_{r}$ are distinct. For every $i \in[r]$ we define the $i$-th section $S_{i}$ as

$$
\bigcup_{t=t_{i-1}+1}^{t_{i}} B_{t}
$$

This means by the choice of the indices $t_{0}, \ldots, t_{r}$ that the first and last block of each section are zero-free. Since $\left\{m_{i, j}\right\}_{i \in[r], j \in[k]}$ is a $k$-equitable partition, we have in particular

$$
\frac{1}{k}\left(\left|S_{i}\right|-12 k \beta n\right) \leq m_{i, j} \leq \frac{1}{k}\left(\left|S_{i}\right|+12 k \beta n\right) .
$$

The last $\beta n$ vertices of the blocks $B_{t_{i}}$ and the first $\beta n$ vertices of the blocks $B_{t_{i+1}}$ are called boundary vertices of $H$. Notice that colour zero is never assigned to boundary vertices by $\sigma$. For each $i \in[r]$, we split $S_{i}$ into $s_{i}:=\left\lceil\left(t_{i}-t_{i-1}-1\right) / b\right\rceil$ intervals, where each of the first $\left(s_{i}-1\right)$ intervals is the concatenation of exactly $b$ blocks and the last interval consists of $t_{i}-t_{i-1}-1-b\left(s_{i}-1\right) \leq b$ blocks. Therefore, for every $i \in[r]$, we have

$$
s_{i}(b-1) 4 k \beta n+1 \leq\left|S_{i}\right| \leq s_{i} b 4 k \beta n .
$$

Using Equation (5.1), $b=k / \sqrt{\beta}$, and $n /(10 k r) \leq m_{i, j} \leq 10 n /(k r)$ we get, for every $i \in[r]$, the following bounds on $s_{i}$

$$
\frac{1}{100 r k^{2} \sqrt{\beta}} \leq s_{i} \leq \frac{10}{r k^{2} \sqrt{\beta}} .
$$

We denote the intervals of the $i$-th section by $I_{i, 1}, \ldots, I_{i, s_{i}}$. Let $B_{i, \ell}^{\mathrm{sw}}$ denote the union of the first two blocks of each interval $I_{i, \ell}$. All of these blocks but $B_{i, 1}^{\mathrm{sw}}$ and $B_{i, s_{i}}^{\mathrm{sw}}$ will be used to switch colours within parts of $H$. Notice that we have $\left|B_{i, \ell}^{\mathrm{sw}}\right|=8 \mathrm{k} \beta n$ and, since $\sigma$ is $(10 / \xi, \beta)$-zero-free with respect to $\mathcal{L}$, at least one of the two blocks of $B_{i, \ell}^{\mathrm{sw}}$ is zero-free. We will not use $B_{i, 1}^{\mathrm{sw}}$ and $B_{i, s_{i}}^{\mathrm{sw}}$ to switch colours because we will need that the boundary vertices do not receive colour zero.

For every $i \in[r]$ and every $\ell \in\left\{2, \ldots, s_{i}-1\right\}$, we choose a permutation $\pi_{i, \ell}:[k] \rightarrow[k]$ uniformly at random. 
The next claim ensures that we can use zero-free blocks to obtain a proper colouring of the vertex set such that vertices before the switching block are coloured according to the original colouring and the colours of the vertices after the switching block are permuted as wished. A proof can be found in [13].

Claim 30 ([13]). Let $\sigma:[n] \rightarrow\{0, \ldots, k\}$ be a proper $(k+1)$-colouring of $H$, let $B_{t}$ be a zero-free block and let $\pi$ be any permutation of $[k]$. Then there exists a proper $(k+1)$-colouring $\sigma^{\prime}$ of $H$ with $\sigma^{\prime}(x)=\sigma(x)$ for all $x \in \bigcup_{i<t} B_{i}$ and

$$
\sigma^{\prime}(x)= \begin{cases}\pi(\sigma(x)) & \text { if } \sigma(x) \neq 0 \\ 0 & \text { otherwise }\end{cases}
$$

for all $x \in \bigcup_{i>t} B_{i}$.

We use Claim 30 to switch colours at the beginning of each interval except for the first and last interval of each section. More precisely, we switch colours within the sets $B_{i, \ell}^{\mathrm{sw}}$ so that the colouring of the remaining vertices in the interval $I_{i, \ell}$ matches $\pi_{i, \ell}$. Note that we can indeed use $B_{i, \ell}^{\mathrm{sW}}$ to do the switching since one of the two blocks in $B_{i, \ell}^{\mathrm{sw}}$ is zero-free. In particular, we get a proper $(k+1)$-colouring $\sigma^{\prime}=\sigma^{\prime}\left(\pi_{1,2}, \ldots, \pi_{r, s_{r}-1}\right): V(H) \rightarrow\{0, \ldots k+1\}$ of $H$ that fulfils the following. For every $x \in I_{1,1}$ we have

$$
\sigma^{\prime}(x)=\sigma(x),
$$

for each $i \in[r]$ and $\ell \in\left\{2, \ldots, s_{i}-1\right\}$ and every $x \in I_{i, \ell} \backslash B_{i, \ell}^{\mathrm{sw}}$ we have that

$$
\sigma^{\prime}(x)= \begin{cases}\pi_{i, \ell}(\sigma(x)) & \text { if } \sigma(x) \neq 0 \\ 0 & \text { otherwise }\end{cases}
$$

and for each $i \in[r]$ and every $x \in I_{i, s_{i}} \cup I_{i+1,1}$ (where $I_{r+1,1}:=\varnothing$ ) we have that

$$
\sigma^{\prime}(x)=\pi_{i, s_{i}-1}(\sigma(x)) .
$$

While $\sigma^{\prime}$ is well-defined on the sets $B_{1,2}^{\mathrm{sw}}, \ldots, B_{r, s_{r}-1}^{\mathrm{sw}}$ by Claim 30 , the definition on these sets is rather complicated as it is depends on which of the two blocks in $B_{i, \ell}^{\mathrm{sw}}$ is zero-free and on the colourings before and after the switching. However, the precise definition on these sets is not important for the remainder of the proof. Hence, we omit it here. Observe that $\sigma^{\prime}$ never assigns colour zero to boundary vertices.

Using $\sigma^{\prime}$ we now define $f=f\left(\pi_{1,2}, \ldots, \pi_{r, s_{r}-1}\right): V(H) \rightarrow[r] \times[k]$ as follows. For each $i \in[r]$ and $x \in S_{i}$ we set

$$
f(x):= \begin{cases}\left(i, \sigma^{\prime}(x)\right) & \text { if } \sigma^{\prime}(x) \neq 0 \\ z_{i} & \text { otherwise }\end{cases}
$$

where $z_{i} \in([r] \backslash\{i\}) \times[k]$ is the vertex defined in the statement of the lemma. Let $X$ consist of all vertices at distance two or less from a boundary vertex of $\mathcal{L}$, from a vertex in any $B_{i, \ell}^{\mathrm{sw}}$, or from a colour zero vertex. We now show that $f$ and $X$ satisfy Properties (H2)-(H 5) with probability 1 and Properties (H 1$)$ and (H6) with high probability. In particular, this implies that the desired $f$ and $X$ exist. 
We start with Property $(H 1)$. For each $i \in[r]$ let

$$
S_{i}^{*}:=S_{i} \backslash\left(\bigcup_{\ell \in\left[s_{i}\right]} B_{i, \ell}^{\mathrm{sW}} \cup I_{i, 1} \cup I_{i, s_{i}}\right)
$$

be the set of all vertices in $S_{i}$ except for the first and last interval and the first two blocks of each interval of $S_{i}$. We will also make use of the following restricted function

$$
f^{*}=f^{*}\left(\pi_{1,2}, \ldots, \pi_{r, S_{r}}\right):=\left.f\right|_{\bigcup_{i \in[r]} S_{i}^{*}}
$$

The basic idea of the proof of Property $(H 1)$ is to determine bounds on $\left|f^{*-1}(i, j)\right|$ that hold with positive probability and then deduce the desired bounds on $\left|f^{-1}(i, j)\right|$. Since the permutations $\pi_{i, \ell}$ were chosen uniformly at random, we have by definition of $f^{*}$ that the expected number of vertices mapped to $(i, j) \in[r] \times[k]$ by $f^{*}$ is

$$
\begin{aligned}
\mathbb{E}\left[\left|f^{*-1}(i, j)\right|\right]=\frac{1}{k}\left[\left(s_{i}-2\right)(b-2) 4 k \beta n-\mid\left\{x \in S_{i}^{*}:\right.\right. & \sigma(x)=0\} \mid] \\
& +\mid \bigcup_{l \in[r] \backslash\{i\}}\left\{x \in S_{l}^{*}: \sigma(x)=0 \text { and } z_{l}=(i, j)\right\} \mid .
\end{aligned}
$$

In particular, the following bounds on the expected value of $\left|f^{*-1}(i, j)\right|$ hold.

$$
\mathbb{E}\left[\left|f^{*-1}(i, j)\right|\right] \leq\left(s_{i}-2\right)(b-2) 4 \beta n+\frac{\xi}{10} n
$$

and

$$
\mathbb{E}\left[\left|f^{*-1}(i, j)\right|\right] \geq(1-\xi / 10)\left(s_{i}-2\right)(b-2) 4 \beta n \geq\left(s_{i}-2\right)(b-2) 4 \beta n-\frac{\xi}{10} n .
$$

If one replaced a permutation $\pi_{i, \ell}$ by some other permutation $\tilde{\pi}:[k] \rightarrow[k]$, then $\left|f^{*-1}(i, j)\right|$ would change by at most $(b-2) 4 k \beta n$. Hence, by McDiarmid's Inequality (Lemma 23) we have

$$
\begin{aligned}
\mathbb{P}\left[\left|\left(s_{i}-2\right)(b-2) 4 \beta n-\right| f^{*-1}(i, j)|| \geq \frac{\xi}{5} n\right] & \stackrel{(5.3),(5.4)}{\leq} \\
& \mathbb{P}\left[\left|\mathbb{E}\left[\mid f^{*-1}(i, j)\right]-\right| f^{*-1}(i, j)|| \geq \frac{\xi}{10} n\right] \leq 2 \exp \left\{-\frac{\xi^{2} n^{2}}{50\left(s_{i}-2\right)((b-2) 4 k \beta n)^{2}}\right\}
\end{aligned}
$$

Taking the union bound over all $j \in[k]$ and using $s_{i} \leq 10 /\left(r k^{2} \sqrt{\beta}\right)$ and $b=k / \sqrt{\beta}$ as well as $\beta \leq 10^{-10} \xi^{2} /\left(D k^{4} r\right)$ yields

$$
\mathbb{P}\left[\left|\left(s_{i}-2\right)(b-2) 4 \beta n-\right| f^{*-1}(i, j)|| \geq \frac{\xi}{5} n \text { for all } j \in[k]\right] \leq 2 k \exp \left\{-\frac{\xi^{2} r}{8000 k^{2} \sqrt{\beta}}\right\} \leq 2 k e^{-k}<1 .
$$


Observe that $\left|f^{*-1}(i, j)\right|$ is independent of the choices for $\pi_{i^{\prime}, \ell}$ if $i^{\prime} \neq i$. Hence, with positive probability we have, for every $i \in[r]$ and $j \in[k]$, that

$$
\left(s_{i}-2\right)(b-2) 4 \beta n-\frac{\xi}{5} n \leq\left|f^{*-1}(i, j)\right| \leq\left(s_{i}-2\right)(b-2) 4 \beta n+\frac{\xi}{5} n .
$$

From the definition of $f^{*}$ it follows that $\left|f^{-1}(i, j)\right| \geq\left|f^{*-1}(i, j)\right|$ and

$$
\left|f^{-1}(i, j)\right| \leq\left|f^{*-1}(i, j)\right|+\left|I_{i, 1}\right|+\left|I_{i, s_{i}}\right|+\sum_{\ell=2}^{s_{i}-1}\left|B_{i, \ell}^{\mathrm{sw}}\right|+\mid\left\{x \in \bigcup_{\imath \in[r] \backslash\{i\}} S_{\imath} \backslash S_{\imath}^{*}: \sigma^{\prime}(x)=0 \text { and } z_{\imath}=(i, j)\right\} \mid .
$$

Using $s_{i} \leq 10 /\left(r k^{2} \sqrt{\beta}\right)$ and $b=k / \sqrt{\beta}$ and $\beta \leq 10^{-10} \xi^{2} /\left(D k^{4} r\right)$, with positive probability we have for every $i \in[r]$ and $j \in[k]$ that

$$
\begin{aligned}
\left|f^{-1}(i, j)\right| & \geq\left|f^{*-1}(i, j)\right| \geq\left(s_{i}-2\right)(b-2) 4 \beta n-\frac{\xi}{5} n \\
& \geq\left(s_{i}-2\right)(b-2) 4 \beta n-\frac{\xi}{5} n+\left(8\left(s_{i}+b\right) \beta n-\frac{4}{5} \xi_{n}\right) \\
& \geq s_{i} b 4 \beta n+16 \beta n-\xi n \\
& \stackrel{(5.2)}{\geq} \frac{1}{k}\left(\left|S_{i}\right|+16 k \beta n\right)-\xi n \stackrel{(5.1)}{\geq} m_{i, j}-\xi n .
\end{aligned}
$$

On the other hand,

$$
\begin{aligned}
\left|f^{-1}(i, j)\right| \leq & \left|f^{*-1}(i, j)\right|+\left|I_{i, 1}\right|+\left|I_{i, s_{i}}\right|+\sum_{\ell=2}^{s_{i}-1}\left|B_{i, \ell}^{\mathrm{sw}}\right| \\
& +\mid\left\{x \in \bigcup_{i \in[r] \backslash\{i\}} S_{\imath} \backslash S_{i}^{*}: \sigma^{\prime}(x)=0 \text { and } z_{l}=(i, j)\right\} \mid \\
\leq & \left(s_{i}-2\right)(b-2) 4 \beta n+\frac{\xi}{5} n+8 b k \beta n+\left(s_{i}-2\right) 8 k \beta n+\frac{\xi}{10} n \\
\leq & \frac{1}{k}\left(\left(s_{i}-2\right)(b-2) 4 k \beta n\right)+\xi n \\
\leq & \frac{1}{k}\left(\left|S_{i}\right|-12 k \beta n\right)+\xi n \stackrel{(5.1)}{\leq} m_{i, j}+\xi n,
\end{aligned}
$$

which shows that Property $(H 1)$ holds with positive probability.

By definition of $X$, since $\mathcal{L}$ is a $\beta n$-bandwidth ordering, any vertex in $X$ is at distance at most $2 \beta n$ in $\mathcal{L}$ from a boundary vertex, a vertex of some $B_{i, \ell}^{\mathrm{sw}}$, or from a vertex assigned colour zero. Because there are $r$ sections, the boundary vertices form $r-1$ intervals each of length $2 \beta n$, and so at most $6 r \beta n$ vertices of $H$ are at distance 2 or less from a boundary vertex. There are $\sum_{i \in[r]} s_{i}$ intervals and hence $\sum_{i \in[r]} s_{i}$ switching blocks each of size $8 k \beta n$. As $s_{i} \leq 10 /\left(r k^{2} \sqrt{\beta}\right)$ for every $i \in[r]$, there are at most $(4+8 k) \beta n \cdot 10 /\left(k^{2} \sqrt{\beta}\right)$ vertices at distance 2 or less from a vertex of some switching block. Similarly, because $\mathcal{L}$ is $(10 / \xi, \beta)$-zero-free, in any consecutive $10 / \xi$ blocks at most one contains vertices of colour 
zero, and hence at most $(8+4 k) \beta n$ vertices in any such $10 / \xi$ consecutive blocks are at distance 2 or less from a vertex of colour zero. Thus we have

$$
|X| \leq 6 r \beta n+(4+8 k) \beta n\left(\frac{10}{k^{2} \sqrt{\beta} n}\right)+(8+4 k) \beta n\left(\frac{n}{4 k \beta n \cdot 10 / \xi}+1\right) \leq 6 r \beta n+\frac{1}{4} \xi_{n}+\frac{1}{3} \xi_{n} \leq \xi_{n}
$$

which gives $(H 2)$.

Since $\sigma^{\prime}$ is a proper colouring, and boundary vertices are not adjacent to colour zero vertices, by definition, $f$ restricted to the boundary vertices is a graph homomorphism to $B_{r}^{k}$. On the other hand, on each section $S_{i}$, again since $\sigma^{\prime}$ is a proper colouring and since $\{(i, j)\}_{j \in[k]} \cup\left\{z_{i}\right\}$ forms a clique in $R_{r}^{k}, f$ is a graph homomorphism to $R_{r}^{k}$. Since $\mathcal{L}$ is a $\beta n$-bandwidth ordering, any edge of $H$ is either contained in a section or goes between two boundary vertices, and we conclude that $f$ is a graph homomorphism from $H$ to $R_{r}^{k}$, giving (H3).

Now, given $i \in[r]$ and $j \in[k]$, and $x \in f^{-1}(i, j) \backslash X$, if $\{x, y\}$ and $\{y, z\}$ are edges of $H$, then $y$ and $z$ are at distance two or less from $x$ in $H$. In particular, by definition of $X$ neither $y$ nor $z$ is either a boundary vertex, in any $B_{i, \ell}^{\mathrm{sw}}$, or assigned colour zero. Since boundary vertices appear in intervals of length $2 \beta n$ in $\mathcal{L}$, and $\mathcal{L}$ is a $\beta n$-bandwidth ordering, it follows that $y$ and $z$ are both in $S_{i}$. Furthermore, suppose $x \in I_{i, \ell}$ for some $\ell$. By definition $x \notin B_{i, \ell}^{\mathrm{sw}}$. Because $B_{i, \ell}^{\mathrm{sw}}$ and $B_{i, \ell+1}^{\mathrm{sw}}$ (if the latter exists) are intervals of length $8 k \beta n$, both $y$ and $z$ are also in $I_{i, \ell} \backslash B_{i, \ell}^{\mathrm{sw}}$, and in particular both $y$ and $z$ are in $\bigcup_{j^{\prime} \in[k]} f^{-1}\left(i, j^{\prime}\right)$, giving $(H 4)$.

Since $\sqrt{\beta} n \leq b 4 k \beta n \leq\left|I_{1,1}\right|$ and $\sigma^{\prime}(x) \neq 0$ for each $x$ in the first $\sqrt{\beta} n$ vertices of $\mathcal{L}$, it follows directly from the definition of $f$ that $f(x)=(1, \sigma(x))$, which shows Property (H5).

Finally, we show that Property $(H 6)$ holds with positive probability. Let $i \in[r]$ and $j \in[k]$. We define the random variable $\mathcal{E}_{i, j}:=\left|\left\{x \in f^{*-1}(i, j): \operatorname{deg}(x) \leq 2 D\right\}\right|$. Since $H$ is $D$-degenerate and $\mathcal{L}$ is a labelling of bandwidth at most $\beta n$ we have

$$
e\left(S_{i}^{*}, V(H)\right) \leq D\left|S_{i}^{*}\right|+D 4 \beta n \leq D(1+1 /(4 D))\left|S_{i}^{*}\right| .
$$

Hence, it must hold that $\left|\left\{x \in S_{i}^{*}: \operatorname{deg}(x) \geq 2 D+1\right\}\right|(2 D+1) \leq 2 D(1+1 /(4 D))\left|S_{i}^{*}\right|$. This yields $\left|\left\{x \in S_{i}^{*}: \operatorname{deg}(x) \leq 2 D\right\}\right| \geq\left|S_{i}^{*}\right| /(6 D)$ and therefore

$$
\mathbb{E}\left[\varepsilon_{i, j}\right] \geq \frac{1}{6 k D}\left|S_{i}^{*}\right| \geq \frac{1}{6 D}\left(s_{i}-2\right)(b-2) 4 \beta n .
$$

By applying Chernoff's Inequality (Theorem 20) and using Equations (5.1) and (5.2) as well as $\alpha=$ $1 /(24 D)$ we get

$$
\begin{aligned}
& \mathbb{P}\left[\left|\left\{x \in f^{-1}(i, j): \operatorname{deg}(x) \leq 2 D\right\}\right|<\alpha\left|f^{-1}(i, j)\right|\right] \stackrel{(H 1)}{\leq} \mathbb{P}\left[\mathcal{E}_{i, j}<\alpha\left(s_{i} b 4 \beta n+2 \xi n\right)\right] \\
& \leq \mathbb{P}\left[\mathcal{E}_{i, j}<2 \alpha\left(\left(s_{i}-2\right)(b-2) 4 \beta n\right)\right] \leq \mathbb{P}\left[\mathcal{E}_{i, j}<\frac{1}{2} \mathbb{E}\left[\mathcal{E}_{i, j}\right]\right]<2 \exp \left\{-\frac{\left(s_{i}-2\right)(b-2) 4 \beta n}{72}\right\} .
\end{aligned}
$$

Taking the union bound over all $i \in[r]$ and $j \in[k]$ yields that Property $(H 6)$ holds with positive probability. 
The BANDWIDTh TheOREM IN SPARSE GRAPHS

\section{The common neighbourhood lemma}

In order to prove Lemma 28 we need the following version of the Sparse Regularity Lemma, allowing for a partition equitably refining an initial partition with parts of very different sizes. Given a partition $V(G)=V_{1} \cup \cdots \cup V_{s}$, we say a partition $\left\{V_{i, j}\right\}_{i \in[s], j \in[t]}$ is an equitable $(\varepsilon, p)$-regular refinement of $\left\{V_{i}\right\}_{i \in[s]}$ if $\left|V_{i, j}\right|=\left|V_{i, j^{\prime}}\right| \pm 1$ for each $i \in[s]$ and $j, j^{\prime} \in[t]$, and there are at most $\varepsilon s^{2} t^{2}$ pairs $\left(V_{i, j}, V_{i^{\prime}, j^{\prime}}\right)$ which are not $(\varepsilon, 0, p)$-regular.

Lemma 31. For each $\varepsilon>0$ and $s \in \mathbb{N}$ there exists $t_{1} \geq 1$ such that the following holds. Given any graph $G$, suppose $V_{1} \cup \cdots \cup V_{s}$ is a partition of $V(G)$. Suppose that $e\left(V_{i}\right) \leq 3 p\left|V_{i}\right|^{2}$ for each $i \in[s]$, and $e\left(V_{i}, V_{i^{\prime}}\right) \leq 2 p\left|V_{i}\right|\left|V_{i^{\prime}}\right|$ for each $i \neq i^{\prime} \in[s]$. Then there exist sets $V_{i, 0} \subseteq V_{i}$ for each $i \in[s]$ with $\left|V_{i, 0}\right|<\varepsilon\left|V_{i}\right|$, and an equitable $(\varepsilon, p)$-regular refinement $\left\{V_{i, j}\right\}_{i \in[s], j \in[t]}$ of $\left\{V_{i} \backslash V_{i, 0}\right\}_{i \in[s]}$ for some $t \leq t_{1}$.

The proof is standard, following Scott's method [40]. We defer it to Appendix A.

To prove Lemma 28, we work as follows. First, we choose a regularity parameter $\varepsilon_{0}^{* *}$ and apply Lemma 31 with $\varepsilon_{0}^{* *}$ and the initial partition $V_{1} \backslash W, \ldots, V_{k} \backslash W, W$. From this partition, all we need is a part $W^{\prime} \subseteq W$ and parts $V_{i}^{\prime} \subseteq V_{i} \backslash W$ for each $i \in[k]$, such that each pair $\left(W^{\prime}, V_{i}^{\prime}\right)$ is $\left(\varepsilon_{0}^{* *}, d / 2, p\right)$-lower-regular, which we find by averaging. We now choose our vertices $w_{1}, \ldots, w_{\Delta}$ sequentially (in Claim 32), such that the desired (W1)-(W4) hold for all subsets of the so far chosen vertices at each stage. This is in spirit very much like the usual dense case 'Key Lemma' sequential embedding of vertices using regularity, but in the sparse setting here we need to work somewhat harder and use the regularity inheritance lemmas to show that we can choose vertices which give us lower-regular pairs for future embedding (rather than this being automatic from the slicing lemma, as it is in the dense case).

Thus, the proof mainly amounts to showing that the number of vertices which break one of the desired properties and which we therefore cannot choose is always much smaller than $\left|W^{\prime}\right|$. In order to show this for $(W 1)$ we need to maintain some extra properties, specifically sizes of $G$ - and $\Gamma$-neighbourhoods of chosen vertices within each $V_{i}^{\prime}$, and that these $\Gamma$-neighbourhoods of chosen vertices in each $V_{i}^{\prime}$ form lower-regular pairs with $W^{\prime}$.

Note that the way we choose our various regularity parameters amounts to ensuring that, even after $\Delta-1$ successive applications of regularity inheritance lemmas, we still have sufficient regularity for our argument. Furthermore, it is important to note that the choice of $\varepsilon_{0}^{* *}$ does not have anything to to with $\varepsilon^{*}$ or $\varepsilon_{0}$, rather it affects only the returned value of $\alpha$.

Proof of Lemma 28. First we fix all constants that we need throughout the proof. Given $d>0, k \geq 1$, and $\Delta \geq 2$, let $\varepsilon_{\Delta}^{* *}:=8^{-\Delta} \frac{1}{(k+1)^{2}}\left(\frac{d}{8}\right)^{\Delta}$. Now, for each $j=1, \ldots, \Delta$ sequentially, choose $\varepsilon_{\Delta-j}^{* *} \leq \varepsilon_{\Delta-j+1}^{* *}$ not larger than the $\varepsilon_{0}$ returned by Lemma 17 for input $\varepsilon_{\Delta-j}^{* *}$ and $\frac{d}{2}$.

Now, Lemma 31 with input $\varepsilon_{0}^{* *}$ and $s=k+1$ returns $t_{1} \geq 1$. We set

$$
\alpha:=\frac{1}{2 t_{1}}\left(\frac{d}{4}\right)^{\Delta} .
$$

Next, given $\varepsilon^{*}>0$, let $\varepsilon_{\Delta-1, \Delta-1}^{*}:=\varepsilon^{*}$, and let $\varepsilon_{j, \Delta}^{*}=\varepsilon_{\Delta, j}^{*}=1$ for each $1 \leq j \leq \Delta$. For each $\left(j, j^{\prime}\right) \in$ $[\Delta]^{2} \backslash\{(1,1)\}$ in lexicographic order sequentially, we choose

$$
\varepsilon_{\Delta-j, \Delta-j^{\prime}}^{*} \leq \min \left\{\varepsilon_{\Delta-j+1, \Delta-j^{\prime}}^{*}, \varepsilon_{\Delta-j, \Delta-j^{\prime}+1}^{*}, \varepsilon_{\Delta-j+1, \Delta-j^{\prime}+1}^{*}\right\}
$$


not larger than the $\varepsilon_{0}$ returned by Lemma 17 for both input $\varepsilon_{\Delta-j+1, \Delta-j^{\prime}}^{*}$ and $d$, and for input $\varepsilon_{\Delta-j, \Delta-j^{\prime}+1}^{*}$ and $d$, and not larger than the $\varepsilon_{0}$ returned by Lemma 18 for input $\varepsilon_{\Delta-j+1, \Delta-j^{\prime}+1}^{*}$ and $d$.

We choose $\varepsilon_{0}$ small enough such that $\left(1+\varepsilon_{0}\right)^{\Delta} \leq 1+\varepsilon^{*}$ and $\left(1-\varepsilon_{0}\right)^{\Delta} \geq 1-\varepsilon^{*}$. Given $r \geq 1$ and $\varepsilon$ with $0<\varepsilon \leq \varepsilon_{0}$, suppose that $C$ is large enough for each of these calls to Lemmas 17 and 18, and for Proposition 19 with input $\varepsilon_{0}$. Finally, we set

$$
C^{*}=10^{12} k^{4} t_{1} r^{4} \varepsilon^{-4} 2^{2 \Delta} C .
$$

Given $p \geq C^{*}\left(\frac{\log n}{n}\right)^{1 / \Delta}$, a.a.s. the good events of each of the above calls to Lemma 17 and 18 , and to Proposition 19 and Lemma 31, occur. We condition from now on upon these events occurring for $\Gamma=G(n, p)$.

Let $G=(V, E)$ be a subgraph of $\Gamma$. Suppose $\left\{V_{i}\right\}_{i \in[k]}$ and $W$ satisfy the conditions of the lemma. We first apply Lemma 31, with the promised input parameters $\varepsilon_{0}^{* *}$ and $s=k+1$, to $G\left[V_{1} \cup \cdots \cup V_{k} \cup W\right]$, with input partition $\left\{V_{i} \backslash W\right\}_{i \in[k]} \cup\{W\}$. We can do this because $C p^{-1} \log n<10^{-10} \frac{\varepsilon^{4} p n}{k^{4} r^{4}}$, so that the good event of Proposition 19 guarantees that the conditions of Lemma 31 are satisfied. This returns a partition refining each set of $\left\{V_{i} \backslash W\right\}_{i \in[k]} \cup\{W\}$ into $1 \leq t \leq t_{1}$ clusters together with a small exceptional set. Let $W^{\prime} \subseteq W$ be a cluster which is in at most $2 k \varepsilon_{0}^{* *} t$ pairs with clusters in $\left(V_{1} \cup \cdots \cup V_{k}\right) \backslash W$ which are not $\left(\varepsilon_{0}^{* *}, p\right)_{G}$-lower-regular. Such a cluster exists by averaging. By Proposition 19 and $(V 1)$, at most $4(k+1) \varepsilon_{0}^{* *} p \frac{4 n}{r}\left|W^{\prime}\right|$ edges lie in the pairs between $W^{\prime}$ and the $V_{i}$ which are not lower-regular, and by Proposition 19 and (V3) at most $2 p|W|\left|W^{\prime}\right|<\varepsilon_{0}^{* *} p \frac{n}{r}\left|W^{\prime}\right|$ edges leaving $W^{\prime}$ lie in $W$. By $(V 4)$, for each $i \in[k]$ each $w \in W^{\prime}$ has at least $d p\left|V_{i}\right|$ neighbours in $V_{i}$, and hence there are at least $\frac{d p}{2}\left|V_{i}\right|\left|W^{\prime}\right|$ edges from $W^{\prime}$ to $V_{i} \backslash W$ which lie in $\left(\varepsilon_{0}^{* *}, p\right)_{G^{-}}$-lower-regular pairs. By averaging, for each $i \in[k]$ there exists a cluster $V_{i}^{\prime}$ of the partition such that $\left(W^{\prime}, V_{i}^{\prime}\right)$ is $\left(\varepsilon_{0}^{* *}, d / 2, p\right)_{G}$-lower-regular. For the remainder of the proof, we will only need these $k+1$ clusters from the partition.

Notice that for every $i \in[k]$ we have

$$
\left|V_{i}\right| \geq\left|V_{i}^{\prime}\right| \geq \frac{n}{8 k t_{1} r} \geq \frac{1}{8 k t_{1} r}\left(C^{*}\right)^{2} p^{-2} \log n \geq C^{*} p^{-2} \log n
$$

and

$$
\left|W^{\prime}\right| \geq 10^{-11} \frac{\varepsilon^{4} p n}{t_{1} k^{4} r^{4}} \geq 10^{-11} \frac{\varepsilon^{4}}{t_{1} k^{4} r^{4}}\left(C^{*}\right)^{2} p^{-1} \log n \geq C^{*} p^{-1} \log n
$$

both by the choice of $C^{*}$ and $p$.

We choose the $\Delta$-tuple $\left(w_{1}, \ldots, w_{\Delta}\right)$ inductively, using the following claim.

Claim 32. For each $0 \leq \ell \leq \Delta$ there exists an $\ell$-tuple $\left(w_{1}, \ldots, w_{\ell}\right) \in\left(\begin{array}{c}W^{\prime} \\ \ell\end{array}\right)$ such that the following holds. For every $\Lambda, \Lambda^{*} \subseteq[\ell]$, and every $i \neq i^{\prime} \in[k]$ we have

(L1) $\left(\bigcap_{j \in \Lambda} N_{\Gamma}\left(w_{j}, V_{i}^{\prime}\right), W^{\prime}\right)$ is $\left(\varepsilon_{|\Lambda|}^{* *}, \frac{d}{2}, p\right)_{G}$-lower-regular if $|\Lambda|<\Delta$,

(L2) $\left|\bigcap_{j \in \Lambda} N_{G}\left(w_{j}, V_{i}^{\prime}\right)\right| \geq\left(\frac{d}{4}\right)^{|\Lambda|} p^{|\Lambda|}\left|V_{i}^{\prime}\right|$,

(L3) $\left|\bigcap_{j \in \Lambda} N_{\Gamma}\left(w_{j}\right)\right| \leq\left(1+\varepsilon_{0}\right)^{|\Lambda|} p^{|\Lambda|} n$,

(L4) $\left|\bigcap_{j \in \Lambda} N_{\Gamma}\left(w_{j}, V_{i}^{\prime}\right)\right|=\left(1 \pm \varepsilon_{0}\right)^{|\Lambda|} p^{|\Lambda|}\left|V_{i}^{\prime}\right|$, 
(L5) $\left|\bigcap_{j \in \Lambda} N_{\Gamma}\left(w_{j}, V_{i}\right)\right|=\left(1 \pm \varepsilon_{0}\right)^{|\Lambda|} p^{|\Lambda|}\left|V_{i}\right|$, and

(L6) $\left(\bigcap_{j \in \Lambda} N_{\Gamma}\left(w_{j}, V_{i}\right), \bigcap_{j^{*} \in \Lambda^{*}} N_{\Gamma}\left(w_{j^{*}}, V_{i^{\prime}}\right)\right)$ is $\left(\varepsilon_{|\Lambda|, \mid \Lambda^{*}}^{*}, d, p\right)_{G^{-}}$-lower-regular if $|\Lambda|,\left|\Lambda^{*}\right|<\Delta$ and either $\Delta \geq 3$ or $\Lambda \cap \Lambda^{*}=\varnothing$ or both.

We prove this claim by induction on $\ell$. Recall that if $\Lambda=\emptyset$ then $\bigcap_{j \in \Lambda} N_{\Gamma}\left(w_{j}, V_{i}^{\prime}\right)$ is by definition equal to $V_{i}^{\prime}$, and that $[0]=\emptyset$.

Proof of Claim 32. For the base case $\ell=0$, observe that $(L 1)$ follows from our choice of $W^{\prime}$ and the $V_{i}^{\prime}$. For every $i, j \in[k]$, the pair $\left(V_{i}, V_{j}\right)$ is $(\varepsilon, d, p)_{G}$-lower-regular by $(V 2)$, and since $\varepsilon \leq \varepsilon_{0,0}^{*}$ this gives $(L 6)$. The remaining three properties (L2), (L4) and (L5) are tautologies for $\ell=0$.

For the inductive step, suppose that for some $0 \leq \ell<\Delta$ there exists an $\ell$-tuple $\left(w_{1}, \ldots, w_{\ell}\right) \in\left(\begin{array}{c}W^{\prime} \\ \ell\end{array}\right)$ satisfying $(L 1)-(L 6)$. We now find a vertex $w_{\ell+1} \in W^{\prime}$ such that the $(\ell+1)$-tuple $\left(w_{1}, \ldots, w_{\ell+1}\right)$ still satisfies $(L 1)-(L 6)$. We do this by determining, for each of these five conditions, an upper bound on the number of vertices in $W^{\prime}$ that violate them and show that the sum of these upper bounds is less than $\left|W^{\prime}\right|-\ell$.

Suppose $\Lambda \subseteq[\ell]$ satisfies $|\Lambda|<\Delta-1$, and suppose $i \in[k]$. By the choice of $C$ and $p$ we have for every $i \in[k]$

$$
\left|\bigcap_{j \in \Lambda} N_{\Gamma}\left(w_{j}, V_{i}^{\prime}\right)\right| \stackrel{(L 4)}{\geq}\left(1-\varepsilon_{0}\right)^{|\Lambda|} p^{|\Lambda|}\left|V_{i}^{\prime}\right| \stackrel{|\Lambda|<\Delta-1}{\geq}\left(1-\varepsilon_{0}\right)^{\Delta-2} p^{\Delta-2} \frac{n}{8 k t r} \geq C p^{-2} \log n .
$$

We also have $\left|W^{\prime}\right| \geq C^{*} p^{-1} \log n$ by (6.1) and $\left(\bigcap_{j \in \Lambda} N_{\Gamma}\left(w_{j}, V_{i}^{\prime}\right), W^{\prime}\right)$ is an $\left(\varepsilon_{|\Lambda|}^{* *}, d / 2, p\right)_{G^{-}}$-lower-regular pair by $(L 1)$. Since the good event of Lemma 17 with input $\varepsilon_{|\Lambda|+1}^{* *}$ and $\frac{d}{2}$ occurs, there exist at most $C p^{-1} \log n$ vertices $w$ in $W^{\prime}$ such that

$$
\left(\bigcap_{j \in \Lambda} N_{\Gamma}\left(w_{j}, V_{i}^{\prime}\right) \cap N_{\Gamma}(w), W^{\prime}\right)=\left(\bigcap_{j \in \Lambda} N_{\Gamma}\left(w_{j}, V_{i}^{\prime}\right) \cap N_{\Gamma}\left(w, V_{i}^{\prime}\right), W^{\prime}\right)
$$

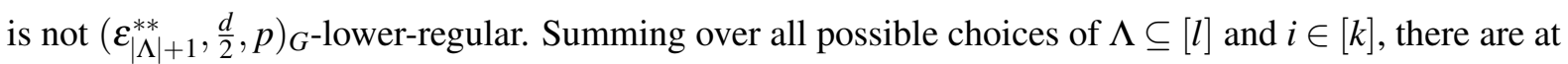
most $2^{\Delta} k^{2} C p^{-1} \log n$ vertices $w$ in $W^{\prime}$ such that $\left(w_{1}, \ldots, w_{l}, w\right)$ does not satisfy $(L 1)$.

Moving on to (L2), let $\Lambda \subseteq[\ell]$ and $i \in[k]$ be given. We have

$$
\begin{aligned}
& \left|\bigcap_{j \in \Lambda} N_{G}\left(w_{j}, V_{i}^{\prime}\right)\right| \stackrel{(L 2)}{\geq}\left(\frac{d}{4}\right)^{|\Lambda|} p^{|\Lambda|}\left|V_{i}^{\prime}\right| \quad \text { and } \\
& \left|\bigcap_{j \in \Lambda} N_{\Gamma}\left(w_{j}, V_{i}^{\prime}\right)\right| \stackrel{(L 4)}{\leq}\left(1+\varepsilon_{0}\right)^{|\Lambda|} p^{|\Lambda|}\left|V_{i}^{\prime}\right| .
\end{aligned}
$$

By choice of $\varepsilon_{0}$ and $\varepsilon_{|\Lambda|}^{* *}$, we thus have $\left|\bigcap_{j \in \Lambda} N_{G}\left(w_{j}, V_{i}^{\prime}\right)\right| \geq \varepsilon_{|\Lambda|}^{* *}\left|\bigcap_{j \in \Lambda} N_{\Gamma}\left(w_{j}, V_{i}^{\prime}\right)\right|$. Now by $(L 1)$, the pair $\left(W^{\prime}, \bigcap_{j \in \Lambda} N_{\Gamma}\left(w_{j}, V_{i}^{\prime}\right)\right)$ is $\left(\varepsilon_{|\Lambda|}^{* *}, \frac{d}{2}, p\right)_{G}$-lower-regular, and thus the number of vertices $w \in W^{\prime}$ such that

$$
\left|N_{G}\left(w, V_{i}^{\prime}\right) \cap \bigcap_{j \in \Lambda} N_{G}\left(w_{j}, V_{i}^{\prime}\right)\right|<\left(\frac{d}{4}\right)^{|\Lambda|+1} p^{|\Lambda|+1}\left|V_{i}^{\prime}\right|
$$


is at most $\varepsilon_{|\Lambda|}^{* *}\left|W^{\prime}\right| \leq \varepsilon_{\Delta}^{* *}\left|W^{\prime}\right|$. Summing over the choices of $\Lambda \subseteq[\ell]$ and $i \in[k]$, the number of $w \in W^{\prime}$ violating (L2) is at most $2^{\Delta} k \varepsilon_{\Delta}^{* *}\left|W^{\prime}\right|$.

For $(L 4)$, given $\Lambda \subseteq[\ell]$ and $i \in[k]$, by $(L 4)$ we have

$$
\left|\bigcap_{j \in \Lambda} N_{\Gamma}\left(w_{j}, V_{i}^{\prime}\right)\right|=\left(1 \pm \varepsilon_{0}\right)^{|\Lambda|} p^{|\Lambda|}\left|V_{i}^{\prime}\right|
$$

and by choice of $\varepsilon_{0}$ and $p$, in particular $\left|\bigcap_{j \in \Lambda} N_{\Gamma}\left(w_{j}, V_{i}^{\prime}\right)\right| \geq C p^{-1} \log n$. Since the good event of Proposition 19 occurs, the number of vertices $w \in W^{\prime}$ such that $\left|N_{\Gamma}\left(w, V_{i}^{\prime}\right) \cap \bigcap_{j \in \Lambda} N_{\Gamma}\left(w_{j}, V_{i}^{\prime}\right)\right|$ is either smaller than $\left(1-\varepsilon_{0}\right)^{|\Lambda|+1} p^{|\Lambda|+1}\left|V_{i}^{\prime}\right|$ or larger than $\left(1+\varepsilon_{0}\right)^{|\Lambda|+1} p^{|\Lambda|+1}\left|V_{i}^{\prime}\right|$ is at most $2 C p^{-1} \log n$. Summing over the choices of $\Lambda \subseteq[\ell]$ and of $i \in[k]$, we conclude that at most $2^{\Delta+1} k C p^{-1} \log n$ vertices of $W^{\prime}$ violate $(L 4)$. Since $n \geq\left|V_{i}\right| \geq\left|V_{i}^{\prime}\right|$, the same calculation shows that a further at most $2^{\Delta+1} k C p^{-1} \log n$ vertices of $W^{\prime}$ violate $(L 5)$, and at most $2^{\Delta+1} k C p^{-1} \log n$ vertices of $W^{\prime}$ violate (L3).

Finally, we come to (L6). Suppose we are given $\Lambda, \Lambda^{\prime} \subseteq[\ell]$ and distinct $i, i^{\prime} \in[k]$. Suppose that $|\Lambda| \leq \Delta-2$ and $\left|\Lambda^{\prime}\right| \leq \Delta-1$. We wish to show that for most vertices $w \in W^{\prime}$, the pair $\left(N_{\Gamma}\left(w, V_{i}\right) \cap\right.$ $\left.\bigcap_{j \in \Lambda} N_{\Gamma}\left(w_{j}, V_{i}\right), \bigcap_{j \in \Lambda} N_{\Gamma}\left(w_{j}, V_{i}^{\prime}\right)\right)$ is $\left(\varepsilon_{|\Lambda|+1,\left|\Lambda^{\prime}\right|}^{*}, d, p\right)_{G}$-lower-regular, and furthermore, if $\Delta \geq 3$ and $\left|\Lambda^{\prime}\right| \leq \Delta-2$, that the pair

$$
\left(N_{\Gamma}\left(w, V_{i}\right) \cap \bigcap_{j \in \Lambda} N_{\Gamma}\left(w_{j}, V_{i}\right), N_{\Gamma}\left(w, V_{i^{\prime}}\right) \cap \bigcap_{j \in \Lambda} N_{\Gamma}\left(w_{j}, V_{i}^{\prime}\right)\right)
$$

is $\left(\varepsilon_{|\Lambda|+1,\left|\Lambda^{\prime}\right|+1}^{*}, d, p\right)_{G}$-lower-regular.

By $(L 5)$, and by choice of $\varepsilon_{0}, C$ and $p$, we have

$$
\begin{aligned}
& \left|\bigcap_{j \in \Lambda} N_{\Gamma}\left(w_{j}, V_{i}\right)\right| \geq\left(1-\varepsilon_{0}\right)^{|\Lambda|} p^{|\Lambda|}\left|V_{i}\right| \geq C p^{|\Lambda|-\Delta} \log n \quad \text { and } \\
& \left|\bigcap_{j \in \Lambda^{\prime}} N_{\Gamma}\left(w_{j}, V_{i^{\prime}}\right)\right| \geq\left(1-\varepsilon_{0}\right)^{\left|\Lambda^{\prime}\right|} p^{\left|\Lambda^{\prime}\right|}\left|V_{i^{\prime}}\right| \geq C p^{\left|\Lambda^{\prime}\right|-\Delta} \log n .
\end{aligned}
$$

By (L6), the pair $\left(\bigcap_{j \in \Lambda} N_{\Gamma}\left(w_{j}, V_{i}\right), \bigcap_{j \in \Lambda} N_{\Gamma}\left(w_{j}, V_{i}^{\prime}\right)\right)$ is $\left(\varepsilon_{|\Lambda|,\left|\Lambda^{\prime}\right|}^{*}, d, p\right)_{G}$-lower-regular. Since the good event of Lemma 17 with input $\varepsilon_{|\Lambda|+1,\left|\Lambda^{\prime}\right|}^{*}$ and $d$ occurs, there are at most $C p^{-1} \log n$ vertices $w$ of $W^{\prime}$ such that $\left(N_{\Gamma}\left(w, V_{i}\right) \cap \bigcap_{j \in \Lambda} N_{\Gamma}\left(w_{j}, V_{i}\right), \bigcap_{j \in \Lambda} N_{\Gamma}\left(w_{j}, V_{i}^{\prime}\right)\right)$ is not $\left(\varepsilon_{|\Lambda|+1,\left|\Lambda^{\prime}\right|}^{*}, d, p\right)_{G}$-lower-regular. Furthermore, if $\left|\Lambda^{\prime}\right| \leq \Delta-2$, then since the good event of Lemma 18 with input $\varepsilon_{|\Lambda|+1,\left|\Lambda^{\prime}\right|+1}^{*}$ and $d$ occurs, there are at most $C p^{-2} \log n$ vertices $w$ of $W^{\prime}$ such that

$$
\left(N_{\Gamma}\left(w, V_{i}\right) \cap \bigcap_{j \in \Lambda} N_{\Gamma}\left(w_{j}, V_{i}\right), N_{\Gamma}\left(w, V_{i^{\prime}}\right) \cap \bigcap_{j \in \Lambda} N_{\Gamma}\left(w_{j}, V_{i}^{\prime}\right)\right) \text { is not }\left(\varepsilon_{|\Lambda|+1,\left|\Lambda^{\prime}\right|}^{*}, d, p\right)_{G} \text {-lower-regular. }
$$

Observe that if $\Delta=2$ the property ( $L 6)$ does not require this pair to be lower-regular. Summing over the choices of $\Lambda, \Lambda^{\prime} \subseteq[\ell]$ and $i, i^{\prime} \in[k]$, we conclude that if $\Delta=2$ then at most $2^{2 \Delta} k^{2} C p^{-1} \log n$ vertices $w$ of $W^{\prime}$ cause ( $\left.L 6\right)$ to fail, while if $\Delta \geq 3$, at most $2^{2 \Delta} k^{2} C\left(p^{-1}+p^{-2}\right) \log n$ vertices $w$ of $W^{\prime}$ violate $(L 6)$.

Summing up, if $\Delta=2$ then at most

$$
2^{\Delta} k^{2} C p^{-1} \log n+2^{\Delta} k \varepsilon_{\Delta}^{* *}\left|W^{\prime}\right|+3 \cdot 2^{\Delta+1} k C p^{-1} \log n+2^{2 \Delta} k^{2} C p^{-1} \log n
$$


THE BANDWIDTH THEOREM IN SPARSE GRAPHS

vertices $w$ of $W^{\prime}$ cannot be chosen as $w_{\ell+1}$. By choice of $C^{*}$ and $\varepsilon_{\Delta}^{* *}$, and by choice of $p$, this is at most $\frac{1}{2}\left|W^{\prime}\right|$, so that there exists a vertex of $W^{\prime}$ which can be chosen as $w_{\ell+1}$, as desired. If on the other hand $\Delta \geq 3$, then at most

$$
2^{\Delta} k^{2} C p^{-1} \log n+2^{\Delta} k \varepsilon_{\Delta}^{* *}\left|W^{\prime}\right|+3 \cdot 2^{\Delta+1} k C p^{-1} \log n+2^{2 \Delta} k^{2} C\left(p^{-1}+p^{-2}\right) \log n
$$

vertices of $W^{\prime}$ cannot be chosen as $w_{\ell+1}$. Again by choice of $C^{*}, \varepsilon_{\Delta}^{* *}$ and $p$, this is at most $\frac{1}{2}\left|W^{\prime}\right|$, and again we therefore can choose $w_{\ell+1}$ satisfying $(L 1)-(L 6)$ as desired.

Finally, let us argue why the lemma is a consequence of Claim 32. Let $\left(w_{1}, \ldots, w_{\Delta}\right) \in\left(\begin{array}{c}W^{\prime} \\ \Delta\end{array}\right)$ be a tuple satisfying (L1)-(L6). By (L2), for any $\Lambda \subseteq[\ell]$ and $i \in[k]$ we have

$$
\left|\bigcap_{j \in \Lambda} N_{G}\left(w_{j}, V_{i}\right)\right| \geq\left(\frac{d}{4}\right)^{|\Lambda|} p^{|\Lambda|}\left|V_{i}^{\prime}\right| \geq\left(\frac{d}{4}\right)^{\Delta} p^{|\Lambda|} \frac{\left|V_{i}\right|}{2 t_{1}} \geq \alpha p^{|\Lambda|}\left|V_{i}\right|,
$$

as required for (W1). Properties (W2), (W3) and (W4) are respectively (L3), (L5) and (L6), by choice of $\varepsilon_{0}$.

\section{The balancing lemma}

The statement of Lemma 29 gives us a partition of $V(G)$ with parts $\left(V_{i, j}\right)_{i \in[r], j \in[k]}$, and a collection of 'target integers' $\left(n_{i, j}\right)_{i \in[r], j \in[k]}$, with each $n_{i, j}$ close to $\left|V_{i, j}\right|$, and with $\sum n_{i, j}=\sum\left|V_{i, j}\right|$. Our aim is to find a partition of $V(G)$ with parts $\left(V_{i, j}^{\prime}\right)_{i \in[r], j \in[k]}$ such that $\left|V_{i, j}^{\prime}\right|=n_{i, j}$ for each $i, j$. This partition is required to maintain similar regularity properties as the original partition, while not substantially changing common neighbourhoods of vertices.

There are two steps to our proof. In a first step, we correct global imbalance, that is, we find a partition $\widetilde{\mathcal{V}}$ which maintains all the desired properties and which has the property that $\sum_{i}\left|\widetilde{V}_{i, j}\right|=\sum_{i} n_{i, j}$ for each $j \in[k]$. To do this, we identify some $j^{*}$ such that $\sum_{i}\left|V_{i, j^{*}}\right|>\sum_{i} n_{i, j^{*}}$ and $j^{\prime}$ such that $\sum_{i}\left|V_{i, j^{\prime}}\right|<\sum_{i} n_{i, j^{\prime}}$. We move $\sum_{i}\left|V_{i, j^{*}}\right|-n_{i, j^{*}}$ vertices from $V_{1, j^{*}}$ to some cluster $V_{i^{\prime}, j^{\prime}}$, maintaining the desired properties, and repeat this procedure until no global imbalance remains.

In a second step, we correct local imbalance, that is, for each $i=1, \ldots, r-1$ sequentially, and for each $j \in[k]$, we move vertices between $\widetilde{V}_{i, j}$ and $\widetilde{V}_{i+1, j}$, maintaining the desired properties, to obtain the partition $\mathcal{V}^{\prime}$ such that $\left|V_{i, j}^{\prime}\right|=n_{i, j}$ for each $i, j$. Observe that because $\widetilde{\mathcal{V}}$ is globally balanced, once we know $\left|V_{i, j}^{\prime}\right|=n_{i, j}$ for each $i \in[r-1]$ and each $j \in[k]$ we are guaranteed that $\left|V_{r, j}^{\prime}\right|=n_{r, j}$ for each $j \in[k]$.

The proof of the lemma then comes down to showing that we can move vertices and maintain the desired properties. Because we start with a partition in which $V_{i, j}$ is very close to $n_{i, j}$ for each $i$ and $j$, the total number of vertices we move in any step is at most the sum of the differences, which is much smaller than any $n_{i, j}$. The following lemma shows that we can move any small (compared to all $n_{i, j}$ ) number of vertices from one part to another and maintain the desired properties.

Lemma 33. For all integers $k, r_{1}, \Delta \geq 1$, and reals $d>0$ and $0<\varepsilon<1 / 2 k$ as well as $0<\xi<1 /\left(100 k r_{1}^{3}\right)$, there exists $C^{*}>0$ such that the following holds for all sufficiently large $n$. 
Let $\Gamma$ be a graph on vertex set $[n]$, and let $G$ be a not necessarily spanning subgraph. Let $X, Z_{1}, \ldots, Z_{k-1} \subseteq V(G)$ be pairwise disjoint subsets, each of size at least $n /\left(16 k r_{1}\right)$, such that $\left(X, Z_{i}\right)$ is

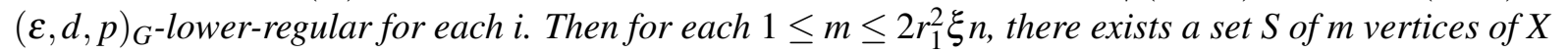
with the following properties.

(SM 1) For each $v \in S$ we have $\operatorname{deg}_{G}\left(v ; Z_{i}\right) \geq(d-\varepsilon) p\left|Z_{i}\right|$ for each $i \in[k-1]$, and

(SM2) for each $1 \leq s \leq \Delta$ and every collection of vertices $v_{1}, \ldots, v_{s} \in[n]$ we have

$$
\operatorname{deg}_{\Gamma}\left(v_{1}, \ldots, v_{s} ; S\right) \leq 100 k r_{1}^{3} \xi \operatorname{deg}_{\Gamma}\left(v_{1}, \ldots, v_{s} ; X\right)+\frac{1}{100} C^{*} \log n .
$$

Proof. Given $k, r_{1}, \Delta, d, \xi$ and $\varepsilon$, let $C$ be returned by Lemma 22 for input $\xi$ and $\Delta$. We set $C^{*}=100 C$. Given $\Gamma, G$ and $X, Y, Z_{1}, \ldots, Z_{k-1}$, let $X^{\prime}$ be the set of vertices $v \in X$ such that $\operatorname{deg}_{G}\left(v ; Z_{i}\right) \geq(d-\varepsilon) p\left|Z_{i}\right|$ for each $i \in[k-1]$. Because each pair $\left(X, Z_{i}\right)$ for $i \in[k-1]$ is $(\varepsilon, d, p)_{G}$-lower-regular, we have $\left|X^{\prime}\right| \geq$ $|X|-k \varepsilon|X| \geq|X| / 2$.

We now apply Lemma 22, with input $\xi, \Delta, W=X^{\prime}$ and the sets $T_{i}$ being the sets $N_{\Gamma}\left(v_{1}, \ldots, v_{s} ; X^{\prime}\right)$ for each $1 \leq s \leq \Delta$ and $v_{1}, \ldots, v_{s} \in[n]$, to choose a set $S$ of size $m \leq 2 r_{1}^{2} \xi_{n} \leq\left|X^{\prime}\right|$ in $X^{\prime}$. We then have

$$
\begin{aligned}
\operatorname{deg}_{\Gamma}\left(v_{1}, \ldots, v_{s} ; S\right) & \leq\left(\frac{2 r_{1}^{2} \xi n}{\left|X^{\prime}\right|}+\xi\right) \operatorname{deg}_{\Gamma}\left(v_{1}, \ldots, v_{s} ; X^{\prime}\right)+C \log n \\
& \leq 100 k r_{1}^{3} \xi \operatorname{deg}_{\Gamma}\left(v_{1}, \ldots, v_{s} ; X\right)+\frac{1}{100} C^{*} \log n
\end{aligned}
$$

where the final inequality is by choice of $C^{*}$, and since $\left|X^{\prime}\right| \geq|X| / 2 \geq n /\left(32 k r_{1}\right)$. Thus the set $S$ satisfies ( $S M 2)$, and since $S \subseteq X^{\prime}$ we have $(S M 1)$.

We now prove the balancing lemma.

Proof of Lemma 29. Given integers $k, r_{1}, \Delta \geq 1$ and reals $\gamma, d>0$ and $0<\varepsilon<\min \{d, 1 /(2 k)\}$, we set

$$
\xi=10^{-15} \varepsilon^{4} d /\left(k^{3} r_{1}^{5}\right)
$$

Let $C_{1}^{*}$ be returned by Lemma 33 with input $k, r_{1}, \Delta, d, \varepsilon / 4$ and $\xi$, and let $C_{2}^{*}$ be returned by Lemma 33 with input $k, r_{1}, \Delta, d, 3 \varepsilon / 4$ and $\xi$. We set $C^{*}=\max \left\{C_{1}^{*}, C_{2}^{*}\right\}$.

Now suppose that $p \geq C^{*}\left(\frac{\log n}{n}\right)^{1 / 2}$, that $10 \gamma^{-1} \leq r \leq r_{1}$, and that graphs $\Gamma$ and $G$, a partition $\mathcal{V}$ of $V=V(G)$, and graphs $R_{r}^{k}, B_{r}^{k}$ and $K_{r}^{k}$ on $[r] \times[k]$ as in the statement of Lemma 29 are given.

First stage (global imbalance):

We use the following algorithm.

In each step where we select $S$, we make use of Lemma 33 to do so, with input $k, r_{1}, \Delta, d$, and $\varepsilon / 4$, with $X=V_{1, j^{*}}$ and with the $Z_{1}, \ldots, Z_{k-1}$ being the $V_{i^{\prime}, j^{\prime \prime}}$ with $j^{\prime \prime} \neq j^{\prime}$.

We claim that the algorithm completes successfully, in other words that each of the choices is possible, and that Lemma 33 is always applicable. In each While loop, since $\sum_{i, j}\left|V_{i, j}\right|-n_{i, j}=0$ and since the While condition is satisfied, $j^{*}$ satisfies $\sum_{i \in[r]}\left|V_{i, j^{*}}\right|-n_{i, j^{*}}>0$.

Observe that the While loop is run at most $k$ times, since at the end of the While loop in which we selected some $j=j^{*}$ we have $\sum_{i \in[r]}\left|V_{i, j^{*}}\right|-n_{i, j^{*}}=0$ and therefore we do not select $j$ as either $j^{*}$ or $j^{\prime}$ 


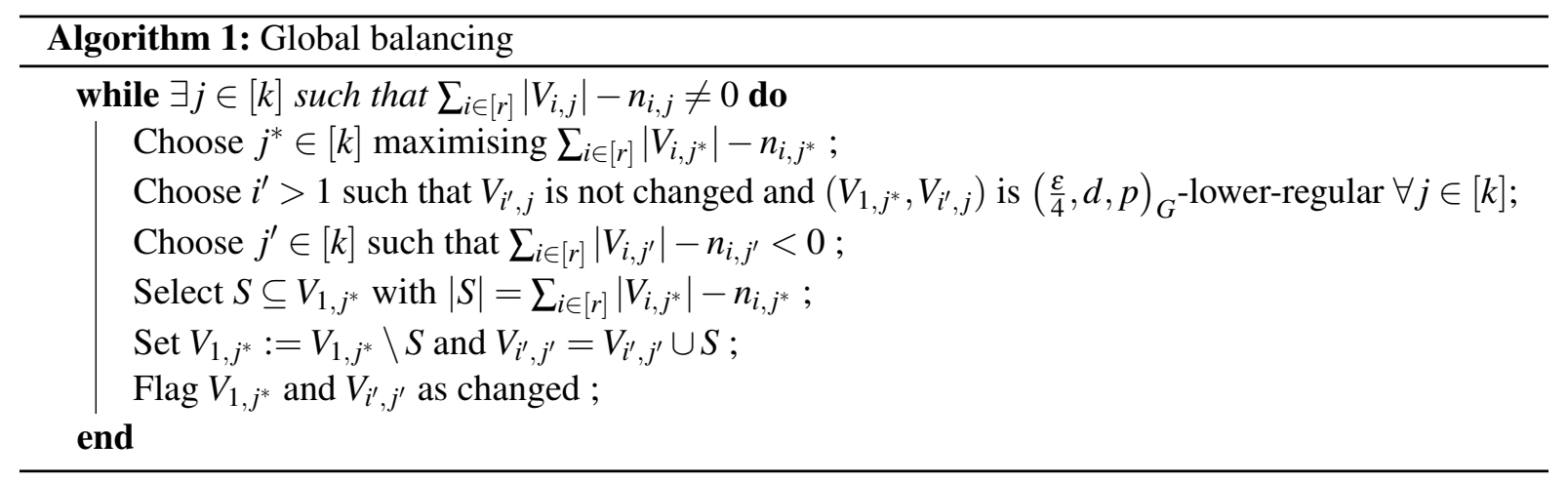

in future iterations. It follows that the number of $V_{i, j}$ flagged as changed never exceeds $2 k$. Now the set $V_{1, j^{*}}$ has degree at least $\left(k-1+\frac{\gamma k}{2}\right) r$ in $R_{r}^{k}$, and so there are at least $\gamma k r / 2$ indices $i \in[r]$ such that $V_{1, j^{*}}$ is adjacent to each $V_{i, j}$ in $R_{r}^{k}$. Since $\gamma k r / 2>3 k$, in particular we can choose $i^{\prime}$ such that $V_{1, j^{*}}$ is adjacent to each $V_{i^{\prime}, j}$ in $R_{r}^{k}$ and no $V_{i^{\prime}, j}$ is flagged as changed. It follows that each pair $\left(V_{1, j^{*}}, V_{i^{\prime}, j}\right)$ is $\left(\frac{\varepsilon}{4}, d, p\right)_{G}$-lower-regular and thus it is possible to choose $i^{\prime}$. It is possible to choose $j^{\prime}$ since the While condition holds. Finally, we need to show that Lemma 33 is always applicable with the given parameters. In each application, the sets denoted $X, Z_{1}, \ldots, Z_{k-1}$ are parts of the partition $\mathcal{V}$ (so they were not changed by the algorithm yet). It follows that each set has size at least $n /(8 k r)>n /\left(16 k r_{1}\right)$. Since $\mathcal{V}$ is $\left(\frac{\varepsilon}{4}, d, p\right)$ lower-regular on $B_{k}^{r}$, the pairs $\left(X, Z_{1}\right), \ldots,\left(X, Z_{k-1}\right)$ are $\left(\frac{\varepsilon}{4}, d, p\right)$-lower-regular as required. Finally, by choice of $j^{*}$ we see that the sizes of the sets $S$ we select in each step are decreasing, so it is enough to show that in the first step we have $|S| \leq r \xi n$, which follows from (B 1). Thus Lemma 33 is applicable in each step, and we conclude that the algorithm indeed completes. We denote the resulting vertex partition by $\widetilde{\mathcal{V}}=\left\{\widetilde{V}_{i, j}\right\}_{i \in[r], j \in[k]}$.

Claim 34. We have the following properties.

(P1) For each $i \in[r]$ and $j \in[k]$ we have ||$\widetilde{V}_{i, j}\left|-n_{i, j}\right| \leq 2 r \xi n$,

$(P 2) \widetilde{\mathcal{V}}$ is $\left(\frac{\varepsilon}{2}, d, p\right)_{G}$-lower-regular on $R_{r}^{k}$ and $\left(\frac{\varepsilon}{2}, d, p\right)_{G}$-super-regular on $K_{r}^{k}$,

(P3) For each $i \in[r], j \in[k]$ and $1 \leq s \leq \Delta$ and $v_{1}, \ldots, v_{s} \in[n]$ we have

$$
\left|N_{\Gamma}\left(v_{1}, \ldots, v_{s}, \widetilde{V}_{i, j}\right) \triangle N_{\Gamma}\left(v_{1}, \ldots, v_{s}, V_{i, j}\right)\right| \leq 100 k r_{1}^{3} \xi \operatorname{deg}_{\Gamma}\left(v_{1}, \ldots, v_{s} ; V(G)\right)+\frac{1}{100} C^{*} \log n
$$

Proof. Observe that vertices were removed from or added to each $V_{i, j}$ to form $\widetilde{V}_{i, j}$ at most once in the running of Algorithm 1, and the number of vertices added or removed was at most $r \xi n$. Since $\left|V_{i, j}\right|$ satisfies $(B 1)$, we conclude that $(P 1)$ holds. Furthermore, the vertices added to or removed from $V_{i, j}$ satisfy $(S M 2)$ and therefore $(P 3)$ holds.

Since each set $V_{i, j}$ has size at least $n /(8 k r)$, we can apply Proposition 12 with $\mu=v=8 k r^{2} \xi$ to each edge of $R_{r}^{k}$, concluding that $\widetilde{\mathcal{V}}$ is $\left(\frac{\varepsilon}{2}, d, p\right)_{G}$-lower-regular on $R_{r}^{k}$ since $\frac{\varepsilon}{4}+4 \sqrt{8 k r^{2} \xi}<\frac{\varepsilon}{2}$. Now for any 
$i \in[r]$ and $j \in[k]$, consider $v \in \widetilde{V}_{i, j}$. If $v \notin V_{i, j}$, then we applied Lemma 33 to select $v$, and when we did so no $V_{i, j^{\prime}}$ was flagged as changed by Algorithm 1 . Thus by (SM1) we have

$$
\operatorname{deg}_{G}\left(v ; \widetilde{V}_{i, j^{\prime}}\right)=\operatorname{deg}_{G}\left(v ; V_{i, j^{\prime}}\right) \geq\left(d-\frac{\varepsilon}{4}\right) p\left|V_{i, j^{\prime}}\right|=\left(d-\frac{\varepsilon}{4}\right) p\left|\widetilde{V}_{i, j^{\prime}}\right|
$$

for each $j^{\prime} \neq j$, since $V_{i, j}$ is then flagged as changed and thus $V_{i, j^{\prime}}=\widetilde{V}_{i, j^{\prime}}$ for each $j^{\prime} \neq j$. If on the other hand $v \in V_{i, j}$, then by $(B 2)$ we started with $\operatorname{deg}_{G}\left(v ; V_{i, j^{\prime}}\right) \geq\left(d-\frac{\varepsilon}{4}\right) p\left|V_{i, j^{\prime}}\right|$. By $(S M 2)$ and $(B 4)$, we have

$$
\operatorname{deg}_{G}\left(v ; \widetilde{V}_{i, j^{\prime}}\right) \geq\left(d-\frac{\varepsilon}{4}\right) p\left|V_{i, j^{\prime}}\right|-\frac{\varepsilon^{2}}{1000 k r_{1}}\left(1+\frac{\varepsilon}{4}\right) p\left|V_{i, j^{\prime}}\right|-\frac{1}{100} C^{*} \log n \geq\left(d-\frac{\varepsilon}{2}\right) p\left|\widetilde{V}_{i, j^{\prime}}\right|
$$

where the final inequality follows by choice of $n$ sufficiently large and since $\left|\widetilde{V}_{i, j^{\prime}}\right| \leq\left|V_{i, j^{\prime}}\right|+r \xi n \leq$ $\left(1+\frac{\varepsilon d}{100}\right)\left|V_{i, j^{\prime}}\right|$. We conclude that $\widetilde{\mathcal{V}}$ is $\left(\frac{\varepsilon}{2}, d, p\right)$-super-regular on $K_{r}^{k}$, giving $(P 2)$.

\section{Second stage (local imbalance):}

We use Algorithm 2 to correct the local imbalances in $\widetilde{\mathcal{V}}$.

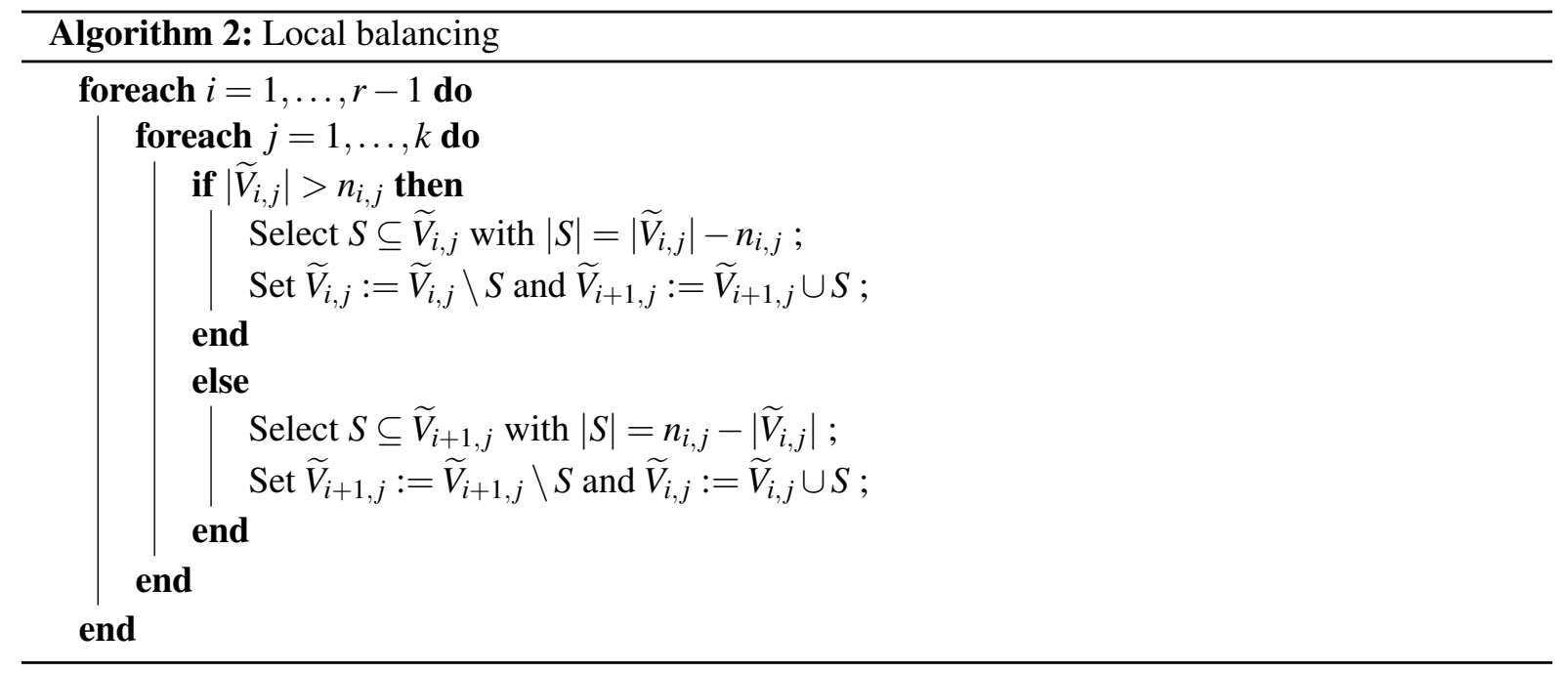

Again, in each step when we select $S$ we make use of Lemma 33 to do so. If we select $S$ from $\widetilde{V}_{i, j}$, then we use input $k, r_{1}, d, 3 \varepsilon / 4$ and $\xi$ with $X=\widetilde{V}_{i, j}$ and the sets $Z_{1}, \ldots, Z_{k-1}$ being $\widetilde{V}_{i+1, j^{\prime}}$ for $j^{\prime} \neq j$. If on the other hand we select $S$ from $\widetilde{V}_{i+1, j}$, then we use input $k, r_{1}, d$ and $3 \varepsilon / 4$, with $X=\widetilde{V}_{i+1, j}$ and the sets $Z_{1}, \ldots, Z_{k-1}$ being $\widetilde{V}_{i, j^{\prime}}$ for $j^{\prime} \neq j$.

We claim that Lemma 33 is always applicable. To see that this is true, observe first that the number of vertices which we move between any $\widetilde{V}_{i, j}$ and $\widetilde{V}_{i+1, j}$ in a given step is by (P1) bounded by $2 k^{2} r^{2} \xi_{n}$. We change any given $\widetilde{V}_{i, j}$ at most twice in the running of the algorithm, so that in total at most $4 k^{2} r^{2} \xi_{n}$ vertices are changed. In particular, we maintain $\left|\widetilde{V}_{i, j}\right| \geq n /\left(16 k r_{1}\right)$ throughout, and, by Proposition 12 , with input $\mu=v=\frac{4 r^{2} \xi n}{n /\left(16 k r_{1}\right)}<100 r_{1}^{3} k \xi$, and using (P2), we maintain the property that any pair in $R_{r}^{k}$, and in particular any pair in $B_{r}^{k}$, is $\left(\frac{3 \varepsilon}{4}, d, p\right)$-lower-regular throughout. This shows that Lemma 33 is 
always applicable, and therefore the algorithm completes and returns a partition $\mathcal{V}^{\prime}$. We claim that this is the desired partition. We need to check that $\left(B 1^{\prime}\right)-\left(B 5^{\prime}\right)$ hold.

Since for each $j \in[k]$ we have $\sum_{i}\left|V_{i, j}^{\prime}\right|=\sum_{i}\left|\widetilde{V}_{i, j}\right|=\sum_{i} n_{i, j}$, and since $\left|V_{i, j}^{\prime}\right|=n_{i, j}$ for each $i \in[r-1]$ and $j \in[k]$, we conclude that $\left|V_{i, j}^{\prime}\right|=n_{i, j}$ for all $i$ and $j$, giving $\left(B 1^{\prime}\right)$.

For the first part of $\left(B 3^{\prime}\right)$, we have justified that we maintain $\left(\frac{3 \varepsilon}{4}, d, p\right)_{G}$-lower-regularity on $R_{r}^{k}$ throughout the algorithm. For the second part, we need to show that for each $i \in[r]$ and $j \neq j^{\prime} \in[k]$, and each $v \in V_{i, j}^{\prime}$, we have $\operatorname{deg}_{G}\left(v ; V_{i, j^{\prime}}^{\prime}\right) \geq(d-\varepsilon) p \mid V_{i, j^{\prime}}^{\prime}$. If $v \in \widetilde{V}_{i, j}$, then by $(P 2)$ we have $\operatorname{deg}_{G}\left(v ; \widetilde{V}_{i, j^{\prime}}\right) \geq$ $\left(d-\frac{\varepsilon}{2}\right) p\left|\widetilde{V}_{i, j^{\prime}}\right|$. We change $\widetilde{V}_{i, j^{\prime}}$ at most twice to obtain $V_{i, j^{\prime}}^{\prime}$, both times by adding or removing vertices satisfying (SM2). As in the proof of Claim (P3) above, using (B4) and (P3) we obtain $\operatorname{deg}_{G}\left(v ; \widetilde{V}_{i, j^{\prime}}\right) \geq$ $(d-\varepsilon) p\left|V_{i, j}^{\prime}\right|$ as desired. If $v \notin \widetilde{V}_{i, j}$, then it was added to the set $\widetilde{V}_{i, j}$ by Algorithm 2 , and $\widetilde{V}_{i, j^{\prime}}$ was changed at most twice thereafter. Again, using (SM 1), (SM2), (B4) and (P3) we obtain $\operatorname{deg}_{G}\left(v ; \widetilde{V}_{i, j^{\prime}}\right) \geq$ $(d-\varepsilon) p\left|V_{i, j}^{\prime}\right|$ as desired.

Now (B2') holds since the total number of vertices moved in Algorithm 1 is at most $k^{2} r \xi n$, in Algorithm 2 at most $4 k^{2} r^{2} \xi n$ vertices are changed in each cluster, and by choice of $\xi$. To see that $\left(B 4^{\prime}\right)$ holds, observe that by (B 4), (P3) and (SM2) we have

$$
\left|N_{\Gamma}\left(v ; V_{i, j}\right) \Delta N_{\Gamma}\left(v ; V_{i, j}^{\prime}\right)\right| \leq \frac{\varepsilon^{2}}{100 k r_{1}} \operatorname{deg}_{\Gamma}(v ; V(G))+\frac{1}{10} C^{*} \log n \leq \frac{\varepsilon^{2}}{50} \operatorname{deg}_{\Gamma}\left(v ; V_{i, j}\right)
$$

where the final inequality follows by choice of $p$ and of $n$ sufficiently large. Using (B3), we can apply Proposition 12, with $\mu=v=\frac{\varepsilon^{2}}{50}$, to deduce $\left(B 4^{\prime}\right)$.

For (B 5'), observe that for any given $i \in[r]$ and $j \in[k]$ we change $\widetilde{V}_{i, j}$ at most twice in the running of Algorithm 2, both times either adding or removing a set satisfying (SM2). By (P3) and choice of $\xi$, we conclude that $\left(B 5^{\prime}\right)$ holds.

Finally, suppose that for any two disjoint vertex sets $A, A^{\prime} \subseteq V(\Gamma)$ with $|A|,\left|A^{\prime}\right| \geq \frac{1}{50000 \mathrm{kr} r_{1}} \varepsilon^{2} \xi$ pn we have $e_{\Gamma}\left(A, A^{\prime}\right) \leq\left(1+\frac{1}{100} \varepsilon^{2} \xi\right) p|A|\left|A^{\prime}\right|$. In each application of Proposition 12 we have $\mu, v \geq \frac{1}{50} \varepsilon^{2} \xi$, and, and if we have 'regular' in place of 'lower-regular' in $(B 2)$, and (B3), we always apply Proposition 12 to a regular pair with sets of size at least $\frac{\varepsilon}{1000 k r_{1}} p n$, so it returns regular pairs for $\left(B 3^{\prime}\right)$, and $\left(B 4^{\prime}\right)$, as desired.

\section{The Bandwidth Theorem in random graphs}

Before embarking on the proof, we first recall from the proof overview (Section 3.1) the main ideas. Given $G$, we first use the lemma for $G$ (Lemma 26) to find a lower-regular partition of $V(G)$, with an extremely small exceptional set $V_{0}$, and whose reduced graph $R_{r}^{k}$ contains a spanning backbone graph $B_{r}^{k}$, on whose subgraph $K_{r}^{k}$ the graph $G$ is super-regular and has one- and two-sided inheritance. Given this, and $H$ together with a $(z, \beta)$-zero-free $(k+1)$-colouring, we use the lemma for $H$ (Lemma 27) to find a homomorphism $f$ from $V(H)$ to $R_{r}^{k}$ almost all of whose edges are mapped to $K_{r}^{k}$ and in which approximately the 'right' number of vertices of $H$ are mapped to each vertex of $R_{r}^{k}$. At this point, if $V_{0}$ were empty, and if the 'approximately' were exact, we would apply the sparse blow-up lemma (Lemma 16) to obtain an embedding of $H$ into $G$. 
Our first aim is to deal with $V_{0}$. We do this one vertex at a time. Given $v \in V_{0}$, we choose $x \in V(H)$ from the first $\beta n$ vertices of the supplied bandwidth order $\mathcal{L}$ which is not in any triangles. We embed $x$ to $v$. We then embed the neighbours of $x$ to carefully chosen neighbours of $v$, which we obtain using Lemma 28. Here we use the fact that $N_{H}(x)$ is independent. This then fixes a clique of $K_{r}^{k}$ to which $N_{H}^{2}(x)$ must be assigned, and gives image restrictions in the corresponding parts of the lower-regular partition for these vertices. Since $N_{H}^{2}(x)$ may have been assigned by $f$ to some quite different clique in $K_{r}^{k}$, we have to adjust $f$ to match. This we can do using the fact, which follows from our assumptions on $\mathcal{L}$, that $x$ is far from vertices of colour zero.

Now the idea is simply to repeat the above procedure, choosing vertices of $V(H)$ to pre-embed which are widely separated in $H$, until we pre-embedded vertices to all of $V_{0}$. We end up with a homomorphism $f^{*}$ from what remains of $V(H)$ to $R_{r}^{k}$. It is easy to check that this homomorphism still maps about the right number of vertices of $H$ to each vertex of $R_{r}^{k}$, simply because $V_{0}$ is small. We now apply the Balancing Lemma (Lemma 29) to correct the sizes of the clusters to match $f^{*}$, and complete the embedding of $H$ using the Sparse Blow-up Lemma (Lemma 16).

There are two difficulties with this idea, the 'subtleties' mentioned in the proof overview (Section 3.1). First, if $\Delta=2$ we might have $\left|V_{0}\right| \gg p n$, so that we should be worried that at some stage of the preembedding we choose $v \in V_{0}$ and discover most or all of its neighbours have already been pre-embedded to. It turns out to be easy to resolve this: we choose each $v \in V_{0}$ not arbitrarily, but by taking those which have least available neighbours first. We will show that this is enough to avoid the problem.

More seriously, because we perform the pre-embedding sequentially, we might use up a significant fraction of $N_{G}(w)$ for some $w \in V(G)$ in the pre-embedding, destroying super-regularity of $G$ on $K_{r}^{k}$, or we might use up a significant fraction of some common neighbourhood which defines an image restriction for the sparse blow-up lemma. In order to avoid this, before we begin the pre-embedding we fix a set $S \subseteq V(G)$ whose size is a very small constant times $n$, chosen using Lemma 22 to not have a large intersection with any $N_{G}(w)$ or with any $\Gamma$-common neighbourhood of at most $\Delta$ vertices of $\Gamma$ (which could define an image restriction). We perform the pre-embedding as outlined above, except that we choose our neighbours of each $v$ within $S$. This procedure is guaranteed not to use up neighbourhood sets guaranteed by super-regularity or image restriction sets, since these sets are all contained in $V \backslash V_{0}$ and even using up all of $S$ would not be enough to do damage.

Proof of Theorem 25. Given $\gamma>0, \Delta \geq 2$ and $k \geq 2$, let $d$ be returned by Lemma 26, with input $\gamma, k$ and $r_{0}:=10 \gamma^{-1}$. Let $\alpha$ be returned by Lemma 28 with input $d, k$ and $\Delta$. We set $D=\Delta$, and let $\varepsilon_{\mathrm{BL}}>0$ and $\rho>0$ be returned by Lemma 16 with input $\Delta, \Delta_{R^{\prime}}=3 k, \Delta_{J}=\Delta, \vartheta=\frac{1}{100 D}, \zeta=\frac{1}{4} \alpha, d$ and $\kappa=64$. Next, putting $\varepsilon^{*}=\frac{1}{8} \varepsilon_{\mathrm{BL}}$ into Lemma 28 returns $\varepsilon_{0}>0$. We choose $\varepsilon=\min \left(\varepsilon_{0}, d, \frac{1}{4 D} \varepsilon^{*}, \frac{1}{100 k}\right)$. Putting $\varepsilon$ into Lemma 26 returns $r_{1}$. Next, Lemma 29, for input $k, r_{1}, \Delta, \gamma, d$ and $8 \varepsilon$, returns $\xi>0$. We assume without loss of generality that $\xi \leq 1 /\left(10 k r_{1}\right)$, and set $\beta=10^{-12} \xi^{2} /\left(\Delta k^{4} r_{1}^{2}\right)$. Let $\mu=\frac{\varepsilon^{2}}{100000 k r}$. Finally, suppose $C^{*}$ is large enough for each of these lemmas, for Lemma 16, for Proposition 19 with input $\varepsilon$, and for Lemma 22 with input $\varepsilon \mu^{2}$ and $\Delta$.

We set $C=10^{10} k^{2} r_{1}^{2} \varepsilon^{-2} \xi^{-1} \Delta^{2 r_{1}+20} \mu^{-\Delta} C^{*}$, and $z=10 / \xi$. Given $p \geq C\left(\frac{\log n}{n}\right)^{1 / \Delta}$, a.a.s. $G(n, p)$ satisfies the good events of Lemma 16, Lemma 26 and Lemma 28, and Proposition 19, with the stated inputs. Suppose that $\Gamma=G(n, p)$ satisfies these good events.

Suppose $G \subseteq \Gamma$ is any spanning subgraph with $\delta(G) \geq\left(\frac{k-1}{k}+\gamma\right) p n$. Let $H$ be a graph on $n$ vertices 
The BANDWIDTh ThEOREM IN SPARSE GRAPHS

with $\Delta(H) \leq \Delta$, and $\mathcal{L}$ be a labelling of vertex set $V(H)$, of bandwidth at most $\beta n$, such that the first $\beta n$ vertices of $\mathcal{L}$ include $C p^{-2}$ vertices that are not contained in any triangles of $H$, and such that there exists a $(k+1)$-colouring that is $(z, \beta)$-zero-free with respect to $\mathcal{L}$, and the colour zero is not assigned to the first $\sqrt{\beta} n$ vertices.

Applying Lemma 26 to $G$, with input $\gamma, k, r_{0}$ and $\varepsilon$, we obtain an integer $r$ with $10 \gamma^{-1} \leq k r \leq r_{1}$, a set $V_{0} \subseteq V(G)$ with $\left|V_{0}\right| \leq C^{*} p^{-2}$, a $k$-equitable partition $\mathcal{V}=\left\{V_{i, j}\right\}_{i \in[r], j \in[k]}$ of $V(G) \backslash V_{0}$, and a graph $R_{r}^{k}$ on vertex set $[r] \times[k]$ with minimum degree $\delta\left(R_{r}^{k}\right) \geq\left(\frac{k-1}{k}+\frac{\gamma}{2}\right) k r$, such that $K_{r}^{k} \subseteq B_{r}^{k} \subseteq R_{r}^{k}$, and such that

(G1a) $\frac{n}{4 k r} \leq\left|V_{i, j}\right| \leq \frac{4 n}{k r}$ for every $i \in[r]$ and $j \in[k]$,

$(G 2 \mathrm{a}) \mathcal{V}$ is $(\varepsilon, d, p)_{G}$-lower-regular on $R_{r}^{k}$ and $(\varepsilon, d, p)_{G}$-super-regular on $K_{r}^{k}$,

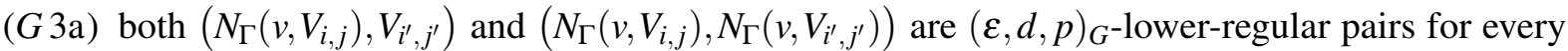
$\left\{(i, j),\left(i^{\prime}, j^{\prime}\right)\right\} \in E\left(R_{r}^{k}\right)$ and $v \in V \backslash V_{0}$, and

(G4a) $\left|N_{\Gamma}\left(v, V_{i, j}\right)\right|=(1 \pm \varepsilon) p\left|V_{i, j}\right|$ for every $i \in[r], j \in[k]$ and every $v \in V \backslash V_{0}$.

Given $i \in[r]$, because $\delta\left(R_{r}^{k}\right)>(k-1) r$, there exists $v \in V\left(R_{r}^{k}\right)$ adjacent to each $(i, j)$ with $j \in[k]$. This, together with our assumptions on $H$, allow us to apply Lemma 27 to $H$, with input $D, k, r, \frac{1}{10} \xi$ and $\beta$, and with $m_{i, j}:=\left|V_{i, j}\right|+\frac{1}{k r}\left|V_{0}\right|$ for each $i \in[r]$ and $j \in[k]$, choosing the rounding such that the $m_{i, j}$ form a $k$-equitable integer partition of $n$. Since $\Delta(H) \leq \Delta$, in particular $H$ is $\Delta$-degenerate. Let $f: V(H) \rightarrow[r] \times[k]$ be the mapping returned by Lemma 27, let $W_{i, j}:=f^{-1}(i, j)$, and let $X \subseteq V(H)$ be the set of special vertices returned by Lemma 27. For every $i \in[r]$ and $j \in[k]$ we have

(H1a) $m_{i, j}-\frac{1}{10} \xi_{n} \leq\left|W_{i, j}\right| \leq m_{i, j}+\frac{1}{10} \xi_{n}$,

(H2a) $|X| \leq \xi n$,

(H3a) $\{f(x), f(y)\} \in E\left(R_{r}^{k}\right)$ for every $\{x, y\} \in E(H)$,

(H 4a) $y, z \in \bigcup_{j^{\prime} \in[k]} f^{-1}\left(i, j^{\prime}\right)$ for every $x \in f^{-1}(i, j) \backslash X$ and $x y, y z \in E(H)$, and

(H 5a) $f(x)=(1, \sigma(x))$ for every $x$ in the first $\sqrt{\beta} n$ vertices of $\mathcal{L}$.

Lemma 27 actually gives a little more, which we do not require for this proof. We let $F$ be the first $\beta n$ vertices of $\mathcal{L}$. By definition of $\mathcal{L}$, in $F$ there are at least $C p^{-2}$ vertices whose neighbourhood in $H$ is independent.

Next, we apply Lemma 22, with input $\varepsilon \mu^{2}$ and $\Delta$, to choose a set $S \subseteq V(G)$ of size $\mu n$. We let the $T_{i}$ of Lemma 22 be all sets which are common neighbourhoods in $\Gamma$ of at most $\Delta$ vertices of $\Gamma$, together with the sets $V_{i, j}$ for $i \in[r]$ and $j \in[k]$. The result of Lemma 22 is that for any $1 \leq \ell \leq \Delta$ and vertices $u_{1}, \ldots, u_{\ell}$ of $V(G)$, we have

$$
\begin{gathered}
\left|S \cap \bigcap_{1 \leq i \leq \ell} N_{\Gamma}\left(u_{i}\right)\right| \\
\left|S \cap V_{i, j}\right| \leq 2 \mu\left|V_{i, j}\right| \quad \text { for each } i \in[r] \text { and } j \in[k],
\end{gathered}
$$

where we use the fact $p \geq C\left(\frac{\log n}{n}\right)^{1 / \Delta}$ and choice of $C$ to deduce $C^{*} \log n<\varepsilon \mu p^{\Delta} n$. 


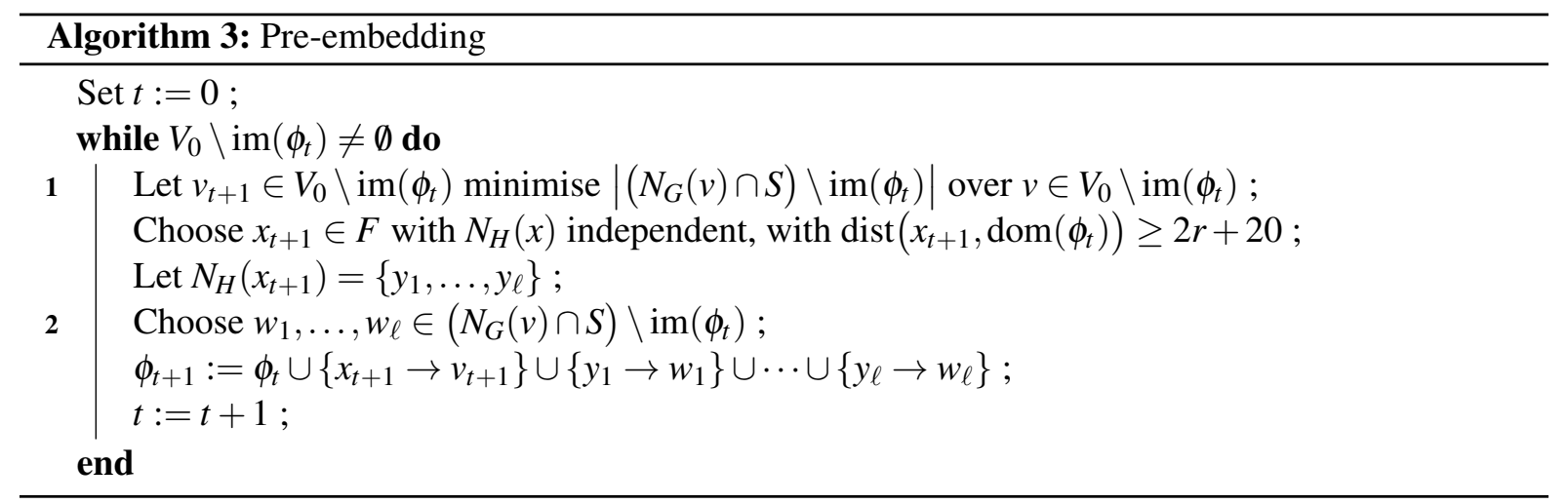

Our next task is to create the pre-embedding that covers the vertices of $V_{0}$. We use the following algorithm, starting with $\phi_{0}$ the empty partial embedding. Suppose this algorithm does not fail, terminating with $t=t^{*}$. The final $\phi_{t^{*}}$ is an embedding of some vertices of $H$ into $V(G)$ which covers $V_{0}$ and is contained in $V_{0} \cup S$. Before we specify how exactly we choose vertices at line 2, we justify that the algorithm does not fail. In other words, we need to justify that at every time $t$ there are vertices of $F$ whose neighbourhood is independent and which are not close to any vertices in $\operatorname{dom}\left(\phi_{t}\right)$, and that at every time $t$, the set $\left(N_{G}(v) \cap S\right) \backslash \operatorname{im}\left(\phi_{t}\right)$ is big. For the first, observe that since $\left|V_{0}\right| \leq C^{*} p^{-2}$, we have $\operatorname{dom}\left(\phi_{t}\right) \leq C^{*} \Delta p^{-2}$ at every step. Thus the number of vertices at distance less than $2 r+20$ from $\operatorname{dom}\left(\phi_{t}\right)$ is at most

$$
\left(1+\Delta+\cdots+\Delta^{2 r+19}\right) C^{*} \Delta p^{-2}<2 C^{*} \Delta^{2 r+20} p^{-2}
$$

which by choice of $C$ is smaller than the number of vertices in $F$ with $N_{H}(x)$ independent. For the second part, suppose that at some time $t$ we pick a vertex $v$ such that $\left|\left(N_{G}(v) \cap S\right) \backslash \operatorname{im}\left(\phi_{t}\right)\right|<\frac{1}{4} \mu$ pn. For each $t-\frac{1}{100(\Delta+1)} \mu p n \leq t^{\prime}<t$, we have $\left|\left(N_{G}(v) \cap S\right) \backslash \operatorname{im}\left(\phi_{t^{\prime}}\right)\right|<\frac{3}{10} \mu p n$, yet at each of these times $v$ is not picked, so that the vertex picked at each time has at most as many uncovered neighbours in $S$ as $v$. Let $Z$ be the set of vertices chosen at line 1 in each of these time steps. Then for each $z \in Z$ we have $\left|\left(N_{G}(v) \cap S\right) \backslash \operatorname{im}\left(\phi_{t}\right)\right| \leq \frac{3}{10} \mu p n$. But since $\delta(G)>\frac{1}{2} p n$, by (8.1) and choice of $\varepsilon$ we have $\left|N_{G}(z) \cap S\right| \geq \frac{2}{5} \mu p n$, so $\left|N_{G}(z) \cap \operatorname{im}\left(\phi_{t}\right)\right| \geq \frac{1}{10} \mu p n$ for each $z \in Z$. By choice of $C$, we have $|Z|=\frac{1}{100(\Delta+1)} \mu p n \geq C^{*} p^{-1} \log n$. Since $|\operatorname{im}(\phi)| \leq(\Delta+1)\left|V_{0}\right| \leq \frac{1}{100} \mu n$, by choice of $C$, this contradicts the good event of Proposition 19.

We have justified that Algorithm 3 completes, and indeed that at each time we reach line 2 there are at least $\frac{1}{4} \mu$ pn vertices of $\left(N_{G}(v) \cap S\right) \backslash \operatorname{im}(\phi)$ to choose from. In order to specify how to choose these vertices, we need the following claim.

Claim 35. Given any set $Y$ of $\frac{1}{4} \mu$ pn vertices of $V(G)$, there exists $W \subseteq Y$ of size at least $\frac{1}{8 r} \mu$ pn and an index $i \in[r]$ with the following property. For each $w \in W$ and each $j \in[k]$, we have $\left|N_{G}\left(w, V_{i, j}\right)\right| \geq d p\left|V_{i, j}\right|$.

Proof. First let $Y^{\prime}$ be obtained from $Y$ by removing all vertices $y \in Y$ such that either $\left|N_{\Gamma}\left(y, V_{0}\right)\right| \geq \varepsilon$ p , or for some $i \in[r]$ and $j \in[k]$ we have $\left|N_{\Gamma}\left(y, V_{i, j}\right)\right| \neq(1 \pm \varepsilon) p\left|V_{i, j}\right|$. Because the good event of Proposition 19 occurs, the total number of vertices removed is at most $2 k r C^{*} p^{-1} \log n<\frac{1}{2}|Y|$, where the inequality is by choice of $C$. Now given any $y \in Y^{\prime}$, if for each $i \in[r]$ there is $j \in[k]$ such that $\left|N_{G}\left(y, V_{i, j}\right)\right|<d p\left|V_{i, j}\right|$, 
The BANDWIDTH THEOREM IN SPARSE GRAPHS

then, since the $\left\{V_{i, j}\right\}$ are $k$-equitable, we have $\left|N_{G}(y)\right| \leq \varepsilon p n+d p n+(1+\varepsilon) \frac{k-1}{k} p n+r<\left(\frac{k-1}{k}+\gamma\right) p n$, a contradiction. We conclude that for each $y \in Y^{\prime}$ there exists $i \in[r]$ such that $\left|N_{G}\left(y, V_{i, j}\right)\right| \geq d p\left|V_{i, j}\right|$ for each $j \in[k]$. We let $W$ be the vertices of $Y^{\prime}$ giving a majority choice of $i$.

Now at each time $t$, in line 2 of Algorithm 3, we choose the vertices $w_{1}, \ldots, w_{\ell}$ as follows. Let $Y=\left(N_{G}\left(v_{t}\right) \cap S\right) \backslash \operatorname{im}\left(\phi_{t}\right)$. Let $i_{t} \in[r]$ be an index, and $W \subseteq Y$ be a set of size $\frac{1}{8 r} \mu p n$, such that $\left|N_{G}\left(w, V_{i_{t}, j}\right)\right| \geq d p n\left|V_{i_{t}, j}\right|$ for each $j \in[k]$, whose existence is guaranteed by Claim 35. By construction, and by our choice of $\mu$, we can apply Lemma 28 with input $d, k, \Delta, \varepsilon^{*}, r$ and $\varepsilon$, with the clusters $\left\{V_{i_{t}, j}\right\}_{j \in[k]}$ as the $\left\{V_{i}\right\}_{i \in[k]}$, and inputting a subset of $W$ of size $10^{-10} \frac{\varepsilon^{4} p n}{k^{4} r^{4}}$ as requried for (V3). This last is possible by choice of $\mu$. To verify the conditions of Lemma 28, observe that (V1) follows from (G1a), (V2) from $(G 2 \mathrm{a})$, and $(V 4)$ from Claim 35 . We obtain a $\Delta$-tuple of vertices in $W$ satisfying $(W 1)-(W 4)$. We let $w_{1}, \ldots, w_{\ell}$ be the first $\ell$ vertices of this tuple.

Let $H^{\prime}=H-\operatorname{dom}\left(\phi_{t^{*}}\right)$. We next define image restricting vertex sets and create an updated homomorphism $f^{*}: V\left(H^{\prime}\right) \rightarrow[r] \times[k]$. For each $x \in V(H) \backslash \operatorname{dom}\left(\phi_{t^{*}}\right)$, let $J_{x}=\phi_{t^{*}}\left(N_{H}(x) \cap \operatorname{dom}\left(\phi_{t^{*}}\right)\right)$. Now, since the vertices $\left\{x_{t}\right\}_{t \in\left[t^{*}\right]}$ are by construction at pairwise distance at least $2 r+20$, in particular for each $y \in V\left(H^{\prime}\right)$ with $J_{y} \neq \emptyset$ the vertex $y$ is at distance two from one $x_{t}$, and at distance greater than $r+10$ from all others. Let $j \in[k]$ such that $f(y)=(1, j)$. Then we set $f^{*}(y):=\left(i_{t}, j\right)$. Next, for each $t \in\left[t^{*}\right]$ and each $z \in V(H)$ at distance $3, \ldots, i_{t}+1$ from $x_{t}$, we set $f^{*}(z)$ as follows. Recall that $f(z)=(1, j)$ for some $j \in[k]$. We set $f^{*}(z)=\left(i_{t}+2-\operatorname{dist}\left(x_{t}, z\right), j\right)$. Because the $\left\{x_{t}\right\}$ are at pairwise distance at least $2 r+20$, no vertex is at distance $r+5$ or less from any two $x_{t}$ and $x_{t^{\prime}}$, so that $f^{*}$ is well-defined. Because $R_{r}^{k}$ contains $B_{r}^{k}$, the $f^{*}$ we constructed so far is a graph homomorphism. Furthermore, for each $x_{t}$ the set of vertices $z$ at distance $i_{t}+1$ from $x_{t}$ are in the first $\sqrt{\beta} n$ vertices of $\mathcal{L}$, and so by $(H 5 \mathrm{a})$ satisfy $f^{*}(z)=f(z)$. We complete the construction of $f^{*}$ by setting $f^{*}(z)=f(z)$ for each remaining $z \in V(H) \backslash \operatorname{dom}\left(\phi_{t^{*}}\right)$. Because $f$ is a graph homomorphism, $f^{*}$ is also a graph homomorphism whose domain is $V\left(H^{\prime}\right)$. For each $i \in[r]$ and $j \in[k]$, let $W_{i, j}^{\prime}$ be the set of vertices $w \in V\left(H^{\prime}\right)$ with $f^{*}(w) \in V_{i, j}$, and let $X^{\prime}$ consist of $X$ together with all vertices of $H^{\prime}$ at distance $r+10$ or less from some $x_{t}$ with $t \in\left[t^{*}\right]$. The total number of vertices $z \in V(H)$ at distance at most $r+10$ from some $x_{t}$ is at most $2 \Delta^{r+10}\left|V_{0}\right|<\frac{1}{100} \xi n$. Since $W_{i, j} \triangle W_{i, j}^{\prime}$ contains only such vertices, we have

(H1b) $m_{i, j}-\frac{1}{5} \xi_{n} \leq\left|W_{i, j}^{\prime}\right| \leq m_{i, j}+\frac{1}{5} \xi_{n}$,

(H2b) $\left|X^{\prime}\right| \leq 2 \xi n$,

(H3b) $\left\{f^{*}(x), f^{*}(y)\right\} \in E\left(R_{r}^{k}\right)$ for every $\{x, y\} \in E\left(H^{\prime}\right)$, and

(H4b) $y, z \in \bigcup_{j^{\prime} \in[k]} W_{i, j^{\prime}}^{\prime}$ for every $x \in W_{i, j}^{\prime} \backslash X^{\prime}$ and $x y, y z \in E\left(H^{\prime}\right)$.

where $(H 2 \mathrm{~b}),(H 3 \mathrm{~b})$ and $(H 4 \mathrm{~b})$ hold by $(H 2 \mathrm{a})$ and definition of $X^{\prime}$, by definition of $f^{*}$, and by $(H 4 \mathrm{a})$ and choice of $X^{\prime}$ respectively.

Furthermore, we have

(G1a) $\frac{n}{4 k r} \leq\left|V_{i, j}\right| \leq \frac{4 n}{k r}$ for every $i \in[r]$ and $j \in[k]$,

$\left(G\right.$ a) $\mathcal{V}$ is $(\varepsilon, d, p)_{G}$-lower-regular on $R_{r}^{k}$ and $(\varepsilon, d, p)_{G}$-super-regular on $K_{r}^{k}$, 
(G3a) both $\left(N_{\Gamma}\left(v, V_{i, j}\right), V_{i^{\prime}, j^{\prime}}\right)$ and $\left(N_{\Gamma}\left(v, V_{i, j}\right), N_{\Gamma}\left(v, V_{i^{\prime}, j^{\prime}}\right)\right)$ are $(\varepsilon, d, p)_{G}$-lower-regular pairs for every $\left\{(i, j),\left(i^{\prime}, j^{\prime}\right)\right\} \in E\left(R_{r}^{k}\right)$ and $v \in V \backslash V_{0}$, and

(G4a) $\left|N_{\Gamma}\left(v, V_{i, j}\right)\right|=(1 \pm \varepsilon) p\left|V_{i, j}\right|$ for every $i \in[r], j \in[k]$ and every $v \in V \backslash V_{0}$.

(G 5a) $\left|V_{f^{*}(x)} \cap \bigcap_{u \in J_{x}} N_{G}(u)\right| \geq \alpha p^{\left|J_{x}\right|}\left|V_{f^{*}(x)}\right|$ for each $x \in V\left(H^{\prime}\right)$,

(G 6a) $\left|V_{f^{*}(x)} \cap \bigcap_{u \in J_{x}} N_{\Gamma}(u)\right|=\left(1 \pm \varepsilon^{*}\right) p^{\left|J_{x}\right|}\left|V_{f^{*}(x)}\right|$ for each $x \in V\left(H^{\prime}\right)$, and

(G 7a) $\left(V_{f^{*}(x)} \cap \bigcap_{u \in J_{x}} N_{\Gamma}(u), V_{f^{*}(y)} \cap \bigcap_{v \in J_{y}} N_{\Gamma}(v)\right)$ is $\left(\varepsilon^{*}, d, p\right)_{G^{-}}$lower-regular for each $x y \in E\left(H^{\prime}\right)$.

(G 8a) $\left|\bigcap_{u \in J_{x}} N_{\Gamma}(u)\right| \leq\left(1+\varepsilon^{*}\right) p^{\left|J_{x}\right|} n$ for each $x \in V\left(H^{\prime}\right)$,

Properties $(G 1 \mathrm{a})$ to $(G 4 \mathrm{a})$ are repeated for convenience. Properties $(G 5 \mathrm{a}),(G 6 \mathrm{a})$ and $(G 8 \mathrm{a})$, are trivial when $J_{x}=\emptyset$, and are otherwise guaranteed by Lemma 28. Finally $(G 7 \mathrm{a})$ follows from $(G 2 \mathrm{a})$ when $J_{x}, J_{y}=\emptyset$, and otherwise is guaranteed by Lemma 28 .

For each $i \in[r]$ and $j \in[k]$, let $V_{i, j}^{\prime}=V_{i, j} \backslash \operatorname{im}\left(\phi_{t^{*}}\right)$, and let $\mathcal{V}^{\prime}=\left\{V_{i, j}^{\prime}\right\}_{i \in[r], j \in[k]}$. Because $V_{i, j} \backslash V_{i, j}^{\prime} \subseteq S$ for each $i \in[r]$ and $j \in[k]$, using (8.1) and Proposition 12, and our choice of $\mu$, we obtain

(G1b) $\frac{n}{6 k r} \leq\left|V_{i, j}^{\prime}\right| \leq \frac{6 n}{k r}$ for every $i \in[r]$ and $j \in[k]$,

$(G 2 \mathrm{~b}) \mathcal{V}^{\prime}$ is $(2 \varepsilon, d, p)_{G}$-lower-regular on $R_{r}^{k}$ and $(2 \varepsilon, d, p)_{G}$-super-regular on $K_{r}^{k}$,

(G 3b) both $\left(N_{\Gamma}\left(v, V_{i, j}^{\prime}\right), V_{i^{\prime}, j^{\prime}}^{\prime}\right)$ and $\left(N_{\Gamma}\left(v, V_{i, j}^{\prime}\right), N_{\Gamma}\left(v, V_{i^{\prime}, j^{\prime}}^{\prime}\right)\right)$ are $(2 \varepsilon, d, p)_{G^{-}}$-lower-regular pairs for every $\left\{(i, j),\left(i^{\prime}, j^{\prime}\right)\right\} \in E\left(R_{r}^{k}\right)$ and $v \in V \backslash V_{0}$, and

(G 4b) $\left|N_{\Gamma}\left(v, V_{i, j}^{\prime}\right)\right|=(1 \pm 2 \varepsilon) p\left|V_{i, j}\right|$ for every $i \in[r], j \in[k]$ and every $v \in V \backslash V_{0}$.

(G 5b) $\left|V_{f^{*}(x)}^{\prime} \cap \bigcap_{u \in J_{x}} N_{G}(u)\right| \geq \frac{1}{2} \alpha p^{\left|J_{x}\right|}\left|V_{f^{*}(x)}^{\prime}\right|$,

(G 6b) $\left|V_{f^{*}(x)}^{\prime} \cap \bigcap_{u \in J_{x}} N_{\Gamma}(u)\right|=\left(1 \pm 2 \varepsilon^{*}\right) p^{\left|J_{x}\right|}\left|V_{f^{*}(x)}^{\prime}\right|$, and

(G 7b) $\left(V_{f^{*}(x)}^{\prime} \cap \bigcap_{u \in J_{x}} N_{\Gamma}(u), V_{f^{*}(y)}^{\prime} \cap \bigcap_{v \in J_{y}} N_{\Gamma}(v)\right)$ is $\left(2 \varepsilon^{*}, d, p\right)_{G^{-}}$-lower-regular.

(G8b) $\left|\bigcap_{u \in J_{x}} N_{\Gamma}(u)\right| \leq\left(1+2 \varepsilon^{*}\right) p^{\left|J_{x}\right|} n$ for each $x \in V\left(H^{\prime}\right)$,

We are now almost finished. The only remaining problem is that we do not necessarily have $\left|W_{i, j}^{\prime}\right|=\left|V_{i, j}^{\prime}\right|$ for each $i \in[r]$ and $j \in[k]$. Since $\left|V_{i, j}^{\prime}\right|=\left|V_{i, j}\right| \pm 2 \Delta^{r+10}\left|V_{0}\right|=m_{i, j} \pm 3 \Delta^{r+10}\left|V_{0}\right|$, by $(H 1 \mathrm{~b})$ we have $\left|V_{i, j}^{\prime}\right|=\left|W_{i, j}^{\prime}\right| \pm \xi n$. We can thus apply Lemma 29, with input $k, r_{1}, \Delta, \gamma, d, 8 \varepsilon$, and $r$. This gives us sets $V_{i, j}^{\prime \prime}$ with $\left|V_{i, j}^{\prime \prime}\right|=\left|W_{i, j}^{\prime}\right|$ for each $i \in[r]$ and $j \in[k]$ by $\left(B 1^{\prime}\right)$. Let $\mathcal{V}^{\prime \prime}=\left\{V_{i, j}^{\prime \prime}\right\}_{i \in[r], j \in[k]}$. Lemma 29 guarantees us the following.

(G1c) $\frac{n}{8 k r} \leq\left|V_{i, j}^{\prime \prime}\right| \leq \frac{8 n}{k r}$ for every $i \in[r]$ and $j \in[k]$,

$(G 2 \mathrm{c}) \mathcal{V}^{\prime \prime}$ is $\left(4 \varepsilon^{*}, d, p\right)_{G}$-lower-regular on $R_{r}^{k}$ and $\left(4 \varepsilon^{*}, d, p\right)_{G^{-}}$-super-regular on $K_{r}^{k}$,

$(G 3 \mathrm{c})$ both $\left(N_{\Gamma}\left(v, V_{i, j}^{\prime \prime}\right), V_{i^{\prime}, j^{\prime}}^{\prime \prime}\right)$ and $\left(N_{\Gamma}\left(v, V_{i, j}^{\prime \prime}\right), N_{\Gamma}\left(v, V_{i^{\prime}, j^{\prime}}^{\prime \prime}\right)\right)$ are $\left(4 \varepsilon^{*}, d, p\right)_{G^{-}}$-lower-regular pairs for every $\left\{(i, j),\left(i^{\prime}, j^{\prime}\right)\right\} \in E\left(R_{r}^{k}\right)$ and $v \in V \backslash V_{0}$, and 
(G4c) we have $(1-4 \varepsilon) p\left|V_{i, j}^{\prime \prime}\right| \leq\left|N_{\Gamma}\left(v, V_{i, j}^{\prime \prime}\right)\right| \leq(1+4 \varepsilon) p\left|V_{i, j}^{\prime \prime}\right|$ for every $i \in[r], j \in[k]$ and every $v \in$ $V \backslash V_{0}$.

$(G 5 \mathrm{c})\left|V_{f^{*}(x)}^{\prime \prime} \cap \bigcap_{u \in J_{x}} N_{G}(u)\right| \geq \frac{1}{4} \alpha p^{\left|J_{x}\right|}\left|V_{f^{*}(x)}^{\prime \prime}\right|$,

(G6c) $\left|V_{f^{*}(x)}^{\prime \prime} \cap \bigcap_{u \in J_{x}} N_{\Gamma}(u)\right|=\left(1 \pm 4 \varepsilon^{*}\right) p^{\left|J_{x}\right|}\left|V_{f^{*}(x)}^{\prime}\right|$, and

$(G 7 \mathrm{c})\left(V_{f^{*}(x)}^{\prime \prime} \cap \bigcap_{u \in J_{x}} N_{\Gamma}(u), V_{f^{*}(y)}^{\prime \prime} \cap \bigcap_{v \in J_{y}} N_{\Gamma}(v)\right)$ is $\left(4 \varepsilon^{*}, d, p\right)_{G}$-lower-regular.

Here $(G 1 \mathrm{c})$ comes from $(G 1 \mathrm{~b})$ and $\left(B 2^{\prime}\right)$, while $(G 2 \mathrm{c})$ comes from $\left(B 3^{\prime}\right)$ and choice of $\varepsilon$. $(G 3 \mathrm{c})$ is guaranteed by $\left(B 4^{\prime}\right)$. Now, each of $(G 4 \mathrm{c}),(G 5 \mathrm{c})$ and $(G 6 \mathrm{c})$ comes from the corresponding $(G 4 \mathrm{~b}),(G 5 \mathrm{~b})$ and $(G 6 \mathrm{~b})$ together with $\left(B 5^{\prime}\right)$. Finally, $(G 7 \mathrm{c})$ comes from $(G 7 \mathrm{~b})$ and $(G 8 \mathrm{~b})$ together with Proposition 12 and $\left(B 5^{\prime}\right)$.

For each $x \in V\left(H^{\prime}\right)$ with $J_{x}=\emptyset$, let $I_{x}=V_{f^{*}(x)}^{\prime \prime}$. For each $x \in V\left(H^{\prime}\right)$ with $J_{x} \neq \emptyset$, let $I_{x}=V_{f^{*}(x)}^{\prime \prime} \cap$ $\bigcap_{u \in J_{x}} N_{G}(u)$. Now $\mathcal{W}^{\prime}$ and $\mathcal{V}^{\prime \prime}$ are $\kappa$-balanced by $(G 1 \mathrm{c})$, size-compatible by construction, partitions of respectively $V\left(H^{\prime}\right)$ and $V(G) \backslash \operatorname{im}\left(\phi_{t^{*}}\right)$, with parts of size at least $n /\left(\kappa r_{1}\right)$ by $(G 1 \mathrm{c})$. Letting $\widetilde{W}_{i, j}:=$ $W_{i, j}^{\prime} \backslash X^{\prime}$, by (H2b), choice of $\xi$, and $(H 4 \mathrm{~b}),\left\{\widetilde{W}_{i, j}\right\}_{i \in[r], j \in[k]}$ is a $\left(\vartheta, K_{r}^{k}\right)$-buffer for $H^{\prime}$. Furthermore since $f^{*}$ is a graph homomorphism from $H^{\prime}$ to $R_{r}^{k}$, we have (BUL1). By $(G 2 \mathrm{c}),(G 3 \mathrm{c})$ and $(G 4 \mathrm{c})$ we have (BUL2), with $R=R_{r}^{k}$ and $R^{\prime}=K_{r}^{k}$. Finally, the pair (J, J) $=\left(\left\{I_{x}\right\}_{x \in V\left(H^{\prime}\right)},\left\{J_{x}\right\}_{x \in V\left(H^{\prime}\right)}\right)$ form a $\left(\rho, \frac{1}{4} \alpha, \Delta, \Delta\right)$-restriction pair. To see this, observe that the total number of image restricted vertices in $H^{\prime}$ is at most $\Delta^{2}\left|V_{0}\right|<\rho\left|V_{i, j}\right|$ for any $i \in[r]$ and $j \in[k]$, giving (RP 1). Since for each $x \in V\left(H^{\prime}\right)$ we have $\left|J_{x}\right|+\operatorname{deg}_{H^{\prime}}(x)=\operatorname{deg}_{H}(x) \leq \Delta$ we have (RP 3), while (RP 2) follows from (G 5c), and (RP 5) follows from $(G 6 \mathrm{c})$. Finally, $(R P 6)$ follows from $(G 7 \mathrm{c})$, and $(R P 4)$ follows since $\Delta(H) \leq \Delta$. Together this gives (BUL 3). Thus, by Lemma 16 there exists an embedding $\phi$ of $H^{\prime}$ into $G \backslash \operatorname{im}\left(\phi_{t^{*}}\right)$, such that $\phi(x) \in I_{x}$ for each $x \in V\left(H^{\prime}\right)$. Finally, $\phi \cup \phi_{t^{*}}$ is an embedding of $H$ in $G$, as desired.

With Theorem 25 in hand, we can now present the proof of Theorem 7.

Proof of Theorem 7. Given $\gamma, \Delta$, and $k$, let $\beta>0, z>0$, and $C>0$ be returned by Theorem 25 with input $\gamma, \Delta$, and $k$. Set $\beta^{*}:=\beta / 2$ and $C^{*}:=C / \beta$. Let $H$ be a $k$-colourable graph on $n$ vertices with $\Delta(H) \leq \Delta$ such that there exists a set $W$ of at least $C^{*} p^{-2}$ vertices in $V(H)$ that are not contained in any triangles of $H$ and such that there exists a labelling $\mathcal{L}$ of its vertex set of bandwidth at most $\beta^{*} n$. By the choice of $C^{*}$ we find an interval $I \subseteq \mathcal{L}$ of length $\beta n$ containing a subset $F \subseteq W$ with $|F|=C p^{-2}$. Now we can rearrange the labelling $\mathcal{L}$ to a labelling $\mathcal{L}^{\prime}$ of bandwidth at most $2 \beta^{*} n=\beta n$ such that $F$ is contained in the first $\beta n$ vertices in $\mathcal{L}^{\prime}$. Then, by Theorem 25 we know that $\Gamma=G(n, p)$ satisfies the following a.a.s. if $p \geq C(\log n / n)^{1 / \Delta}$ and in particular if $p \geq C^{*}(\log n / n)^{1 / \Delta}$. If $G$ is a spanning subgraph of $\Gamma$ with $\delta(G) \geq((k-1) / k+\gamma) p n$, then $G$ contains a copy of $H$, which finishes the proof.

\section{Lowering the probability for degenerate graphs}

As with Theorem 7, we deduce Theorem 8 from the following more general statement. 
Theorem 36. For each $\gamma>0, \Delta \geq 2, D \geq 1$ and $k \geq 1$, there exist constants $\beta>0, z>0$, and $C>0$ such that the following holds asymptotically almost surely for $\Gamma=G(n, p)$ if $p \geq C\left(\frac{\log n}{n}\right)^{1 /(2 D+1)}$. Let $G$ be a spanning subgraph of $\Gamma$ with $\delta(G) \geq\left(\frac{k-1}{k}+\gamma\right)$ pn and let $H$ be a graph on $n$ vertices with $\Delta(H) \leq \Delta$ and degeneracy at most $D$, that has a labelling $\mathcal{L}$ of its vertex set of bandwidth at most $\beta n, a(k+1)$-colouring that is $(z, \beta)$-zero-free with respect to $\mathcal{L}$ and where the first $\sqrt{\beta}$ n vertices in $\mathcal{L}$ are not given colour zero and the first $\beta n$ vertices in $\mathcal{L}$ include $C p^{-2}$ vertices that are not in any triangles or copies of $C_{4}$ in $H$. Then $G$ contains a copy of $H$.

The proof of Theorem 36 is quite similar to that of Theorem 25 . We provide only a sketch, highlighting the differences. (For more details and background on this result see [22].) The most important of these differences are that we do not use Lemma 28 in the pre-embedding, and that we use a version of Lemma 16 whose performance is better for degenerate graphs. In order to state this, we need the following definitions. Given an order $\tau$ on $V(H)$ and a family $\mathcal{J}$ of image restricting vertices, we define $\pi^{\tau}(x):=\left|J_{x}\right|+\left|\left\{y \in N_{H}(x): \tau(y)<\tau(x)\right\}\right|$. Now the condition on $\tau$ we need for our enhanced blow-up lemma is the following.

Definition $37((\tilde{D}, p, m)$-bounded order). Let $H$ be a graph given with buffer sets $\widetilde{\mathcal{W}}$ and a restriction pair $\mathcal{J}=\left\{I_{i}\right\}_{i \in[r]}$ and $\mathcal{J}=\left\{J_{i}\right\}_{i \in[r]}$. Let $\widetilde{W}=\bigcup \widetilde{\mathcal{W}}$. Let $\tau$ be an ordering of $V(H)$ and $W^{e} \subseteq V(H)$. Then $\tau$ is a $(\tilde{D}, p, m)$-bounded order for $H, \widetilde{\mathcal{W}}, \mathcal{J}$ and $\mathcal{J}$ with exceptional set $W^{e}$ if the following conditions are satisfied for each $x \in V(H)$.

(ORD 1) Define

$$
\tilde{D}_{x}:= \begin{cases}\tilde{D}-2 & \text { if there is } y z \in E(H) \text { with } y, z \in N_{H}(x) \text { and } \tau(y), \tau(z)>\tau(x) \\ \tilde{D}-1 & \text { else if there is } y \in N_{H}(x) \text { with } \tau(y)>\tau(x) \\ \tilde{D} & \text { otherwise. }\end{cases}
$$

We have $\pi^{\tau}(x) \leq \tilde{D}_{x}$, and if $x \in N(\widetilde{W})$ even $\pi^{\tau}(x) \leq \tilde{D}_{x}-1$. Finally, if $x \in \widetilde{W}$ we have $\operatorname{deg}(x) \leq \tilde{D}$.

(ORD 2) One of the following holds:

- $x \in W^{e}$,

- $\pi^{\tau}(x) \leq \frac{1}{2} \tilde{D}$

- $x$ is not image restricted and every neighbour $y$ of $x$ with $\tau(y)<\tau(x)$ satisfies $\tau(x)-\tau(y) \leq$ $p^{\pi^{\tau}(x)} m$.

(ORD 3) If $x \in N(\widetilde{W})$ then all but at most $\tilde{D}-1-\max _{z \notin W^{e}} \pi^{\tau}(z)$ neighbours $y$ of $x$ with $\tau(y)<\tau(x)$ satisfy $\tau(x)-\tau(y) \leq p^{\tilde{D}} m$.

To obtain the best probability bound, one should choose $\tau$ to minimise $\tilde{D}$. In the proof of Theorem 36 we will take $\tau$ to be an order witnessing $D$-degeneracy, $W^{e}$ will contain all image restricted vertices, and we will choose buffer sets containing vertices of degree at most $2 D+1$. One can easily check that this allows us to choose $\tilde{D}=2 D+1$. 
Lemma 38 ([2, Lemma 1.23]). For all $\Delta \geq 2, \Delta_{R^{\prime}}, \Delta_{J}, \tilde{D}, \alpha, \zeta, d>0, \kappa>1$ there exist $\varepsilon, \rho>0$ such that for all $r_{1}$ there is a $C$ such that for

$$
p \geq C\left(\frac{\log n}{n}\right)^{1 / \tilde{D}}
$$

the random graph $\Gamma=G_{n, p}$ a.a.s. satisfies the following.

Let $R$ be a graph on $r \leq r_{1}$ vertices and let $R^{\prime} \subseteq R$ be a spanning subgraph with $\Delta\left(R^{\prime}\right) \leq \Delta_{R^{\prime}}$. Let $H$ and $G \subseteq \Gamma$ be graphs with $\kappa$-balanced, size-compatible vertex partitions $\mathcal{W}=\left\{W_{i}\right\}_{i \in[r]}$ and $\mathcal{V}=\left\{V_{i}\right\}_{i \in[r]}$, respectively, which have parts of size at least $m \geq n /\left(\kappa r_{1}\right)$. Let $\widetilde{\mathcal{W}}=\left\{\widetilde{W}_{i}\right\}_{i \in[r]}$ be a family of subsets of $V(H), \mathcal{J}=\left\{I_{x}\right\}_{x \in V(H)}$ be a family of image restrictions, and $\mathcal{J}=\left\{J_{x}\right\}_{x \in V(H)}$ be a family of restricting vertices. Let $\tau$ be an order of $V(H)$ and $W^{e} \subseteq V(H)$ be a set of size $\left|W^{e}\right| \leq \varepsilon p^{\max _{x \in W^{e}} \pi^{\tau}(x)} n / r_{1}$. Suppose that

(DBUL 1) $\Delta(H) \leq \Delta,(H, \mathcal{W})$ is an R-partition, and $\widetilde{\mathcal{W}}$ is an $\left(\alpha, R^{\prime}\right)$-buffer for $H$,

(DBUL2) $(G, \mathcal{V})$ is an $(\varepsilon, d, p)$-lower-regular $R$-partition, which is $(\varepsilon, d, p)$-super-regular on $R^{\prime}$, has one-sided inheritance on $R^{\prime}$, and two-sided inheritance on $R^{\prime}$ for $\widetilde{\mathcal{W}}$,

(DBUL 3) J and J form a $\left(\rho, \zeta, \Delta, \Delta_{J}\right)$-restriction pair.

$(D B U L 4) \tau$ is a $\left(\tilde{D}, p, \varepsilon n / r_{1}\right)$-bounded order for $H, \widetilde{\mathcal{W}}, \mathcal{J}$, J with exceptional set $W^{e}$.

Then there is an embedding $\psi: V(H) \rightarrow V(G)$ such that $\psi(x) \in I_{x}$ for each $x \in H$.

Sketch proof of Theorem 36. We set up constants quite similarly as in the proof of Theorem 25. Specifically, given $\gamma>0, \Delta \geq 2, D$ and $k \geq 2$, let $d$ be returned by Lemma 26, with input $\gamma, k$ and $r_{0}:=10 \gamma^{-1}$. Let $\alpha=\frac{d}{2}$. Let $\tilde{D}=2 D+1$. Now let $\varepsilon_{\mathrm{BL}}>0$ and $\rho>0$ be returned by Lemma 38 with input $\Delta, \Delta_{R^{\prime}}=3 k$, $\Delta_{J}=\Delta, \tilde{D}^{\prime}, \vartheta=\frac{1}{100 D}, \zeta=\frac{1}{4} \alpha, d$ and $\kappa=64$. Let $\varepsilon^{*}=\frac{1}{8} \varepsilon_{\mathrm{BL}}$, and then Lemma 17 , for input $\varepsilon^{*}$ and $d$, returns $\varepsilon_{1}>0$. Let $\varepsilon_{0}>0$ be small enough both for Lemma 18 with input $\varepsilon^{*}$ and $d$, and for Lemma 17 with input $\varepsilon_{1}$ and $d$.

We choose $\varepsilon=\min \left(\varepsilon_{0}, d, \frac{1}{4 D} \varepsilon^{*}, \frac{1}{2 k}\right)$. Putting $\varepsilon$ into Lemma 26 returns $r_{1}$. Next, Lemma 29, for input $k, r_{1}, \Delta, \gamma, d$ and $8 \varepsilon$, returns $\xi>0$. We assume without loss of generality that $\xi \leq 1 /\left(10 k r_{1}\right)$, and set $\beta=10^{-12} \xi^{2} /\left(\Delta k^{4} r_{1}^{2}\right)$. Let $\mu=\frac{\varepsilon^{2}}{100000 k r}$. Finally, suppose $C^{*}$ is large enough for each of these lemmas, for Lemma 16, for Proposition 19 with input $\varepsilon$, and for Lemma 22 with input $\varepsilon \mu^{2}$ and $\Delta$.

We set $C=10^{10} k^{2} r_{1}^{2} \varepsilon^{-2} \xi^{-1} \Delta^{2 r_{1}+20} \mu^{-1} C^{*}$, and $z=10 / \xi$. Given $p \geq C\left(\frac{\log n}{n}\right)^{1 /(2 D+1)}$, a.a.s. $G(n, p)$ satisfies the good events of Lemma 38, Lemma 26, Lemma 17 and Lemma 18, and Proposition 19, with the stated inputs. Suppose that $\Gamma=G(n, p)$ satisfies these good events.

Let $G$ be a spanning subgraph of $\Gamma$ with $\delta(G) \geq\left(\frac{k-1}{k}+\gamma\right) p n$. Let $H$ be any graph on $n$ vertices with $\Delta(H) \leq \Delta$, and let $\mathcal{L}$ be a labelling of $V(H)$ of bandwidth at most $\beta n$ whose first $\beta n$ vertices include $C p^{-2}$ vertices that are not contained in any triangles or four-cycles of $H$, and such that there exists a $(k+1)$-colouring that is $(z, \beta)$-zero-free with respect to $\mathcal{L}$, and the colour zero is not assigned to the first $\sqrt{\beta} n$ vertices. Furthermore, let $\tau$ be a $D$-degeneracy order of $V(H)$.

Next, as in the proof of Theorem 25, we apply Lemma 26 to $G$, obtaining a partition of $V(G)$ with the properties $(G 1 \mathrm{a})-(G 4 \mathrm{a})$. Note that if $D=1$, in place of (G3a) we will ask only for the weaker condition 


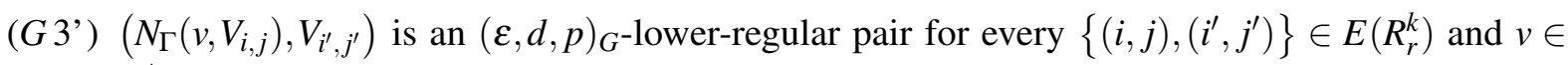
$V \backslash V_{0}$,

and thus for $D=1$ we have $\left|V_{0}\right| \leq C^{*} p^{-1}$, while for $D \geq 2$ we have $\left|V_{0}\right| \leq C^{*} p^{-2}$.

Next, we apply Lemma 27 to obtain a partition of $V(H)$. We use the same inputs as in the proof of Theorem 25, with the exception that $D$ is now given in the statement of Theorem 36 rather than being set equal to $\Delta$. The result is a function $f: V(H) \rightarrow V\left(R_{r}^{k}\right)$ and a special set $X$ with the same properties $(H 1 \mathrm{a})-(\mathrm{H} 5 \mathrm{a})$, and in addition

(H6a) $\left|\left\{x \in f^{-1}(i, j): \operatorname{deg}(x) \leq 2 D\right\}\right| \geq \frac{1}{24 D}\left|f^{-1}(i, j)\right|$.

We now continue following the proof of Theorem 25, using Lemma 22 with input $\varepsilon \mu^{2}$ and $D+1$ (rather than $\varepsilon \mu^{2}$ and $\Delta$ ), to choose a set $S$ satisfying (8.1) for each $1 \leq \ell \leq D+1$ and vertices $u_{1}, \ldots, u_{\ell}$ of $V(G)$. We use the same pre-embedding Algorithm 3, with the exception that we choose vertices at line 2 differently. As before, given $v_{t+1} \in V_{0} \backslash \operatorname{im}\left(\phi_{t}\right)$, we use Claim 35 to find a set $W \subseteq N_{G}\left(v_{t+1}\right)$ of size at least $\frac{1}{8 r} \mu p n$ and an index $i \in[r]$ such that for each $w \in W$ we have $\left|N_{G}\left(w, V_{i, j}\right)\right| \geq d p\left|V_{i, j}\right|$ for each $j \in[k]$. However, rather than applying Lemma 28, we let $w_{1}, \ldots, w_{\ell}$ be distinct vertices of $W$ which satisfy $(G 5 \mathrm{a})-(G 8 \mathrm{a})$. We now justify that this is possible. We choose the $w_{1}, \ldots, w_{\ell}$ successively. Since $x_{t+1}$ is not contained in any triangle or four-cycle of $H$, we have $\left|J_{x}\right| \leq 1$ for each $x \in V(H)$, so that $(G 5 \mathrm{a})$ is automatically satisfied. By Proposition 19, $(G 6 \mathrm{a})$ and $(G 8 \mathrm{a})$ are satisfied for all but at most $2 C^{*} k r_{1} p^{-1} \log n$ vertices of $W$. It remains to show that we can obtain $(G 7 \mathrm{a})$, which we do as follows. For $s \in[\ell]$, when we come to choose $w_{s}$, we insist that for any $\left\{(i, j),\left(i^{\prime}, j^{\prime}\right)\right\} \in E\left(R_{r}^{k}\right)$, the following hold. First, $\left(N_{\Gamma}\left(w_{s}, V_{i, j}\right), V_{i^{\prime}, j^{\prime}}\right)$ is $\left(\varepsilon_{1}, d, p\right)_{G^{-}}$lower-regular. Second, $\left(N_{\Gamma}\left(w_{s}, V_{i, j}\right), N_{\Gamma}\left(w_{s}, V_{i^{\prime}, j^{\prime}}\right)\right)$ is $\left(\varepsilon^{*}, d, p\right)_{G^{-}}$ lower-regular. Third, for each $1 \leq t \leq s-1,\left(N_{\Gamma}\left(w_{s}, V_{i, j}\right), N_{\Gamma}\left(w_{t}, V_{i^{\prime}, j^{\prime}}\right)\right)$ is $\left(\varepsilon^{*}, d, p\right)_{G^{\prime}}$-lower-regular. The conditions of respectively Lemma 17, Lemma 18, and Lemma 17 are in each case satisfied (in the last case by choice of $w_{t}$ ) and thus in total at most $3 C^{*} k^{2} r_{1}^{2} \max \left\{p^{-2}, p^{-1} \log n\right\}$ vertices of $W$ are prohibited. Since $5 C^{*} k^{2} r_{1}^{2} \max \left\{p^{-2}, p^{-1} \log n\right\}<\frac{|W|}{2}<\ell$ by choice of $C$, at each step there is a valid choice of $w_{s}$. Since for each $x \in V\left(H^{\prime}\right)$ we have $\left|J_{x}\right| \leq 1$, this construction guarantees $(G 7 \mathrm{a})$.

We now return to following the proof of Theorem 25. We obtain $\mathcal{V}^{\prime}$ by removing the images of pre-embedded vertices, and $\mathcal{V}^{\prime \prime}$ by applying Lemma 29 . Note that here $\left(B 5^{\prime}\right)$ may be trivial, that is, the error term $C^{*} \log n$ may dominate the main term when $s$ is large, but we only require it for $s=1$ to obtain $(G 1 \mathrm{c})-(G 7 \mathrm{c})$.

Finally, we are ready to apply Lemma 38 to complete the embedding. We define $(\mathcal{J}, \mathcal{J})$ as in the proof of Theorem 25. We however let $\widetilde{W}_{i, j}$ consist of the vertices of $W_{i, j}^{\prime} \backslash X$ whose degree is at most $2 D$. By (H 6a) there are at least $\frac{1}{100 D}\left|W_{i, j}^{\prime}\right|$ of these, so that $\widetilde{\mathcal{W}}$ is a $\left(\vartheta, K_{r}^{k}\right)$-buffer, giving (DBUL 1). Now (DBUL 2) follows from $(G 2 \mathrm{c})$ and $(G 3 \mathrm{c})$. Finally, $(\mathcal{J}, \mathcal{J})$ is a $\left(\rho, \frac{1}{4} \alpha, \Delta, \Delta\right)$-restriction pair, giving (DBUL 3), exactly as in the proof of Theorem 25. However now we need to give an order $\tau^{\prime}$ on $V\left(H^{\prime}\right)$ and a set $W^{e} \subseteq V\left(H^{\prime}\right)$. The former is simply the restriction of $\tau$ to $V\left(H^{\prime}\right)$, and the set $W^{e}$ consists of all vertices $x \in V(H)$ with $\left|J_{x}\right|>0$.

We now verify the remaining conditions of Lemma 38. We claim $\left|W^{e}\right| \leq \Delta^{2}\left|V_{0}\right| \leq \varepsilon p^{\max _{x \notin W^{e}} \pi^{\tau^{\prime}}(x)} n / r_{1}$. Observe that $\pi^{\tau^{\prime}}(x) \leq \pi^{\tau}(x)+\left|J_{x}\right| \leq D+1$. For $D=1$, we have $\left|V_{0}\right| \leq C^{*} p^{-1}$, and by choice of $C$ the desired inequality follows. For $D \geq 2$, we have $\left|V_{0}\right| \leq C^{*} p^{-2}$, and again by choice of $C$ we have the desired inequality. 
The BAndwidTh TheOREM In SPARSE GRAPHS

The last condition we must verify is $(D B U L 4)$, that $\tau^{\prime}$ is a $\left(\tilde{D}, p, \varepsilon n / r_{1}\right)$-bounded order. For any vertex $x$ of $H^{\prime}$, we have $\pi^{\tau^{\prime}}(x) \leq \pi^{\tau}(x)+1 \leq D+1$, and furthermore for all vertices not in $W^{e}$ we have $\pi^{\tau^{\prime}}(x)=\pi^{\tau}(x) \leq D$. To verify $(O R D 1)$, first note that by construction the vertices of $\bigcup \widetilde{\mathcal{W}}$ have degree at most $2 D \leq \tilde{D}$. Further, observe that if $D=1$ then $H^{\prime}$ contains no triangles, and $\tilde{D}=3=D+2$. Since vertices in $N(\cup \widetilde{\mathcal{W}})$ are by construction not image restricted, so are not in $W^{e}$, this is as required for $(O R D 1)$. If on the other hand $D \geq 2$ then $\tilde{D} \geq D+3$, and again the conditions of (ORD 1) are met. Next, if $x \notin W^{e}$ then $\pi^{\tau^{\prime}}(x) \leq D$, so that (ORD 2) holds. Finally, observe that $\max _{z \notin W^{e}} \pi^{\tau^{\prime}}(z) \leq D$, and vertices $x \in N(\cup \widetilde{\mathcal{W}})$ by construction have $\pi^{\tau^{\prime}}(x)=\pi^{\tau}(x) \leq D$, so that (ORD 3 ) holds.

We can thus apply Lemma 38 to embed $H^{\prime}$ into $G^{\prime}$, completing the embedding of $H$ into $G$ as desired.

The proof of Theorem 8 from Theorem 36 follows the deduction of Theorem 7 from Theorem 25, and we omit it.

\section{The Bandwidth Theorem in bijumbled graphs}

Again, Theorem 9 is a consequence of the following.

Theorem 39. For each $\gamma>0, \Delta \geq 2$, and $k \geq 1$, there exists a constant $c>0$ such that the following holds for any $p>0$. Given $v \leq c p^{\max (4,(3 \Delta+1) / 2)} n$, suppose $\Gamma$ is $a(p, v)$-bijumbled graph, $G$ is a spanning subgraph of $\Gamma$ with $\delta(G) \geq\left(\frac{k-1}{k}+\gamma\right)$ pn, and $H$ is a $k$-colourable graph on $n$ vertices with $\Delta(H) \leq \Delta$ and bandwidth at most $\mathrm{cn}$. Suppose further that $H$ has a labelling $\mathcal{L}$ of its vertex set of bandwidth at most cn, $a(k+1)$-colouring that is $(z, c)$-zero-free with respect to $\mathcal{L}$, and where the first $\sqrt{c} n$ vertices in $\mathcal{L}$ are not given colour zero, and the first cn vertices in $\mathcal{L}$ include $c^{-1} p^{-6} v^{2} n^{-1}$ vertices in $V(H)$ that are not contained in any triangles of $H$. Then $G$ contains a copy of $H$.

The proof of Theorem 39 is a straightforward modification of that of Theorem 25. Rather than repeating the entire proof, we sketch the modifications which have to be made. Again, for more details and background on this result see [22].

Since we are working with bijumbled graphs, we need to work with regular pairs, rather than lowerregular pairs, at all times. In order to use this concept, and to work with bijumbled graphs, we need versions of Lemmas 16, 17, and 18, and Proposition 19, which work with regular pairs and with $\Gamma$ a bijumbled graph rather than a random graph. We also need the following easy proposition, which lower bounds the possible $v$ for a $(p, v)$-jumbled graph with $p>0$.

Proposition 40. Suppose $\frac{16}{n}<p<1-\frac{16}{n}$. There does not exist any $(p, v)$-bijumbled $n$-vertex graph with $v \leq \min (\sqrt{p n / 32}, \sqrt{(1-p) n / 32})$.

Proof. Suppose that $\Gamma$ is a $(p, v)$-bijumbled graph on $n$ vertices with $p \leq \frac{1}{2}$. If $\Gamma$ contains $\frac{1}{2} n$ vertices of degree at least $4 p n$, then we have $e(\Gamma) \geq p n^{2}$, and letting $A, B$ be a maximum cut of $\Gamma$, by bijumbledness we have

$$
\frac{1}{2} p n^{2} \leq e(A, B) \leq p|A||B|+v \sqrt{|A||B|} \leq \frac{1}{4} p n^{2}+\frac{1}{2} v n,
$$

and thus $v \geq p n / 2 \geq \sqrt{p n / 32}$. 
If on the contrary $\Gamma$ contains at least $\frac{1}{2} n$ vertices of degree less than $4 p n$, then let $A$ be a set of $\frac{1}{8 p}$ such vertices, and $B$ a set of $\frac{n}{4}$ vertices with no neighbours in $A$. By bijumbledness, we have

$$
0 \geq p|A||B|-v \sqrt{|A||B|}=\frac{n}{32}-v \sqrt{n /(32 p)}
$$

and thus $v \geq \sqrt{p n / 32}$. The same argument applied to $\bar{\Gamma}$ proves the $p \geq \frac{1}{2}$ case.

The following sparse blow-up lemma for jumbled graphs is proved in [2].

Lemma 41 ([2, Lemma 1.25]). For all $\Delta \geq 2, \Delta_{R^{\prime}}, \Delta_{J}, \alpha, \zeta, d>0, \kappa>1$ there exist $\varepsilon, \rho>0$ such that for all $r_{1}$ there is a $c>0$ such that if $p>0$ and

$$
\beta \leq c p^{\max (4,(3 \Delta+1) / 2)} n
$$

any $(p, \beta)$-bijumbled graph $\Gamma$ on $n$ vertices satisfies the following.

Let $R$ be a graph on $r \leq r_{1}$ vertices and let $R^{\prime} \subseteq R$ be a spanning subgraph with $\Delta\left(R^{\prime}\right) \leq \Delta_{R^{\prime}}$. Let $H$ and $G \subseteq \Gamma$ be graphs given with $\kappa$-balanced, size-compatible vertex partitions $\mathcal{X}=\left\{X_{i}\right\}_{i \in[r]}$ and $\mathcal{V}=\left\{V_{i}\right\}_{i \in[r]}$, respectively, which have parts of size at least $m \geq n /\left(\kappa r_{1}\right)$. Let $\widetilde{X}=\left\{\widetilde{X}_{i}\right\}_{i \in[r]}$ be a family of subsets of $V(H), \mathcal{J}=\left\{I_{x}\right\}_{x \in V(H)}$ be a family of image restrictions, and $\mathcal{J}=\left\{J_{x}\right\}_{x \in V(H)}$ be a family of restricting vertices. Suppose that

(JBUL 1) $\Delta(H) \leq \Delta,(H, X)$ is an R-partition, and $\widetilde{X}$ is an $\left(\alpha, R^{\prime}\right)$-buffer for $H$,

$(J B U L 2)(G, \mathcal{V})$ is an $(\varepsilon, d, p)$-regular $R$-partition, which is $(\varepsilon, d, p)$-super-regular on $R^{\prime}$, and has one-sided inheritance on $R^{\prime}$, and two-sided inheritance on $R^{\prime}$ for $\widetilde{X}$,

(JBUL 3) J and J form $a\left(\rho p^{\Delta}, \zeta, \Delta, \Delta_{J}\right)$-restriction pair.

Then there is an embedding $\psi: V(H) \rightarrow V(G)$ such that $\psi(x) \in I_{x}$ for each $x \in H$.

There are three differences between this result and Lemma 16. First, we assume a bijumbledness condition on $\Gamma$, rather than that $\Gamma$ is a typical random graph. Second, we require regular pairs in place of lower-regular pairs. Third, the number of vertices we may image restrict is much smaller. We will see that these last two restrictions do not affect our proof substantially.

Next, in [3], the following regularity inheritance lemmas for bijumbled graphs are proved.

Lemma 42 ([3, Lemma 3]). For each $\varepsilon^{\prime}, d>0$ there are $\varepsilon, c>0$ such that for all $0<p<1$ the following holds. Let $G \subseteq \Gamma$ be graphs and $X, Y, Z$ be disjoint vertex sets in $V(\Gamma)$. Assume that

- $(X, Z)$ is $\left(p, c p^{3 / 2} \sqrt{|X||Z|}\right)$-bijumbled in $\Gamma$,

- $(X, Y)$ is $\left(p, c p^{2}\left(\log _{2} \frac{1}{p}\right)^{-1 / 2} \sqrt{|X||Y|}\right)$-bijumbled in $\Gamma$, and

- $(X, Y)$ is $(\varepsilon, d, p)_{G}$-regular.

Then, for all but at most at most $\varepsilon^{\prime}|Z|$ vertices $z$ of $Z$, the pair $\left(N_{\Gamma}(z) \cap X, Y\right)$ is $\left(\varepsilon^{\prime}, d, p\right)_{G}$-regular. 
THE BANDWIDTH THEOREM IN SPARSE GRAPHS

Lemma 43 ([3, Lemma 4]). For each $\varepsilon^{\prime}, d>0$ there are $\varepsilon, c>0$ such that for all $0<p<1$ the following holds. Let $G \subseteq \Gamma$ be graphs and $X, Y, Z$ be disjoint vertex sets in $V(\Gamma)$. Assume that

- $(X, Z)$ is $\left(p, c p^{2} \sqrt{|X||Z|}\right)$-bijumbled in $\Gamma$,

- $(Y, Z)$ is $\left(p, c p^{3} \sqrt{|Y||Z|}\right)$-bijumbled in $\Gamma$,

- $(X, Y)$ is $\left(p, c p^{5 / 2}\left(\log _{2} \frac{1}{p}\right)^{-\frac{1}{2}} \sqrt{|X||Y|}\right)$-bijumbled in $\Gamma$, and

- $(X, Y)$ is $(\varepsilon, d, p)_{G}$-regular.

Then, for all but at most $\varepsilon^{\prime}|Z|$ vertices $z$ of $Z$, the pair $\left(N_{\Gamma}(z) \cap X, N_{\Gamma}(z) \cap Y\right)$ is $\left(\varepsilon^{\prime}, d, p\right)_{G^{-r e g u l a r}}$

The following two lemmas, which more closely resemble Lemmas 17 and 18, are corollaries.

Lemma 44. For each $\varepsilon_{\text {OSRIL }}, \alpha_{\text {OSRIL }}>0$ there exist $\varepsilon_{0}>0$ and $C>0$ such that for any $0<\varepsilon<\varepsilon_{0}$ and $0<p<1$, if $\Gamma$ is any $(p, v)$-bijumbled graph the following holds. For any disjoint sets $X$ and $Y$ in $V(\Gamma)$ with $|X| \geq C p^{-3} v$ and $|Y| \geq C p^{-2} v$, and any subgraph $G$ of $\Gamma[X, Y]$ which is $\left(\varepsilon, \alpha_{\text {osRIL }}, p\right)_{G \text {-regular, there }}$ are at most $C p^{-3} v^{2}|X|^{-1}$ vertices $z \in V(\Gamma)$ such that $\left(X \cap N_{\Gamma}(z), Y\right)$ is not $\left(\varepsilon_{\mathrm{OSRIL}}, \alpha_{\mathrm{OSRIL}}, p\right)_{G^{-}}$-regular.

Lemma 45. For each $\varepsilon_{\mathrm{TSRIL}}, \alpha_{\mathrm{TSRI}}>0$ there exist $\varepsilon_{0}>0$ and $C>0$ such that for any $0<\varepsilon<\varepsilon_{0}$ and $0<p<1$, if $\Gamma$ is any $(p, v)$-bijumbled graph the following holds. For any disjoint sets $X$ and $Y$ in $V(\Gamma)$ with $|X|,|Y| \geq C p^{-3} v$, and any subgraph $G$ of $\Gamma[X, Y]$ which is $\left(\varepsilon, \alpha_{\mathrm{TSRIL}}, p\right)_{G^{-}}$regular, there are at most $C p^{-6} v^{2} / \min (|X|,|Y|)$ vertices $z \in V(\Gamma)$ such that $\left(X \cap N_{\Gamma}(z), Y \cap N_{\Gamma}(z)\right)$ is not $\left(\varepsilon_{\mathrm{TSRIL}}, \alpha_{\mathrm{TSRIL}}, p\right)_{G^{-}}$ regular.

Note that the bijumbledness requirements of this lemma are such that if $Y$ and $Z$ are sets of size $\Theta(n)$, then $X$ must have size $\Omega\left(p^{-6} v^{2} n^{-1}\right)$. This is where the requirement of Theorem 39 for vertices of $H$ not in triangles comes from.

Finally, we give a bijumbled graphs version of Proposition 19. We defer its proof, which is standard, and similar to that of Proposition 19, to Appendix A.

Proposition 46. For each $\varepsilon>0$ there exists a constant $C>0$ such that for every $p>0$, any graph $\Gamma$ which is $(p, v)$-jumbled has the following property. For any disjoint $X, Y \subseteq V(\Gamma)$ with $|X|,|Y| \geq \varepsilon^{-1} p^{-1} v$, we have $e(X, Y)=(1 \pm \varepsilon) p|X||Y|$, and $e(X) \leq 2 p|X|^{2}$. Furthermore, for every $Y \subseteq V(\Gamma)$ with $|Y| \geq C p^{-1} v$, the number of vertices $v \in V(\Gamma)$ with ||$N_{\Gamma}(v, Y)|-p| Y||>\varepsilon p|Y|$ is at most $C p^{-2} v^{2}|Y|^{-1}$.

Now, using these lemmas, we can prove bijumbled graph versions of Lemmas 26 and 28, and use these to complete the proof of Theorem 39. All these proofs are straightforward modifications of those in the previous sections. Briefly, the modifications we make are to replace 'lower-regular' with 'regular' in all proofs, to replace applications of lemmas for random graphs with the bijumbled graph versions above, and to recalculate some error bounds.

The only one of our main lemmas which changes in an important way is the following Lemma for $G$.

Lemma 47 (Lemma for $G$, bijumbled graph version). For each $\gamma>0$ and integers $k \geq 2$ and $r_{0} \geq 1$ there exists $d>0$ such that for every $\varepsilon \in\left(0, \frac{1}{2 k}\right)$ there exist $r_{1} \geq 1$ and $c, C^{*}>0$ such that the following holds for any $n$-vertex $(p, v)$-bijumbled graph $\Gamma$ with $v \leq c p^{3} n$ and $p>0$. Let $G=(V, E)$ be a spanning 
subgraph of $\Gamma$ with $\delta(G) \geq\left(\frac{k-1}{k}+\gamma\right)$ pn. Then there exists an integer $r$ with $r_{0} \leq k r \leq r_{1}$, a subset $V_{0} \subseteq V$ with $\left|V_{0}\right| \leq C^{*} p^{-6} v^{2} n^{-1}$, a k-equitable vertex partition $\mathcal{V}=\left\{V_{i, j}\right\}_{i \in[r], j \in[k]}$ of $V(G) \backslash V_{0}$, and a graph $R_{r}^{k}$ on the vertex set $[r] \times[k]$ with $K_{r}^{k} \subseteq B_{r}^{k} \subseteq R_{r}^{k}$, with $\delta\left(R_{r}^{k}\right) \geq\left(\frac{k-1}{k}+\frac{\gamma}{2}\right) k r$, and such that the following are true.

(G1) $\frac{n}{4 k r} \leq\left|V_{i, j}\right| \leq \frac{4 n}{k r}$ for every $i \in[r]$ and $j \in[k]$,

(G2) $\mathcal{V}$ is $(\varepsilon, d, p)_{G}$-regular on $R_{r}^{k}$ and $(\varepsilon, d, p)_{G}$-super-regular on $K_{r}^{k}$,

(G3) both $\left(N_{\Gamma}\left(v, V_{i, j}\right), V_{i^{\prime}, j^{\prime}}\right)$ and $\left(N_{\Gamma}\left(v^{\prime}, V_{i, j}\right), N_{\Gamma}\left(v^{\prime}, V_{i^{\prime}, j^{\prime}}\right)\right)$ are $(\varepsilon, d, p)_{G^{-}}$regular pairs for every edge $\left\{(i, j),\left(i^{\prime}, j^{\prime}\right)\right\} \in E\left(R_{r}^{k}\right)$, every $v \in V \backslash\left(V_{0} \cup V_{i, j}\right)$ and $v^{\prime} \in V \backslash\left(V_{0} \cup V_{i, j} \cup V_{i^{\prime}, j^{\prime}}\right)$,

(G4) we have $(1-\varepsilon) p\left|V_{i, j}\right| \leq\left|N_{\Gamma}\left(v, V_{i, j}\right)\right| \leq(1+\varepsilon) p\left|V_{i, j}\right|$ for every $i \in[r], j \in[k]$ and every $v \in V \backslash V_{0}$.

The change here, apart from replacing 'lower-regular' with 'regular', and working in bijumbled graphs, is that $V_{0}$ may now be a much larger set. Nevertheless, the proof is basically the same.

Sketch proof of Lemma 47. We begin the proof as in that of Lemma 26, setting up the constants in the same way, with the exception that we replace Lemmas 17 and 18 with Lemmas 44 and 45, and Proposition 19 with Proposition 46. We require $C$ to be sufficiently large for Lemmas 44 and 45, and for Proposition 46. We define $C^{*}=100 k^{2} r_{1}^{3} C / \varepsilon^{*}$ as in the proof of Lemma 26, and set

$$
c=10^{-5}\left(\varepsilon^{*}\right)^{3}\left(k r_{1}\right)^{-3}\left(C^{*}\right)^{-1}
$$

We now assume $\Gamma$ is $(p, v)$-bijumbled rather than random, with $v \leq c p^{3} n$. In particular, by choice of $c$ this implies that

$$
10 k^{2} r_{1}^{2} C p^{-2} v^{2} n^{-1} \leq \varepsilon^{*} p n \quad \text { and } \quad 10 k^{2} r_{1}^{3} C p^{-6} v^{2} n^{-1} \leq \varepsilon^{*} n
$$

We obtain a regular partition, with a reduced graph containing $B_{r}^{k}$, exactly as in the proof of Lemma 26, using Proposition 46 in place of Proposition 19 to justify the use of Lemma 13. The next place where we need to change things occurs in defining $Z_{1}$, where we replace 'lower-regular' with 'regular', and in estimating the size of $Z_{1}$. Using Lemmas 44 and 45, and Proposition 19 with Proposition 46, we replace (4.1) with

$$
\left|Z_{1}\right| \leq k r_{1}^{2} C p^{-6} v^{2} n^{-1}+k r_{1}^{2} C p^{-3} v^{2} n^{-1}+2 k r_{1} C p^{-2} v^{2} n^{-1} \leq 4 k r_{1}^{2} C p^{-6} v^{2} n^{-1} \stackrel{(10.1)}{\leq} \frac{\varepsilon^{*}}{k r_{1}} n .
$$

Note that the final conclusion is as in (4.1).

We can now continue following the proof of Lemma 26 until we come to estimate the size of $Z_{2}$, where we use Proposition 46 and replace (4.2) with

$$
\left|Z_{2}\right| \leq r_{1}+k r_{1} C p^{-2} v^{2} n^{-1} \stackrel{(10.1)}{\leq} \frac{\varepsilon^{*}}{k r_{1}} p n
$$

Again, the final conclusion is as in (4.2). 
The BANDWIDTh ThEOREM IN SPARSE GRAPHS

The next change we have to make is in estimating the size of $V_{0}$, when we replace (4.6) with

$$
\left|V_{0}\right| \leq\left|Z_{1}\right|+\left|Z_{2}\right| \leq 4 k r_{1}^{2} C p^{-6} v^{2} n^{-1}+r_{1}+k r_{1} C p^{-2} v^{2} n^{-1} \leq C^{*} p^{-6} v^{2} n^{-1} .
$$

Finally, we need regular pairs in $(G 2)$ and $(G 3)$. We obtained regular pairs from Lemma 13 and in the definition of $Z_{1}$, so that we only need Proposition 12 to return regular pairs. We always apply Proposition 12 to pairs of sets of size at least $\frac{\varepsilon^{*} p n}{r_{1}}$, altering them by a factor $\varepsilon^{*}$. Now Proposition 46 shows that if $X$ and $Y$ are disjoint subsets of $\Gamma$ with $|X|,|Y| \leq\left(\varepsilon^{*} p\right)^{-1} v$, then $e_{\Gamma}(X, Y) \leq\left(1+\varepsilon^{*}\right) p|X||Y|$, as required. By choice of $c$, we have $\left(\varepsilon^{*} p\right)^{-1} v \leq\left(\varepsilon^{*}\right)^{2} p n / r_{1}$, so that the condition of Proposition 12 to return regular pairs is satisfied.

The other one of our main lemmas which requires change, Lemma 28, only requires changing 'lower-regular' to 'regular' and replacing the random graph with a bijumbled $\Gamma$. This does require some change in the proof, as we then use the bijumbled graph versions of various lemmas, whose error bounds are different.

Lemma 48 (Common neighbourhood lemma, bijumbled graph version). For each $d>0, k \geq 1$, and $\Delta \geq 2$ there exists $\alpha>0$ such that for every $\varepsilon^{*} \in(0,1)$ there exists $\varepsilon_{0}>0$ such that for every $r \geq 1$ and every $0<\varepsilon \leq \varepsilon_{0}$ there exists $c>0$ such that the following is true. For any n-vertex $\left(p, c p^{\Delta+1} n\right)$ bijumbled graph $\Gamma$ the following holds. Let $G=(V, E)$ be a (not necessarily spanning) subgraph of $\Gamma$ and $\left\{V_{i}\right\}_{i \in[k]} \cup\{W\}$ a vertex partition of a subset of $V$ such that the following are true for every $i, i^{\prime} \in[k]$.

(G1) $\frac{n}{4 k r} \leq\left|V_{i}\right| \leq \frac{4 n}{k r}$,

(G2) $\left(V_{i}, V_{i^{\prime}}\right)$ is $(\varepsilon, d, p)_{G}$-regular,

(G3) $|W| \geq \frac{\varepsilon p n}{16 k r^{2}}$, and

(G4) $\left|N_{G}\left(w, V_{i}\right)\right| \geq d p\left|V_{i}\right|$ for every $w \in W$.

Then there exists a tuple $\left(w_{1}, \ldots, w_{\Delta}\right) \in\left(\begin{array}{c}W \\ \Delta\end{array}\right)$ such that for every $\Lambda, \Lambda^{*} \subseteq[\Delta]$, and every $i \neq i^{\prime} \in[k]$ we have

$(W 1)\left|\bigcap_{j \in \Lambda} N_{G}\left(w_{j}, V_{i}\right)\right| \geq \alpha p^{|\Lambda|}\left|V_{i}\right|$,

(W2) $\left|\bigcap_{j \in \Lambda} N_{\Gamma}\left(w_{j}\right)\right| \leq\left(1+\varepsilon^{*}\right) p^{|\Lambda|} n$,

(W3) $\left(1-\varepsilon^{*}\right) p^{|\Lambda|}\left|V_{i}\right| \leq\left|\bigcap_{j \in \Lambda} N_{\Gamma}\left(w_{j}, V_{i}\right)\right| \leq\left(1+\varepsilon^{*}\right) p^{|\Lambda|}\left|V_{i}\right|$, and

(W4) $\left(\bigcap_{j \in \Lambda} N_{\Gamma}\left(w_{j}, V_{i}\right), \bigcap_{j^{*} \in \Lambda^{*}} N_{\Gamma}\left(w_{j^{*}}, V_{i^{\prime}}\right)\right)$ is $\left(\varepsilon^{*}, d, p\right)_{G^{-}}$regular if $|\Lambda|,\left|\Lambda^{*}\right|<\Delta$ and either $\Lambda \cap \Lambda^{*}=\varnothing$ or $\Delta \geq 3$ or both.

The main modifications we make to the proof of Lemma 28 are to replace Lemmas 17 and 18 with Lemmas 44 and 45, and Proposition 19 with Proposition 46, and to replace all occurrences of 'lower-regular' with 'regular'. We sketch the remaining modifications below. 
Sketch proof of Lemma 48 . We begin the proof by setting constants as in the proof of Lemma 28, but appealing to Lemmas 44 and 45, and Proposition 46, rather than their random graph equivalents.

We set $c=10^{-20} 2^{-2 \Delta} \varepsilon^{5}\left(C t_{1} k r\right)^{-4}$. Suppose $v \leq c p^{\Delta+2} n$, and that $\Gamma$ is an $n$-vertex $(p, v)$-bijumbled graph rather than a random graph.

In order to apply Lemma 31 to $G$, we need to observe that its condition is satisfied by Proposition 46 and because $\varepsilon^{-1} p^{-1} v<10^{-10} \frac{\varepsilon^{4} p n}{k^{4} r^{4}}$ by choice of $c$. The same inequality justifies further use of Proposition 46 to find the desired $W^{\prime}$. Estimating the size of $W^{\prime}$, we replace (6.1) with

$$
\left|W^{\prime}\right| \geq 10^{-11} \frac{\varepsilon^{4} p n}{t_{1} k^{4} r^{4}} \geq 10^{5} C p^{-2} v,
$$

where the final inequality is by choice of $c$.

We only need to change the statement of Claim 32 by replacing 'lower-regular' with 'regular' in $(L 1)$ and $(L 6)$. However we need to make rather more changes to its inductive proof. The base case remains trivial. In the induction step, we need to replace (6.2) with

$$
\left|\bigcap_{j \in \Lambda} N_{\Gamma}\left(w_{j}, V_{i}^{\prime}\right)\right| \geq\left(1-\varepsilon_{0}\right)^{\Delta-2} p^{\Delta-2} \frac{n}{8 t r} \geq 10^{5} C p^{-4} v
$$

where the final inequality is by choice of $c$. This, together with $\left|W^{\prime}\right| \geq 10^{5} \mathrm{Cp}^{-2} v$ from (10.2), justifies that we can apply Lemma 44. We obtain that at most $2^{\Delta} k^{2} C p^{-3} v^{2} \frac{8 k r t_{1}}{n}$ vertices $w$ in $W$ violate $(L 1)$.

The estimate on the number of vertices violating $(L 2)$ does not change.

For $(L 4)$, we need to observe that $\left|\bigcup_{j \in \Lambda} N_{\Gamma}\left(w_{j}, V_{i}^{\prime}\right)\right|=\left(1 \pm \varepsilon_{0}\right)^{|\Lambda|} p^{|\Lambda|}\left|V_{i}^{\prime}\right|$, and in particular by choice of $\varepsilon_{0}$ and $c$ this quantity is at least $C p^{-1} v$. Then Proposition 46 then gives that at most $2^{\Delta+1} k C p^{-2} v^{2} \frac{8 k r t_{1}}{n}$ vertices destroy $(L 4)$, and the same calculation gives the same bound for the number of vertices violating $(L 5)$ and $(L 3)$.

Finally, for (L6), we need to use the inequality $\left(1-\varepsilon_{0}\right)^{\Delta-1} p^{\Delta-1} \frac{n}{4 k r} \geq C p^{-2} v$, which holds by choice of $c$, to justify that Lemmas 44 and 45 can be applied as the corresponding random graph versions are in Lemma 28. We obtain quite different bounds from these lemmas, however. If $\Delta=2$, then we only use Lemma 44, with an input regular pair having both sets of size at least $\frac{n}{4 k r}$, so that the number of vertices violating (L6) in this case is at most $2^{2 \Delta} k^{2} C p^{-3} v^{2} \frac{4 k r}{n}$. If $\Delta \geq 3$, we use both Lemma 44 and 45 . The set playing the rôle of $X$ in Lemma 44 has size at least $\left(1-\varepsilon_{0}\right)^{\Delta-2} p^{\Delta-2} \frac{n}{4 k r}$, while we apply Lemma 45 with both sets of the regular pair having at least this size. As a consequence, the number of vertices violating (L6) is at most $2^{2 \Delta+1} k^{2} C p^{-6} v^{2}\left(1-\varepsilon_{0}\right)^{2-\Delta} p^{2-\Delta} \frac{4 k r}{n}$ for the case $\Delta \geq 3$.

Putting this together, for the case $\Delta=2$ we replace (6.3) with the following upper bound for the number of vertices $w \in W^{\prime}$ which cannot be chosen as $w_{\ell+1}$.

$$
2^{\Delta} k^{2} C p^{-3} v^{2} \frac{8 k r t_{1}}{n}+2^{\Delta} k \varepsilon_{\Delta}^{* *}\left|W^{\prime}\right|+3 \cdot 2^{\Delta+1} k C p^{-2} v^{2} \frac{8 k r t_{1}}{n}+2^{2 \Delta} k^{2} C p^{-3} v^{2} \frac{4 k r}{n}
$$

By choice of $c$ and $\varepsilon_{\Delta}^{* *}$, this quantity is at most $\frac{1}{2}\left|W^{\prime}\right|$, completing the induction step for $\Delta=2$. For $\Delta \geq 3$, we replace the upper bound (6.4) with

$$
2^{\Delta} k^{2} C p^{-3} v^{2} \frac{8 k r t_{1}}{n}+2^{\Delta} k \varepsilon_{\Delta}^{* *}\left|W^{\prime}\right|+3 \cdot 2^{\Delta+1} k C p^{-2} v^{2} \frac{8 k r t_{1}}{n}+2^{2 \Delta+1} k^{2} C p^{-6} v^{2}\left(1-\varepsilon_{0}\right)^{2-\Delta} p^{2-\Delta} \frac{4 k r}{n}
$$




\section{The BANDWIDTh ThEOREM IN SPARSE GRAPHS}

which by choice of $c, \varepsilon_{0}$ and $\varepsilon_{\Delta}^{* *}$ is at most $\frac{1}{2}\left|W^{\prime}\right|$, completing the induction step for $\Delta \geq 3$.

We conclude that the modified Claim 32 continues to hold, and this implies the statement of Lemma 48 as in the proof of Lemma 28.

The proof of Theorem 39 is similar to that of Theorem 25. Again, we sketch the modifications.

Sketch proof of Theorem 39. We begin as in the proof of Theorem 25, setting up constants as there, but replacing Lemma 26 with Lemma 47, Lemma 28 with Lemma 48, Lemma 16 with Lemma 41, and Proposition 19 with Proposition 46. In addition to the constants defined in the proof of Theorem 25 we require $0<c \leq 10^{-50} \varepsilon^{8} \mu \rho \xi^{2}\left(\Delta k r_{1} C\right)^{-10}$ to be small enough for Lemmas 47 and 48 .

Now, instead of assuming $\Gamma$ to be a typical random graph, suppose $v \leq c p^{\max \{4,(3 \Delta+1) / 2\}} n$, and let $\Gamma$ be an $n$-vertex $(p, v)$-bijumbled graph. By Proposition 40 we have

$$
p \geq C^{*}\left(\frac{\log n}{n}\right)^{1 / 2} .
$$

We continue following the proof of Theorem 25 . We now assume the first $\beta n$ vertices of $\mathcal{L}$ include $C p^{-6} v^{2} n^{-1}$ vertices that are not contained in any triangles of $H$. We appeal to Lemma 47 rather than Lemma 26 to obtain a partition of $V(G)$. This partition has $\left|V_{0}\right| \leq C^{*} p^{-6} v^{2} n^{-1}$ (which is different to the upper bound in the proof of Theorem 25), but still satisfies ( $G 1 \mathrm{a})$ and $(G 4 \mathrm{a})$, and $(G 2 \mathrm{a})$ and $(G 3 \mathrm{a})$ when 'lower-regular' is replaced by 'regular' in both statements.

The application of Lemma 27 is identical. The application of Lemma 22 is also identical, and the deduction of (8.1) is still valid by (10.3). The pre-embedding is also identical, except that we replace each occurrence of $C^{*} \max \left\{p^{-2}, p^{-1} \log n\right\}$ with $C^{*} p^{-6} v^{2} n^{-1}$, and that we replace the application of Proposition 19 justifying that at each visit to Line 1 we have at least $\frac{1}{4} \mu$ pn choices with an application of Proposition 46. To verify the condition of the latter, and to see that this yields a contradiction we use the inequality $|Z| \geq \frac{1}{100(\Delta+1)} \mu p n \geq 2 C^{*} p^{-2} v^{2} \frac{8 r}{\varepsilon n}$, which holds by choice of $c$.

Moving on, we justify Claim 35 by observing that $\frac{\varepsilon n}{4 k r_{1}} \geq C p^{-1} v$, which allows us to apply Proposition 46 in place of Proposition 19, and that $2 k r C^{*} p^{-2} v^{2} \frac{4 k r_{1}}{\varepsilon n} \leq \frac{|Y|}{2}$, both inequalities following by choice of $c$.

Now Lemma 48, in place of Lemma 28, finds $w_{1}, \ldots, w_{\ell}$. Our construction of $f^{*}$, and its properties, is identical, while Lemma 48 gives $(G 1 \mathrm{a})-(G 8 \mathrm{a})$, with 'lower-regular' replaced by 'regular' in $(G 2 \mathrm{a}),(G 3 \mathrm{a})$ and $(G 7 \mathrm{a})$. The deduction of $(G 1 \mathrm{~b})-(G 8 \mathrm{~b})$ is identical, except that we use the 'regular' consequence of Proposition 12. To justify this, observe that each time we apply Proposition 12, we apply it to a regular pair with sets of size at least $\left(1-\varepsilon^{*}\right) p^{\Delta-1} \frac{n}{4 k r}$ by $(G 1 \mathrm{a})$ and $(G 6 \mathrm{a})$, and we change the set sizes by a factor $(1 \pm 2 \mu)$, so that Proposition 46 gives the required condition. To check this in turn, we need to observe that $2 \mu\left(1-\varepsilon^{*}\right) p^{\Delta-1} \frac{n}{4 k r} \geq 100 \mu^{-1} p^{-1} v$, which follows by choice of $c$. We can thus replace 'lower-regular' with 'regular' in $(G 2 \mathrm{~b}),(G 3 \mathrm{~b})$ and $(G 7 \mathrm{~b})$.

Next, we still have $3 \Delta^{r+10}\left|V_{0}\right| \leq \frac{1}{10} \xi_{n}$, so that $\left|V_{i, j}^{\prime}\right|=\left|W_{i, j}^{\prime}\right| \pm \xi_{n}$ is still valid for each $i \in[r]$ and $j \in[k]$. This, together with (10.3), Proposition 46, and the inequality $\frac{1}{50000 k r_{1}} \varepsilon^{2} \xi p n \leq 100 \varepsilon^{-2} \xi^{-1} p^{-1} v$, justifies that we can apply Lemma 29 to obtain $(G 1 \mathrm{c})-(G 6 \mathrm{c})$, with 'lower-regular' replaced by 'regular' in $(G 2 \mathrm{c})$ and $(G 3 \mathrm{c})$. Finally, to obtain $(G 7 \mathrm{c})$ with 'lower-regular' replaced by 'regular', we use Proposition 12 , with the condition to output regular pairs guaranteed by the inequality $10^{-20} \varepsilon^{4} k^{-3} r_{1}^{-3} p^{\Delta-1} n \geq$ $10^{20} \varepsilon^{-4} k^{3} r_{1}^{3} C p^{-1} v$, which follows by choice of $c$, and Proposition 46. 
Finally, we verify the conditions for Lemma 41. The only point where we have to be careful is with the number of image restricted vertices. The total number of image restricted vertices in $H^{\prime}$ is at most $\Delta^{2}\left|V_{0}\right| \leq \Delta^{2} C^{*} p^{-6} v^{2} n^{-1}$, which by choice of $c$ and by $(G 1 \mathrm{c})$ is smaller than $\rho p^{\Delta}\left|V_{i, j}\right|$ for any $i \in[r]$ and $j \in[k]$, justifying that $(\mathcal{J}, \mathcal{J})$ is indeed a $\left(\rho p^{\Delta}, \frac{1}{4} \alpha, \Delta, \Delta\right)$-restriction pair. The remaining conditions of Lemma 41 are verified as in the proof of Theorem 25, and applying it we obtain an embedding $\phi$ of $H^{\prime}$ into $G \backslash \operatorname{im}\left(\phi_{t^{*}}\right)$, so tha $\phi \cup \phi_{t^{*}}$ is the desired embedding of $H$ into $G$.

Finally, the deduction of Theorem 9 from Theorem 39 is essentially the same as that of Theorem 7 from Theorem 25, and we omit it.

\section{Concluding remarks}

\subsection{General spanning subgraphs}

Our main theorems place restrictions on the graphs $H$ with respect to whose containment random or pseudorandom graphs have local resilience. As was shown by Huang, Lee and Sudakov [24], such restrictions are necessary. Given $\varepsilon>0$, if $\Gamma$ is either a typical random graph $G(n, p)$ or a pseudorandom graph with density $p$, and $p$ is sufficiently small, then one can delete edges from $\Gamma$ in order to remove all triangles at a given vertex $v$, without deleting more than $\varepsilon p n$ edges at any vertex. Thus if $H$ is any graph all of whose vertices are in triangles, if $p=o(1)$ the local resilience of $\Gamma$ with respect to containment of $H$ is $o(1)$.

This leads to the question: if we instead restrict $G$, requiring in addition to the conditions of Theorem 7 that $G$ contains a positive proportion of the copies of $K_{\Delta+1}$ in $\Gamma$ at each vertex, is it true that $G$ will contain any $k$-colourable, bounded degree spanning subgraph $H$ with sublinear bandwidth without further restriction? We study this question in a forthcoming companion note to this paper, together with Schnitzer [1].

\subsection{Optimality of Theorem 7}

Recall that Huang, Lee and Sudakov [24] proved that the restriction on $H$ that $C^{*} p^{-2}$ vertices should not be in triangles is necessary for all $p$. For $p$ constant, they proved a version of Theorem 7 , but the number of vertices in $H$ they require to have independent neighbourhood grows as a tower type function of $p^{-1}$, and they also require these vertices to be well-distributed in the bandwidth order, so that our result is strictly stronger than theirs.

On the other hand, we do not believe that the lower bound on $p$ in Theorem 7 is optimal. For $\Delta=2$, the statement is certainly false for $p \ll n^{-1 / 2}$, since then $G(n, p)$ has a.a.s. local resilience $o(1)$ with respect to containing even one triangle. It seems likely that the statement is true down to this point, a log factor improvement on our result. For $\Delta=3$, the statement as written is false for $p \ll n^{-1 / 3}$. Briefly, the reason for this is that in expectation a vertex is in $O\left(p^{6} n^{3}\right)$ copies of $K_{4}$ in $G(n, p)$, and (with some work) this implies that there is a.a.s. a subgraph of $G(n, p)$ with minimum degree very close to $p n$ and $p^{-5} n^{-1}$ vertices not in copies of $K_{4}$. For $p \ll n^{-1 / 3}, p^{-5} n^{-1} \gg p^{-2}$, so that we would also have to insist on many vertices of $H$ not being in copies of $K_{4}$ to accommodate this. Generalising this, we obtain the following conjecture. 
Conjecture 49. For each $\gamma>0, \Delta \geq 2$, and $k \geq 1$, there exist constants $\beta^{*}>0$ and $C^{*}>0$ such that the following holds asymptotically almost surely for $\Gamma=G(n, p)$ if $p \geq C^{*} n^{-2 /(\Delta+2)}$. Let $G$ be a spanning subgraph of $\Gamma$ with $\delta(G) \geq\left(\frac{k-1}{k}+\gamma\right)$ pn and let $H$ be a $k$-colourable graph on $n$ vertices with $\Delta(H) \leq \Delta$, bandwidth at most $\beta^{*} n$, there are at least $C^{*} p^{-2}$ vertices in $V(H)$ that are not contained in any triangles of $H$, and at least $C^{*} p^{-(\Delta+2)(\Delta-1) / 2} n^{2-\Delta}$ vertices in $V(H)$ which are not in $K_{\Delta+1}$. Then $G$ contains a copy of $H$.

This conjecture seems to be hopelessly out of reach with our current state of knowledge. We cannot even prove that $G(n, p)$ itself is universal for graphs on $\frac{n}{2}$ vertices with maximum degree $\Delta$. The best current result in this direction is due to Conlon, Ferber, Nenadov and Škorić [17], who show that for $\Delta \geq 3$, if $p \gg n^{-1 /(\Delta-1)} \log ^{5} n$ then $G(n, p)$ is a.a.s. universal for graphs on $(1-o(1)) n$ vertices of maximum degree $\Delta$, finally breaking the $n^{-1 / \Delta}$ barrier which is reached by several papers, but still far from the conjectured truth. It is possible that their methods could be used to prove a version of Theorem 7 for almost-spanning $H$ in sparser random graphs, but this does not appear to be straightforward.

\subsection{Optimality of Theorem 8}

The 'extra' restriction we place in Theorem 8, of having many vertices of $H$ which are neither in triangles nor four-cycles, is an artifact of our proof. It would be possible to remove the stipulation regarding four-cycles - one can prove a version of Lemma 28 capable of embedding vertices in a degeneracy order. However this comes at the cost of a worse lower bound on $p$. It seems likely that one would be able to obtain a result for $p \gg\left(\frac{\log n}{n}\right)^{1 /(2 D+2)}$, but we did not check the details.

As with Theorem 7, we expect that the bound $p \geq\left(\frac{\log n}{n}\right)^{1 /(2 D+1)}$ in Theorem 8 is far from the truth: again the exponent is most likely a factor of roughly 2 too small. Again, however, proving such a statement in general seems hopeless. Nevertheless, in one interesting case we can substantially improve on Theorem 8. Specifically, if $H$ is an $F$-factor for some fixed $F$, then we can follow the proof of Theorem 8 , but set $\tilde{D}=D+3$. We can do this because we choose a degeneracy order on $H$ in which the copies of $F$ are segments. We obtain a version of Theorem 8 in which $H$ is required to be an $F$-factor, where $F$ is $D$-degenerate, but the lower bound on $p$ improves to $p \geq C^{*}\left(\frac{\log n}{n}\right)^{1 /(D+3)}$. This is still not optimal, but at least the exponent is asymptotically optimal as $D$ grows, rather than being off by a factor of two in the limit. For some specific $F$ one can improve this bound further; moreover for $F$-factors one can slightly improve on Lemma 38 (see the concluding remarks of [2]).

\subsection{Optimality of Theorem 9}

The requirement of $C^{*} p^{-6} v^{2} n^{-1}$ vertices of $H$ not in triangles comes from Lemma 43. This lemma is proved in [3], where it is conjectured that the bijumbledness requirement is not optimal. What exactly the optimal result should be is not clear. When $|X|=|Y|=|Z|=\frac{n}{3}$, a construction of Alon [4] shows that $\left(p, c p^{2} n\right)$-bijumbledness is necessary for some $c>0$, but in our application we are interested in $Y$ and $Z$ being of order $n$, and $X$ much smaller.

We also do not believe that the bijumbledness requirement of Theorem 9 is optimal. This requirement comes from Lemma 41, and it is suggested there that the statement could still hold given only $\left(p, c p^{\Delta+C}\right)$ bijumbledness for some $C$. Such an improvement there would immediately improve the results here 
correspondingly. It is generally conjectured that substantial further improvement is not possible, in the strong form that it is likely that for some $C>0$ and all $\Delta$ there exists $c>0$ such that for all large $n$ an $n$-vertex $\left(p, c p^{\Delta-C}\right)$-bijumbled graph exists which does not contain $K_{\Delta+1}$ at all.

\section{Appendix}

\section{A Tools}

We collect in this appendix proofs of results which are more or less standard but which we could not find in the form we require in the literature. We begin by showing that small alterations to regular pairs give us regular pairs.

Proof of Proposition 12. Let $A \subseteq \hat{X}$ and $B \subseteq \hat{Y}$ such that $|A| \geq \hat{\varepsilon}|\hat{X}|$ and $|B| \geq \hat{\varepsilon}|\hat{Y}|$ be given. Define $A^{\prime}:=A \cap X$ and $B^{\prime}:=B \cap Y$ and note that

$$
\left|A^{\prime}\right| \geq|A|-\mu|X| \geq \hat{\varepsilon}|\hat{X}|-\mu|X| \geq \hat{\varepsilon}(1-\mu)|X|-\mu|X| \geq(\hat{\varepsilon}-2 \sqrt{\mu})|X| \geq \varepsilon|X|
$$

by the definition of $\hat{\varepsilon}$. Analogously, one can show that $\left|B^{\prime}\right| \geq \varepsilon|Y|$. Since $(X, Y)$ is an $(\varepsilon, d, p)$-regular pair, we know that $d_{p}\left(A^{\prime}, B^{\prime}\right) \geq d-\varepsilon$. Furthermore, we have

$$
\left|A^{\prime}\right| \geq|A|-\mu|X| \geq|A|-\mu \frac{|A|}{\hat{\varepsilon}} \geq(1-\sqrt{\mu})|A|
$$

and by an analogous calculation we get $\left|B^{\prime}\right| \geq(1-\sqrt{v})|B|$. For the number of edges between $A$ and $B$ we get

$$
\begin{aligned}
e(A, B) & \geq e\left(A^{\prime}, B^{\prime}\right) \geq(d-\varepsilon) p\left|A^{\prime}\right|\left|B^{\prime}\right| \geq(d-\varepsilon) p(1-\sqrt{\mu})(1-\sqrt{v})|A||B| \\
& \geq(d-\varepsilon-2 \sqrt{\mu}-2 \sqrt{v}) p|A||B| \geq(d-\hat{\varepsilon}) p|A||B| .
\end{aligned}
$$

Therefore we have

$$
d_{p}(A, B) \geq d-\hat{\varepsilon},
$$

which finishes the proof.

Now suppose that $(X, Y)$ is $(\varepsilon, d, p)$-fully-regular. Let $d^{\prime}$ be such that $d_{p}\left(A^{\prime}, B^{\prime}\right)=d^{\prime} \pm \varepsilon$ for any $A^{\prime} \subseteq X$ and $B^{\prime} \subseteq Y$ with $\left|A^{\prime}\right| \geq \varepsilon|X|$ and $\left|B^{\prime}\right| \geq \varepsilon|Y|$. Let $A \subseteq \hat{X}$ and $B \subseteq \hat{Y}$ with $|A| \geq \hat{\varepsilon}|\hat{X}|$ and $|B| \geq \hat{\varepsilon}|\hat{Y}|$ be given. As above, we obtain $e(A, B) \geq\left(d^{\prime}-\hat{\varepsilon}\right) p|A||B|$. We also have

$$
\begin{aligned}
e(A, B) & \leq e\left(A^{\prime}, B^{\prime}\right)+e\left(A^{\prime}, B \backslash B^{\prime}\right)+e\left(A \backslash A^{\prime}, B\right) \\
& \leq\left(d^{\prime}+\varepsilon\right) p\left|A^{\prime}\right|\left|B^{\prime}\right|+(1+\mu+v) p\left|A^{\prime}\right| v|B|+(1+\mu+v) p \mu|A||B| \\
& \leq\left(d^{\prime}+\hat{\varepsilon}\right)|A||B|,
\end{aligned}
$$

so that $(\hat{X}, \hat{Y})$ is $(\varepsilon, d, p)$-fully-regular, as desired.

Next, we prove the Sparse Regularity Lemma variant Lemma 31, whose proof follows [40]. 
The BANDWIDTh ThEOREM IN SPARSE GRAPHS

Proof of Lemma 31. Given $\varepsilon>0$ and $s$, let $L=100 s^{2} \varepsilon^{-1}$. Let $n_{1}=1$, and for each $j \geq 2$ let $n_{j}=$ $10000 \varepsilon^{-1} n_{j} 2^{s n_{j}}$. Let $t_{1}=n_{1000 \varepsilon^{-5}\left(L^{2}+16 L s^{2}\right)+1}$.

We define the energy of a pair of disjoint sets $P, P^{\prime}$ contained in respectively $V_{i}$ and $V_{i^{\prime}}$ to be

$$
\mathcal{E}\left(P, P^{\prime}\right):=\frac{|P|\left|P^{\prime}\right| \min \left(d_{p}\left(P, P^{\prime}\right)^{2}, 2 L d_{p}\left(P . P^{\prime}\right)-L^{2}\right)}{\left|V_{i}\right|\left|V_{i^{\prime}}\right|} .
$$

Note that this quantity is convex in $d_{p}\left(P, P^{\prime}\right)$. Now given a partition $\mathcal{P}$ refining $\left\{V_{i}\right\}_{i \in[s]}$, we define the energy of $\mathcal{P}$ to be

$$
\mathcal{E}(\mathcal{P}):=\sum_{\left\{P, P^{\prime}\right\} \subseteq \mathcal{P}} \mathcal{E}\left(P, P^{\prime}\right) .
$$

We now construct a succession of partitions $\mathcal{P}_{j+1}$ for each $j \geq 1$, refining $\mathcal{P}_{1}:=\left\{V_{i}\right\}_{i \in[s]}$. We claim that for each $j$, the following hold.

$(R 1) \mathcal{P}_{j}$ partitions $V_{i}$ into between $n_{j}$ and $\left(1+\frac{1}{100} \varepsilon\right) n_{j}$ sets, of which the largest $n_{j}$ are equally sized.

(R2) $\mathcal{E}(\mathcal{P}) \geq \frac{1}{1000} \varepsilon^{5} j$.

We stop if $\mathcal{P}_{j}$ is $\left(\frac{1}{2} \varepsilon, p\right)$-regular. If not, we apply the following procedure.

For each pair of $\mathcal{P}_{j}$ which is not $\left(\frac{1}{2} \varepsilon, 0, p\right)$-regular, we take a witness of its irregularity, consisting of a subset of each side of the pair. We let $\mathcal{P}_{j}^{\prime}$ be the union of the Venn diagrams of all witness sets in each part of $\mathcal{P}_{j}$. Since $\mathcal{P}_{j}$ is not $\left(\frac{1}{2} \varepsilon, p\right)$-regular, there are at least $\frac{1}{2} \varepsilon s^{2} n_{j}^{2}$ pairs which are not $\left(\frac{1}{2} \varepsilon, 0, p\right)$-regular. By choice of $L$ and by $(R 1)$, at least $\frac{1}{4} \varepsilon s^{2} n_{j}^{2}$ of these pairs have density not more than $\frac{1}{2} L$. By the defect Cauchy-Schwarz inequality, just from refining these pairs we conclude that $\mathcal{E}\left(\mathcal{P}_{j}^{\prime}\right) \geq \mathcal{E}\left(\mathcal{P}_{j}\right)+\frac{1}{1000} \varepsilon^{5}$. Note that, by convexity of $\mathcal{E}\left(P, P^{\prime}\right)$ in $d_{p}(P, P)$, refining the other pairs does not affect $\mathcal{E}\left(P_{j}^{\prime}\right)$ negatively.

We now let $\mathcal{P}_{j+1}$ be obtained by splitting each set of $\mathcal{P}^{\prime}{ }_{j}$ within each $V_{i}$ into sets of size $\frac{1000-\varepsilon}{1000 n_{j+1}}\left|V_{i}\right|$ plus at most one smaller set. Again by Jensen's inequality, we have $\mathcal{E}\left(\mathcal{P}_{j+1}\right) \geq \mathcal{E}\left(\mathcal{P}_{j}^{\prime}\right)$, giving $(R 2)$. Since $\mathcal{P}_{j}^{\prime}$ partitions each $V_{i}$ into at most $n_{j} 2^{s n_{j}}=\frac{1}{10000} \varepsilon n_{j+1}$, the total number of smaller sets is at most $\frac{1}{10000} \varepsilon n_{j+1}$. This gives $(R 1)$.

Now observe that for any partition $\mathcal{P}$ refining $\mathcal{P}_{1}$, we have $\mathcal{E}(\mathcal{P}) \leq L^{2}+16 L s^{2}$. It follows that this procedure must terminate with $j \leq 1000 \varepsilon^{-5}\left(L^{2}+16 L s^{2}\right)+1$. The final $\mathcal{P}_{j}$ is thus $\left(\frac{1}{2} \varepsilon, p\right)$-regular. For each $i \in[s]$, let $V_{i, 0}$ consist of the union of all but the largest $n_{j}$ parts of $\mathcal{P}_{j}$. Let $\mathcal{P}$ be the partition of $\bigcup_{i \in[s]} V_{i} \backslash V_{i, 0}$ given by $\mathcal{P}_{j}$. This is the desired equitable $(\varepsilon, p)$-regular refinement of $\left\{V_{i} \backslash V_{i, 0}\right\}_{i \in[s]}$.

Using Lemma 31 (purely in the interests of self-containment, as we could also use the results of [28]), we now prove Lemma 13.

Proof of Lemma 13. Given $\varepsilon>0$ and $r_{0}$, without loss of generality we assume $\varepsilon \leq \frac{1}{10}$. Let $t_{1}$ be returned by Lemma 31 for input $\frac{1}{1000} \varepsilon^{2} s^{-1}$ and $s=100 r_{0} \varepsilon^{-1}$. Let $r_{1}=s t_{1}$.

Given $\alpha>0$, let $G$ be an $n$-vertex graph with minimum degree $\alpha p n$. Let $\left\{V_{i}\right\}_{i \in[s]}$ be an arbitrary partition of $V(G)$ into sets of as equal as possible size. By assumption, we have $e\left(V_{i}, V_{i^{\prime}}\right) \leq 2 p\left|V_{i}\right|\left|V_{i^{\prime}}\right|$ for each $i \neq i^{\prime}$. Furthermore, if $V_{i}$ is a part with $e\left(V_{i}\right) \geq 3 p\left|V_{i}\right|^{2}$, then taking a maximum cut $A, A^{\prime}$ of $V_{i}$ we have $e\left(A, A^{\prime}\right) \geq \frac{3}{2} p\left|V_{i}\right|^{2}$. Enlarging the smaller of $A$ and $A^{\prime}$ if necessary, we have a pair of sets both of size at most $\left|V_{i}\right|$ between which there are at least $\frac{3}{2} p\left|V_{i}\right|^{2}$ edges, again contradicting the assumption 
of Lemma 13. Thus $G$ satisfies the conditions of Lemma 31 with input $\frac{1}{1000} \varepsilon^{2} s^{-1}$ and $s$. Applying that lemma, we obtain a collection $\left\{V_{i, 0}\right\}_{i \in[s]}$ of sets, and an $(\varepsilon, p)$-regular partition $\mathcal{P}$ of $\bigcup_{i \in[s]} V_{i} \backslash V_{i, 0}$ which partitions each $V_{i} \backslash V_{0}$ into $t \leq t_{1}$ sets. Note that $s \leq|\mathcal{P}| \leq r_{1}$ by construction.

Now let $V_{0}^{\prime}$ be the union of the $V_{i, 0}$ for $i \in[s]$, any sets $W \in \mathcal{P}$ that lie in more than $\frac{1}{4} \varepsilon s t$ pairs which are $\operatorname{not}\left(\frac{1}{1000} \varepsilon, p\right)$-regular, and at most two vertices from each set $W \in \mathcal{P}$ in order that the partition of $V(G) \backslash V_{0}^{\prime}$ induced by $\mathcal{P}$ is an equipartition. Because the total number of pairs which are not $\left(\frac{1}{1000} \varepsilon, p\right)$-regular is at most $\frac{1}{1000} \varepsilon^{2} s^{-1}\left(r_{0} t\right)^{2}$, the number of such sets in any given $V_{i}$ is at most $\frac{1}{100} \varepsilon t$, so $\left|V_{i, 0}^{\prime}\right|$ has size at most $\frac{1}{50} \varepsilon\left|V_{i}\right|$, and the number of parts of $\mathcal{P}$ in $V_{i} \backslash V_{i, 0}^{\prime}$ is larger than $\frac{t}{2}$. Thus the partition $\mathcal{P}^{\prime}$ of $V(G) \backslash V_{0}^{\prime}$ induced by $\mathcal{P}$ is an $(\varepsilon, p)$-regular equipartition of $V(G) \backslash V_{0}^{\prime}$, and we have $\left|V_{0}^{\prime}\right| \leq \varepsilon n$.

We claim that this partition $\mathcal{P}^{\prime}$ has all the properties we require. It remains only to check that for each $d \in[0,1]$, the $d$-reduced graph of $\mathcal{P}^{\prime}$ has minimum degree at least $(\alpha-d-\varepsilon) t^{\prime}$. Suppose that $P$ is a part of $\mathcal{P}^{\prime}$. Now we have $e(P) \leq 3 p|P|^{2}$, since otherwise, as before, a maximum cut $A, A^{\prime}$ of $P$ has at least $\frac{3}{2} p|P|^{2}<\frac{1}{20} \varepsilon p|P| n$ edges, yielding a contradiction to the assumption on the maximum density of pairs of $G$. By construction, $P$ lies in at most $\frac{1}{2} \varepsilon t^{\prime}$ pairs which are not $(\varepsilon, p)$-regular, and these contain at most $\left(1+\frac{1}{10} \varepsilon\right) p|P|\left(\frac{1}{2} \varepsilon t^{\prime}|P|\right)<\frac{3}{4} \varepsilon p|P| n$ edges of $G$. We conclude that at least $\alpha p|P| n-\frac{7}{8} \varepsilon p|P| n$ edges of $G$ leaving $P$ lie in $(\varepsilon, p)$-regular pairs of $\mathcal{P}^{\prime}$. Of these, at most $d p|P| n$ can lie in pairs of density less than $p$, so that the remaining at least $\left(\alpha-d-\frac{7}{8} \varepsilon\right) p|P| n$ edges lie in $(\varepsilon, d, p)$-regular pairs. If so many edges were in less than $(\alpha-d-\varepsilon) t^{\prime}$ pairs leaving $P$, this would contradict our assumption on the maximum density of $G$, so that we conclude $P$ lies in at least $(\alpha-d-\varepsilon) t^{\prime}$ pairs which are $(\varepsilon, d, p)$-regular, as desired.

Proof of Proposition 46. Given $\varepsilon>0$, set $C^{\prime}=100 \varepsilon^{-2}$ and $C=200 C^{\prime} \varepsilon^{-1}$. Suppose that $\Gamma$ is $(p, v)$ bijumbled.

First, given disjoint $X, Y \subseteq V(\Gamma)$ with $|X|,|Y| \geq \varepsilon^{-1} p^{-1} v,(p, v)$-bijumbledness of $\Gamma$ we have $e(X, Y)=p|X||| Y \mid \pm v \sqrt{|X||Y|}$, and we need only verify that $v \sqrt{|X||Y|} \leq \varepsilon p|X||Y|$, which follows from the lower bound on $|X|,|Y|$.

For the second property, let $(A, B)$ be a maximum cut of $X$. We have $e(A, B) \geq \frac{1}{2} e(X)$, and $|A||B| \leq$ $\frac{1}{4}|X|^{2}$. By $(p, v)$-bijumbledness of $\Gamma$, we conclude

$$
e(X) \leq 2 e(A, B) \leq 2 p|A||B|+2 v \sqrt{|A||B|} \leq \frac{1}{2} p|X|^{2}+v|X|
$$

so that it is enough to verify $v|X| \leq p|X|^{2}$, which duly follows from the lower bound on $|X|$.

Now let $Y \subseteq V(\Gamma)$ have size at least $C p^{-1} v$. We first show that there are at most $C^{\prime} p^{-2} v^{2}|Y|^{-1}$ vertices in $\Gamma$ which have less than $(1-\varepsilon) p|Y|$ neighbours in $Y$. If this were false, then we could choose a set $X$ of $C^{\prime} p^{-2} v^{2}|Y|^{-1}$ vertices in $\Gamma$ which have less than $(1-\varepsilon) p|Y|$ neighbours in $Y$. Since by choice of $C$ we have $(1-\varepsilon) p|Y| \leq\left(1-\frac{\varepsilon}{2}\right) p|Y \backslash X|$, we see that $e(X, Y \backslash X)<\left(1-\frac{\varepsilon}{2}\right) p|X||Y \backslash X|$. Since

$$
v \sqrt{|X||Y|}=v \sqrt{C^{\prime} p^{-2} v^{2}}=\sqrt{C^{\prime}} v^{2} p^{-1}<\frac{\varepsilon}{2} p|X||Y \backslash X|
$$

this is a contradiction to $(p, v)$-bijumbleness of $\Gamma$.

Next we show that there are at most $2 C^{\prime} p^{-2} v^{2}|Y|^{-1}$ vertices of $\Gamma$ which have more than $(1+\varepsilon) p|Y|$ neighbours in $Y$. Again, if this is not the case we can let $X$ be a set of $2 C^{\prime} p^{-2} v^{2}|Y|^{-1}$ vertices of $\Gamma$ with more than $(1+\varepsilon) p|Y|$ neighbours in $Y$. 
If there are more than $\frac{1}{2}|X|$ vertices of $X$ with more than $\frac{1}{2} \varepsilon p|Y|$ neighbours in $X$, then we have $e(X) \geq \frac{1}{8} \varepsilon p|X||Y|$. Taking a maximum cut $A, B$ of $X$, we have $e(A, B) \geq \frac{1}{16} \varepsilon p|X||Y|$, and by $(p, v)$ bijumbledness of $\Gamma$ we therefore have

$$
\frac{1}{16} \varepsilon p|X||Y| \leq p|A||B|+v \sqrt{|A||B|} \leq \frac{1}{4} p|X|^{2}+\frac{1}{2} v|X|,
$$

and since $|X| \leq \frac{1}{100} \varepsilon|Y|$, we conclude $|Y| \leq 100 \varepsilon^{-1} p^{-1} v$, a contradiction to the choice of $C$.

We conclude that there are $\frac{1}{2}|X|$ vertices $X^{\prime}$ of $X$ have at most $\frac{1}{2} \varepsilon p|Y|$ neighbours in $X$, and hence at least $\left(1+\frac{1}{2} \varepsilon\right) p|Y|$ neighbours in $Y \backslash X$. By $(p, v)$-bijumbledness of $\Gamma$ we have

$$
\frac{1}{2}|X|\left(1+\frac{1}{2} \varepsilon\right) p|Y| \leq e\left(X^{\prime}, Y \backslash X\right) \leq \frac{1}{2} p|X||Y|+v \sqrt{\frac{1}{2} p|X||Y|},
$$

from which we have $\varepsilon C^{\prime} p^{-1} v^{2} \leq 2 \sqrt{C^{\prime}} v^{2} p^{-1}$, a contradiction to the choice of $C^{\prime}$.

\section{Acknowledgements}

We thank two anonymous referees for valuable comments that improved our exposition.

\section{References}

[1] Peter Allen, Julia Böttcher, Julia Ehrenmüller, Jakob Schnitzer, and Anusch Taraz, A spanning bandwidth theorem in random graphs, Submitted, arXiv:1911.03958. 52

[2] Peter Allen, Julia Böttcher, Hiêp Hàn, Yoshiharu Kohayakawa, and Yury Person, Blow-up lemmas for sparse graphs, Submitted, arXiv:1612.00622. 3, 4, 5, 6, 8, 10, 43, 46, 53

[3] Peter Allen, Julia Böttcher, Jozef Skokan, and Maya Stein, Regularity inheritance in pseudorandom graphs, Random Structures Algorithms 56 (2020), no. 2, 306-338. 6, 46, 47, 53

[4] Noga Alon, Explicit Ramsey graphs and orthonormal labelings, Electron. J. Combin. 1 (1994), R12. 53

[5] Noga Alon, Michael Capalbo, Yoshiharu Kohayakawa, Vojtěch Rödl, Andrzej Ruciński, and Endre Szemerédi, Universality and tolerance (extended abstract), 41st Annual Symposium on Foundations of Computer Science (Redondo Beach, CA, 2000), IEEE Comput. Soc. Press, Los Alamitos, CA, 2000, pp. 14-21. 4

[6] József Balogh, Béla Csaba, and Wojciech Samotij, Local resilience of almost spanning trees in random graphs, Random Structures Algorithms 38 (2011), no. 1-2, 121-139. 4, 5

[7] József Balogh, Choongbum Lee, and Wojciech Samotij, Corrádi and Hajnal's theorem for sparse random graphs, Combin. Probab. Comput. 21 (2012), no. 1-2, 23-55. 4, 6 
Peter Allen, Julia Böttcher, Julia Ehrenmüller, Anusch Taraz

[8] Béla Bollobás, Modern graph theory, Graduate Texts in Mathematics, vol. 184, Springer-Verlag, New York, 1998. 7

[9] Julia Böttcher, Peter Heinig, and Anusch Taraz, Embedding into bipartite graphs, SIAM J. Discrete Math. 24 (2010), no. 4, 1215-1233. 2

[10] Julia Böttcher, Yoshiharu Kohayakawa, and Anusch Taraz, Almost spanning subgraphs of random graphs after adversarial edge removal, Combin. Probab. Comput. 22 (2013), no. 5, 639-683. 4, 6, 8,18

[11] Julia Böttcher, Klaas P. Pruessmann, Anusch Taraz, and Andreas Würfl, Bandwidth, expansion, treewidth, separators and universality for bounded-degree graphs, European J. Combin. 31 (2010), no. $5,1217-1227.2$

[12] Julia Böttcher, Mathias Schacht, and Anusch Taraz, Proof of the bandwidth conjecture of Bollobás and Komlós, Math. Ann. 343 (2009), no. 1, 175-205. 1, 2, 6, 18

[13] Julia Böttcher, Anusch Taraz, and Andreas Würfl, Spanning embeddings of arrangeable graphs with sublinear bandwidth, Random Structures Algorithms 48 (2016), no. 2, 270-289. 2, 16, 23

[14] D. Conlon and W. T. Gowers, Combinatorial theorems in sparse random sets, Ann. of Math. (2) 184 (2016), no. 2, 367-454. 2

[15] D. Conlon, W. T. Gowers, W. Samotij, and M. Schacht, On the KŁR conjecture in random graphs, Israel J. Math. 203 (2014), no. 1, 535-580. 4

[16] D. Conlon and R. Nenadov, Size Ramsey numbers of triangle-free graphs with bounded degree, Manuscript. 3

[17] David Conlon, Asaf Ferber, Rajko Nenadov, and Nemanja Škorić, Almost-spanning universality in random graphs, Random Structures Algorithms 50 (2017), no. 3, 380-393. 3, 53

[18] David Conlon, Jacob Fox, and Yufei Zhao, Extremal results in sparse pseudorandom graphs, Adv. Math. 256 (2014), 206-290. 5

[19] Domingos Dellamonica, Yoshiharu Kohayakawa, Martin Marciniszyn, and Angelika Steger, On the resilience of long cycles in random graphs, Electron. J. Combin. 15 (2008), R32. 5

[20] Domingos Dellamonica, Jr., Yoshiharu Kohayakawa, Vojtěch Rödl, and Andrzej Ruciński, An improved upper bound on the density of universal random graphs, Random Structures Algorithms 46 (2015), no. 2, 274-299. 3

[21] G. A. Dirac, Some theorems on abstract graphs, Proc. London Math. Soc. 2 (1952), 69-81. 1

[22] Julia Ehrenmüller, Existence and enumeration of spanning structures in sparse graphs and hypergraphs, $\mathrm{PhD}$ thesis, Technische Universität Hamburg, 2016. 42, 45 
The BAndwidTh TheOREM In SPARse GRAPHS

[23] Asaf Ferber, Kyle Luh, and Oanh Nguyen, Embedding large graphs into a random graph, Bull. Lond. Math. Soc. 49 (2017), no. 5, 784-797. 2, 3

[24] Hao Huang, Choongbum Lee, and Benny Sudakov, Bandwidth theorem for random graphs, J. Combin. Theory Ser. B 102 (2012), no. 1, 14-37. 4, 6, 52

[25] Svante Janson, Tomasz Łuczak, and Andrzej Ruciński, Random graphs, Wiley-Interscience, 2011. 11,12

[26] Anders Johansson, Jeff Kahn, and Van Vu, Factors in random graphs, Random Structures Algorithms 33 (2008), no. 1, 1-28. 2

[27] Fiachra Knox and Andrew Treglown, Embedding spanning bipartite graphs of small bandwidth, Combin. Probab. Comput. 22 (2013), no. 1, 71-96. 2

[28] Yoshiharu Kohayakawa, Szemerédi's regularity lemma for sparse graphs, Foundations of computational mathematics, Springer, 1997, pp. 216-230. 8, 55

[29] Yoshiharu Kohayakawa and Vojtech Rödl, Szemerédi's regularity lemma and quasi-randomness, Recent advances in algorithms and combinatorics, Springer, 2003, pp. 289-351. 8

[30] János Komlós, Gábor N Sárközy, and Endre Szemerédi, Blow-up lemma, Combinatorica 17 (1997), no. $1,109-123.6,10$

[31] Michael Krivelevich, Choongbum Lee, and Benny Sudakov, Resilient pancyclicity of random and pseudorandom graphs, SIAM J. Discrete Math. 24 (2010), no. 1, 1-16. 5

[32] Daniela Kühn and Deryk Osthus, Embedding large subgraphs into dense graphs, Surveys in combinatorics 2009, London Math. Soc. Lecture Note Ser., vol. 365, Cambridge Univ. Press, Cambridge, 2009, pp. 137-167. 1

[33] C. Lee, Embedding degenerate graphs of small bandwidth, Submitted, arXiv:1501.05350. 2

[34] Choongbum Lee and Benny Sudakov, Dirac's theorem for random graphs, Random Structures Algorithms 41 (2012), no. 3, 293-305. 4

[35] Colin McDiarmid, On the method of bounded differences, Surveys in combinatorics 1989, London Math. Soc. Lecture Note Ser., vol. 141, Cambridge Univ. Press, Cambridge, 1989, pp. 148-188. 13

[36] Rajko Nenadov and Nemanja Škorić, On Komlós' tiling theorem in random graphs, Submitted, arXiv:1611.09466. 4

[37] Andreas Noever and Angelika Steger, Local resilience for squares of almost spanning cycles in sparse random graphs, Electron. J. Combin. 24 (2017), no. 4, P4.8. 4

[38] Oliver Riordan, Spanning subgraphs of random graphs, Combin. Probab. Comput. 9 (2000), no. 2, 125-148. 2, 3 
[39] Mathias Schacht, Extremal results for random discrete structures, Ann. of Math. (2) 184 (2016), no. $2,333-365.2$

[40] Alexander Scott, Szemerédi's regularity lemma for matrices and sparse graphs, Combin. Probab. Comput. 20 (2011), no. 3, 455-466. 27, 54

[41] Benny Sudakov and V. H. Vu, Local resilience of graphs, Random Structures Algorithms 33 (2008), no. $4,409-433.4$

[42] Andrew Thomason, Pseudorandom graphs, Random graphs '85 (Poznań, 1985), North-Holland Math. Stud., vol. 144, North-Holland, Amsterdam, 1987, pp. 307-331. 5

\section{AUTHORS}

Peter Allen

London School of Economics,

Department of Mathematics,

Houghton Street,

London WC2A 2AE, UK

p.d.allen@1se.ac.uk

Julia Böttcher

London School of Economics,

Department of Mathematics,

Houghton Street,

London WC2A 2AE, UK

j.boettcher@1se.ac.uk

Julia Ehrenmüller

Technische Universität Hamburg,

Institut für Mathematik,

Am Schwarzenberg-Campus 3,

21073 Hamburg, Germany

julia.ehrenmueller@gmail.com

Anusch Taraz

Technische Universität Hamburg, Institut für Mathematik,

Am Schwarzenberg-Campus 3, 21073 Hamburg, Germany

taraz@tuhh.de 